\title{
The contribution of interactions to
} arthropod community structure depends on the place and time: Why community ecologists should think locally and regionally

Matthew K. Lau, Laura E. Hagenauer and Thomas G.Whitham

\author{
Department of Biological Sciences \\ Northern Arizona University
}



- Disintegrating the community (Ricklefs 2008) 
- Disintegrating the community (Ricklefs 2008)

- Brooks et al. 2009 
- Disintegrating the community (Ricklefs 2008)

- Brooks et al. 2009

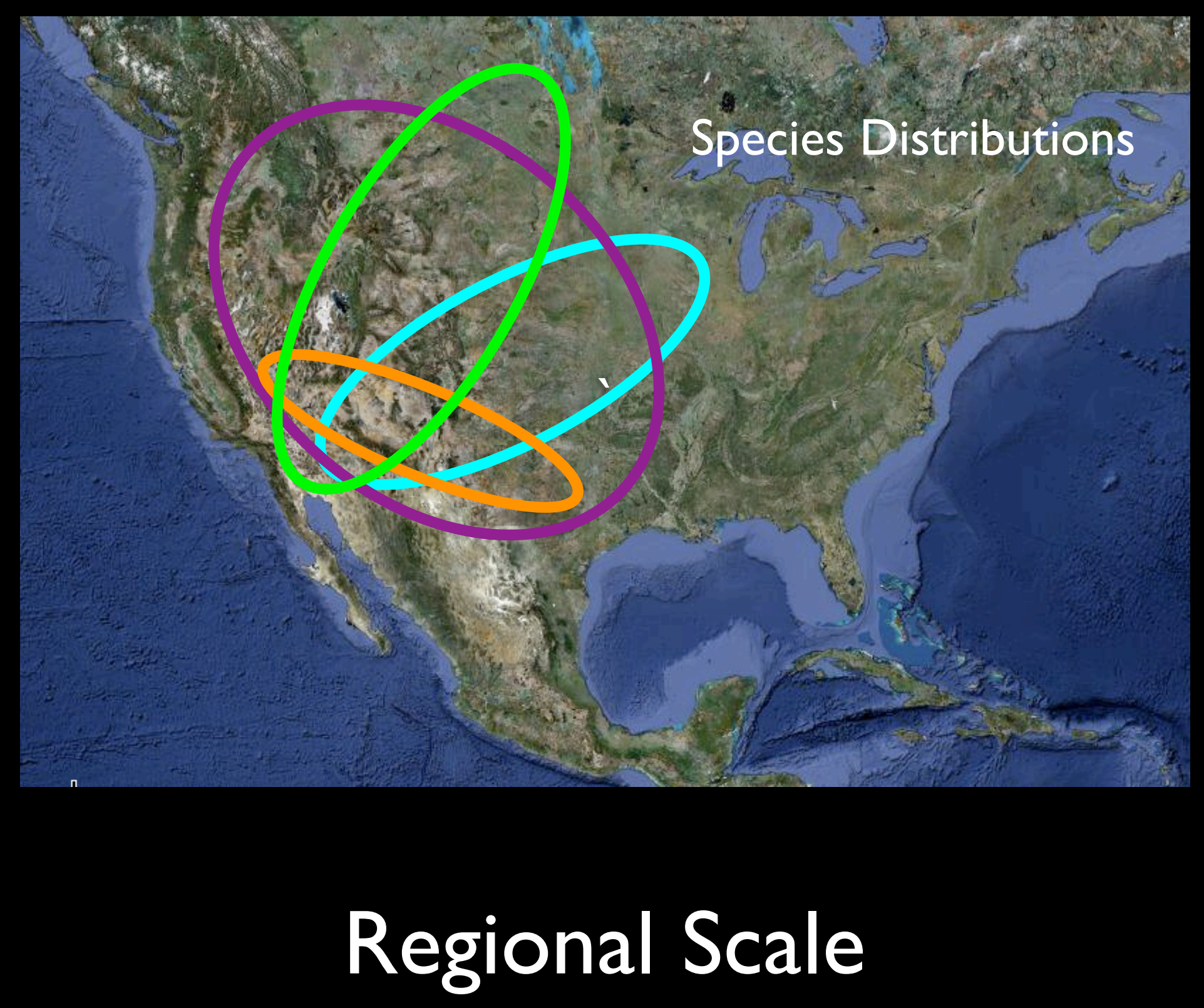


- Disintegrating the community (Ricklefs 2008)

- Brooks et al. 2009
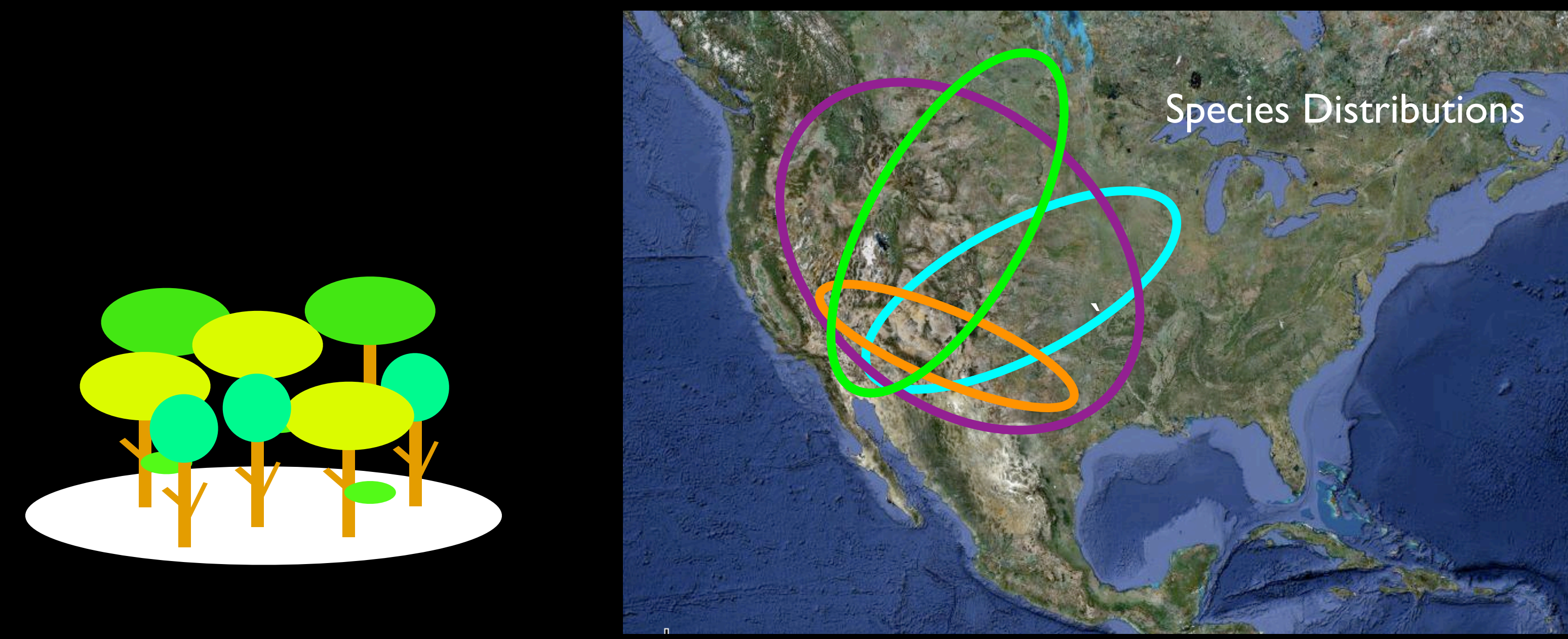

Local Scale

\section{Regional Scale}


- Disintegrating the community (Ricklefs 2008)

- Brooks et al. 2009

- Local community structure can be affected by local interactions, context dependent 
- Disintegrating the community (Ricklefs 2008)

- Brooks et al. 2009

- Local community structure can be affected by local interactions, context dependent

- What does this mean for the study of communities? 


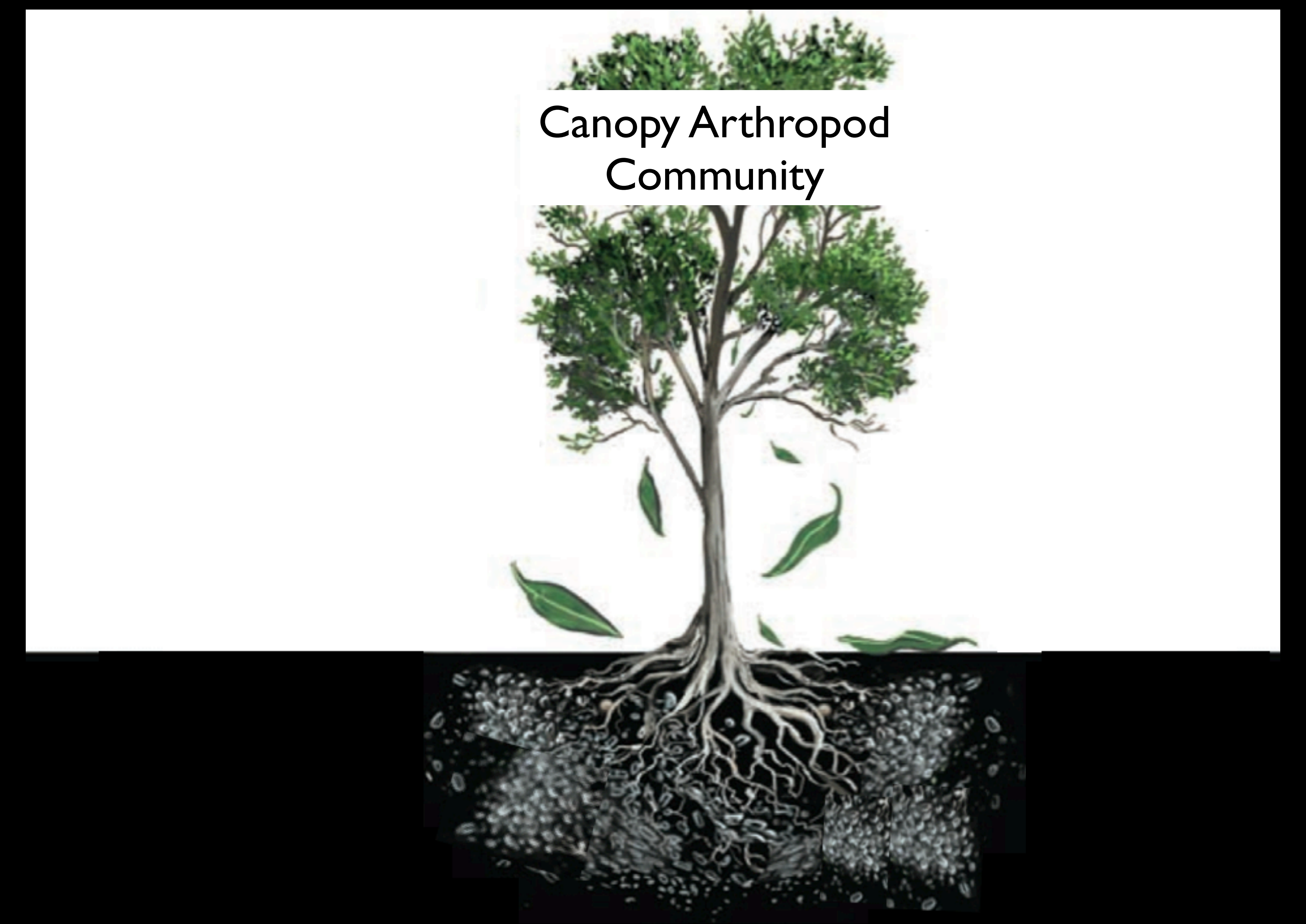




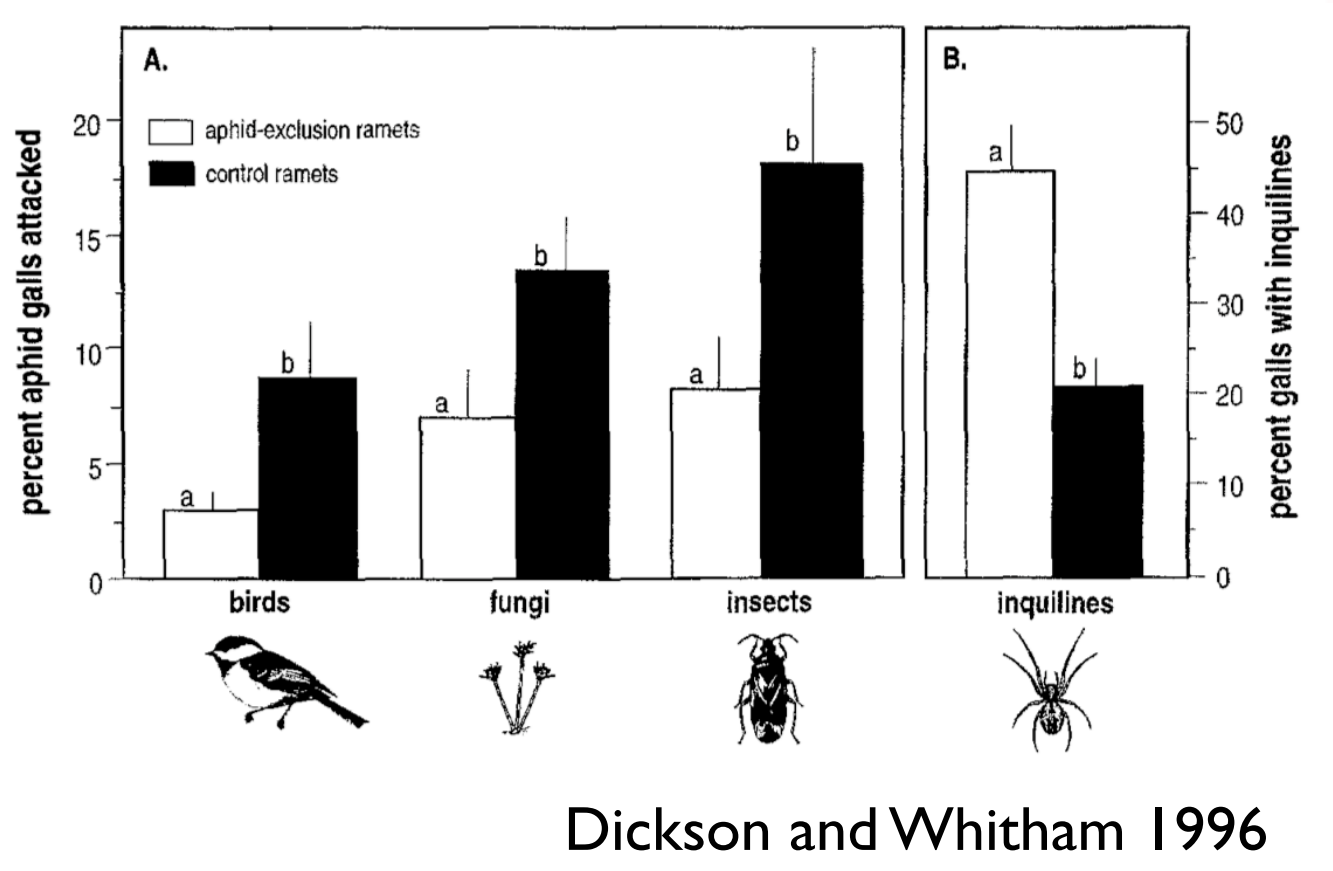

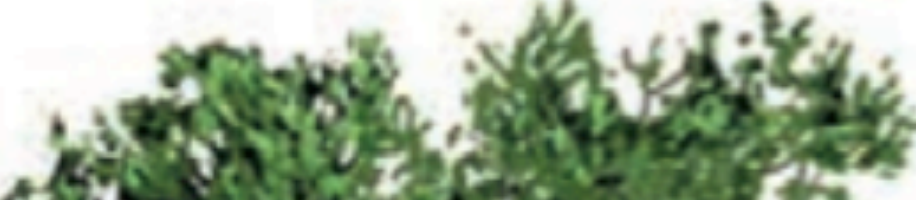

Canopy Arthropod Community
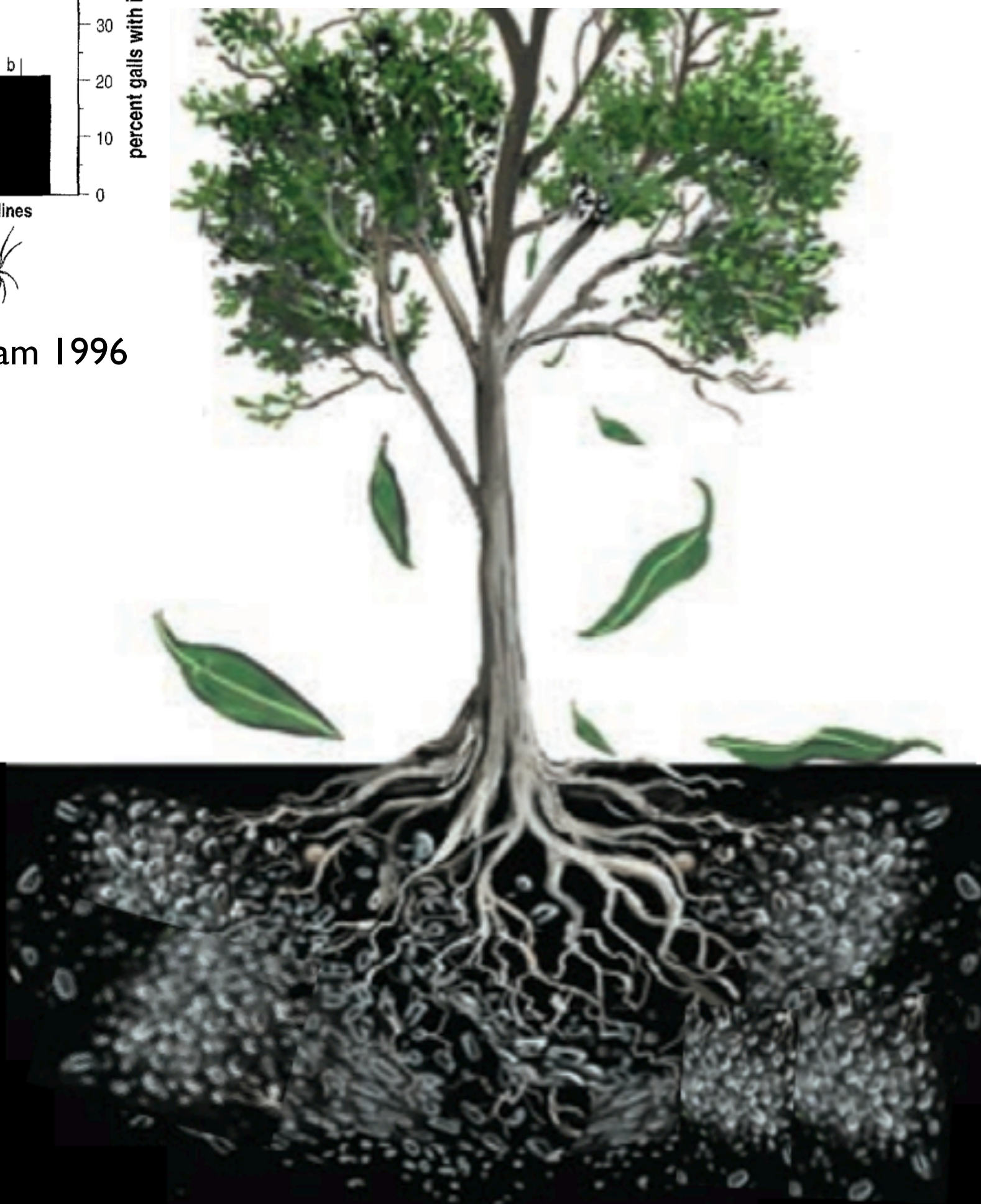


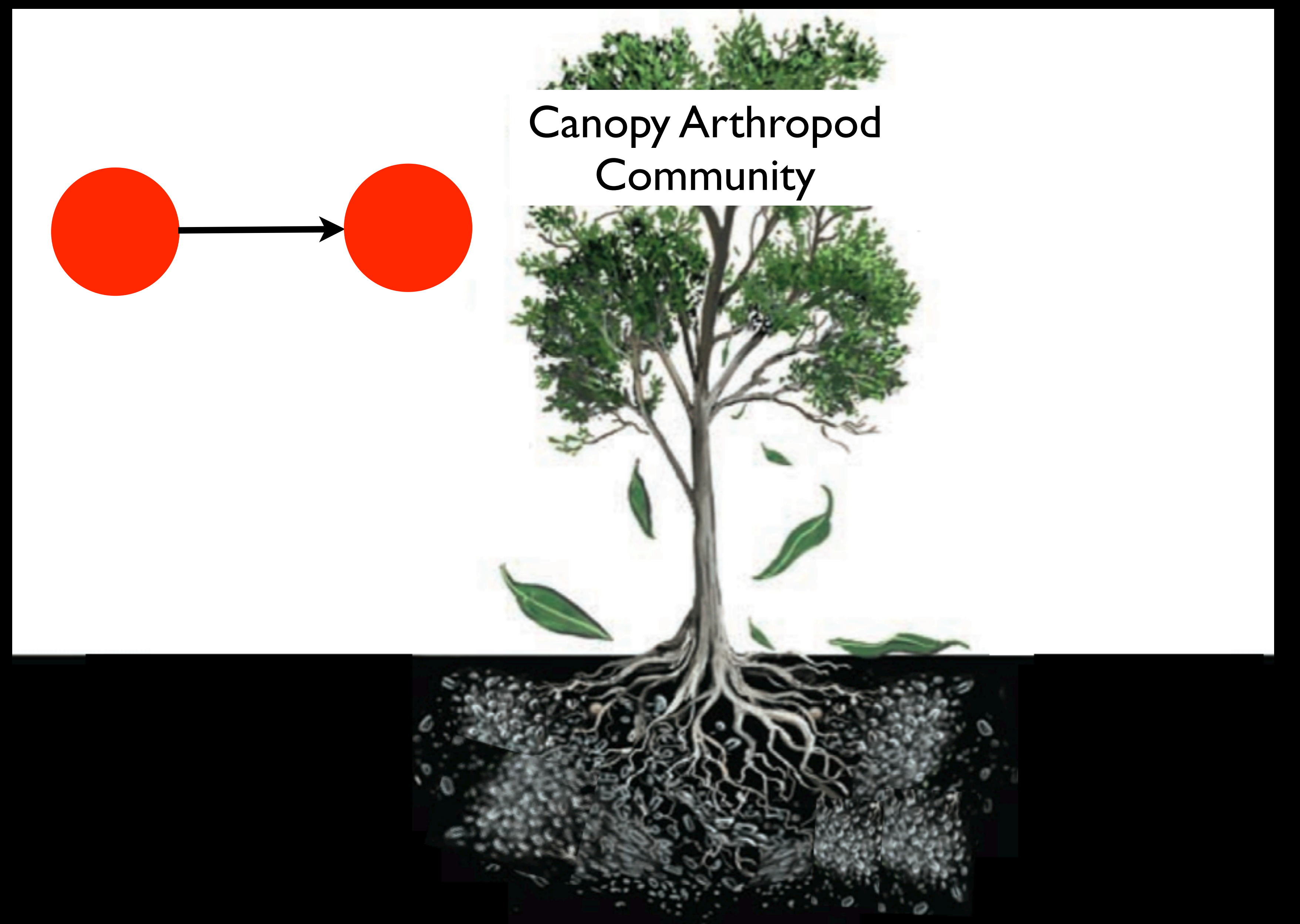




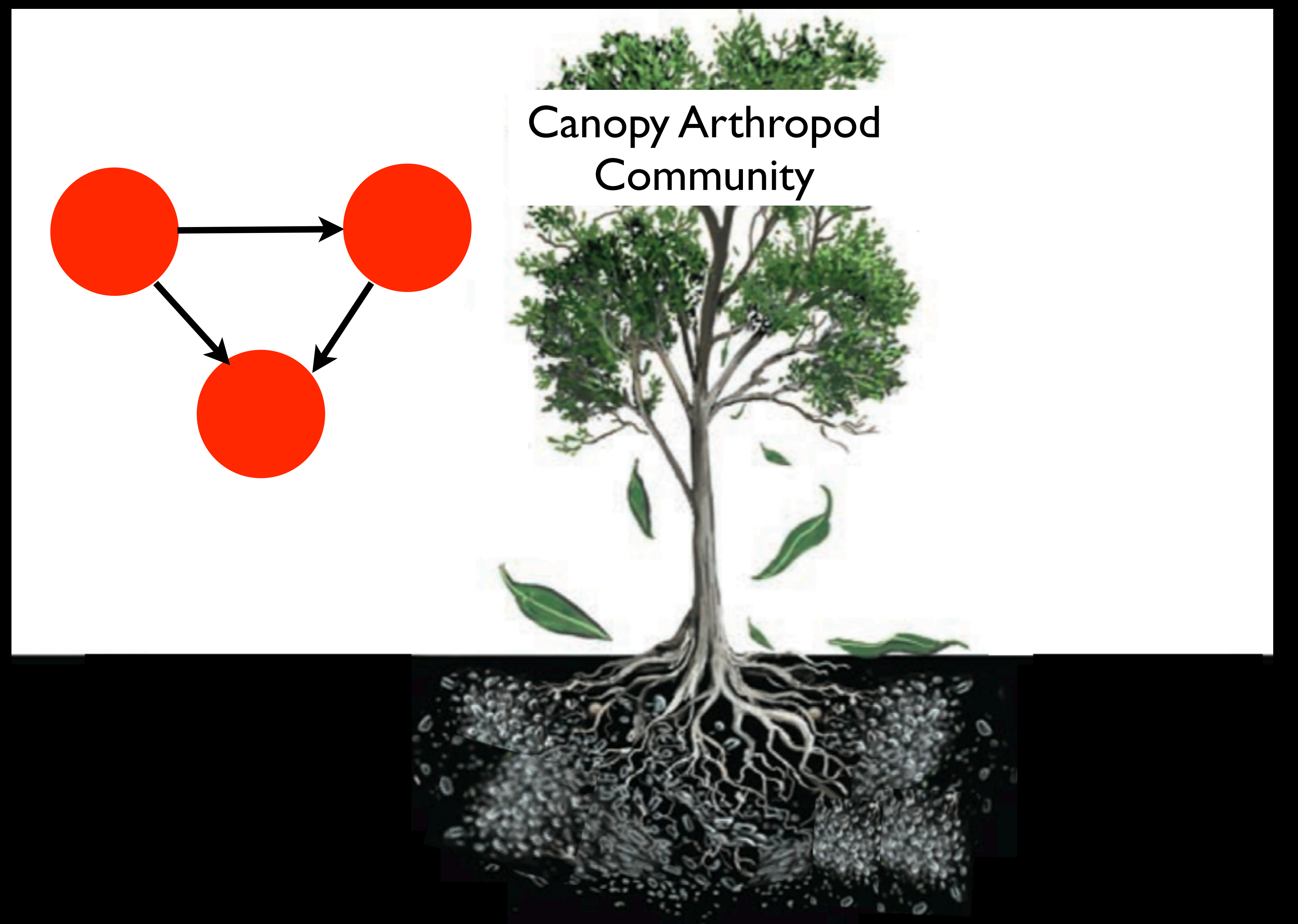




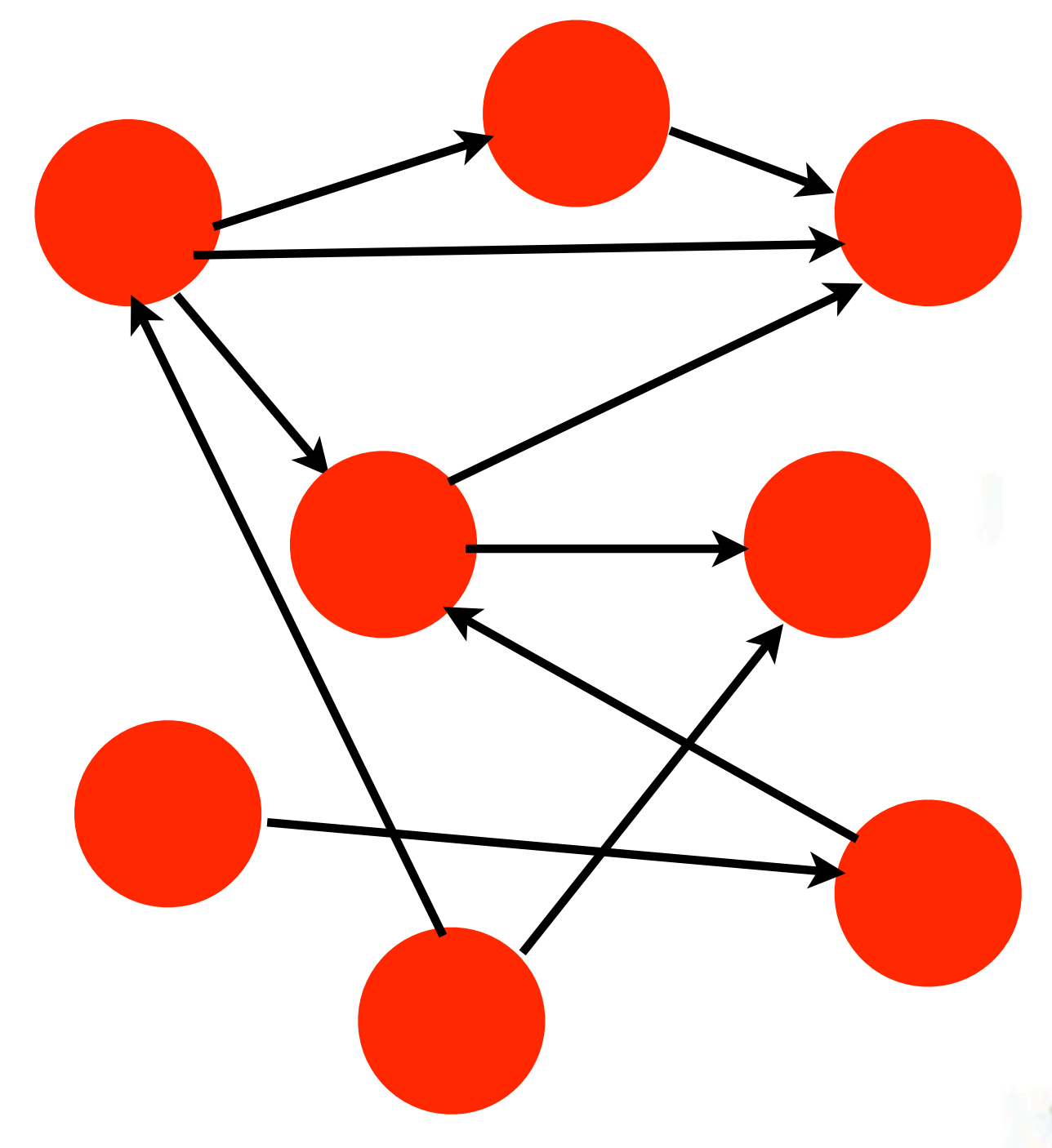

Nond

Canopy Arthropod Community 3.

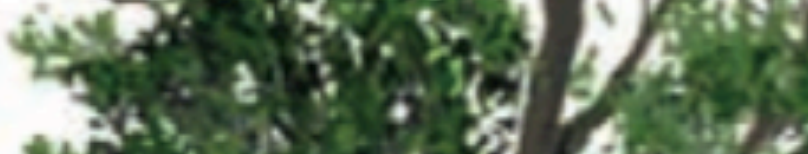
* * *

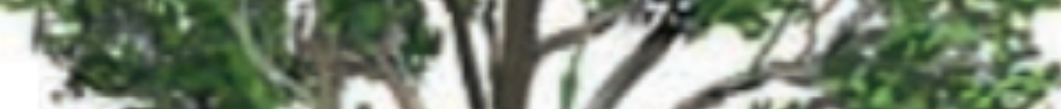
$2 x+\sqrt{1}+6 \%$ 1.

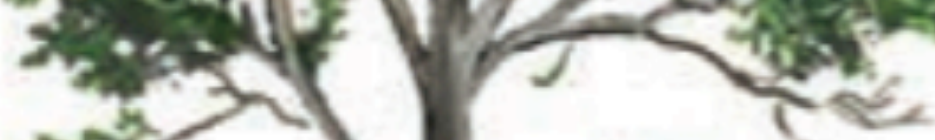
.

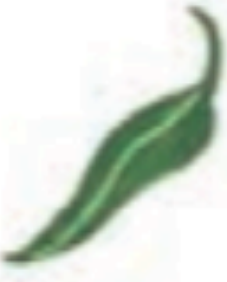

\&ision $00 / 1$

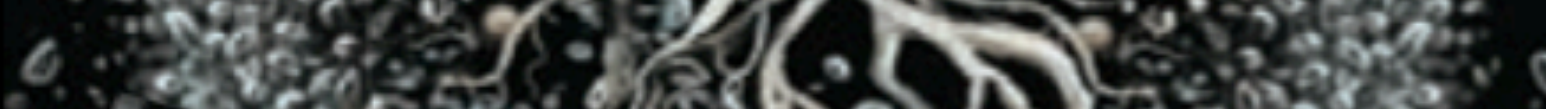

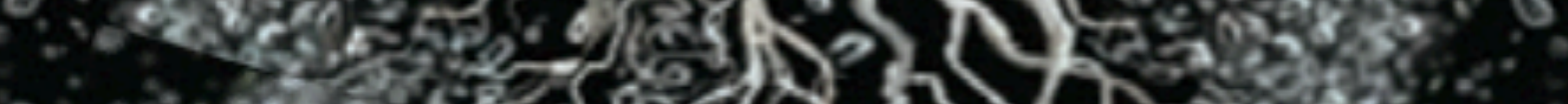

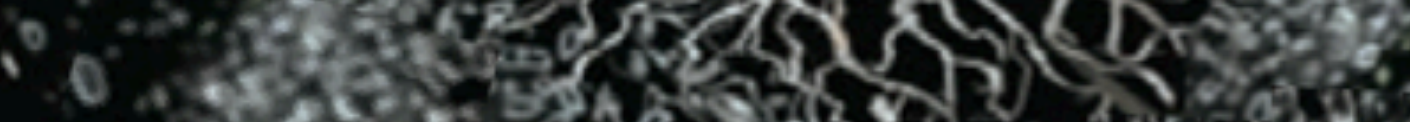

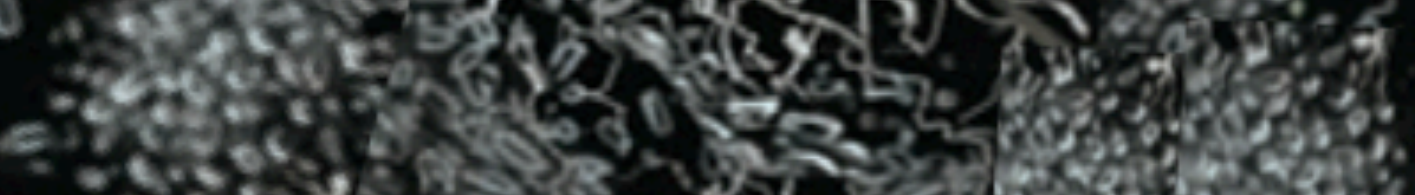

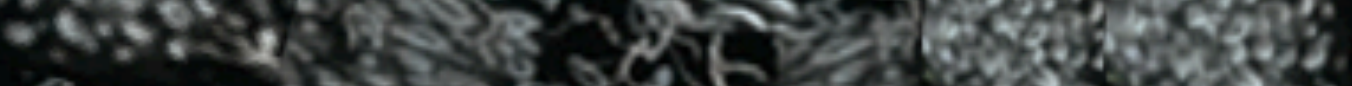

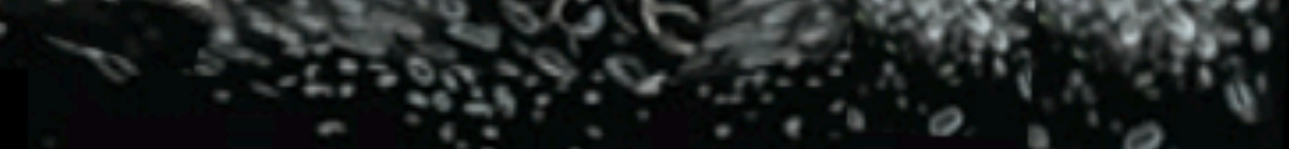


Canopy Arthropod Community

\section{How important are} interactions of canopy arthropod species? 


\section{Study System}

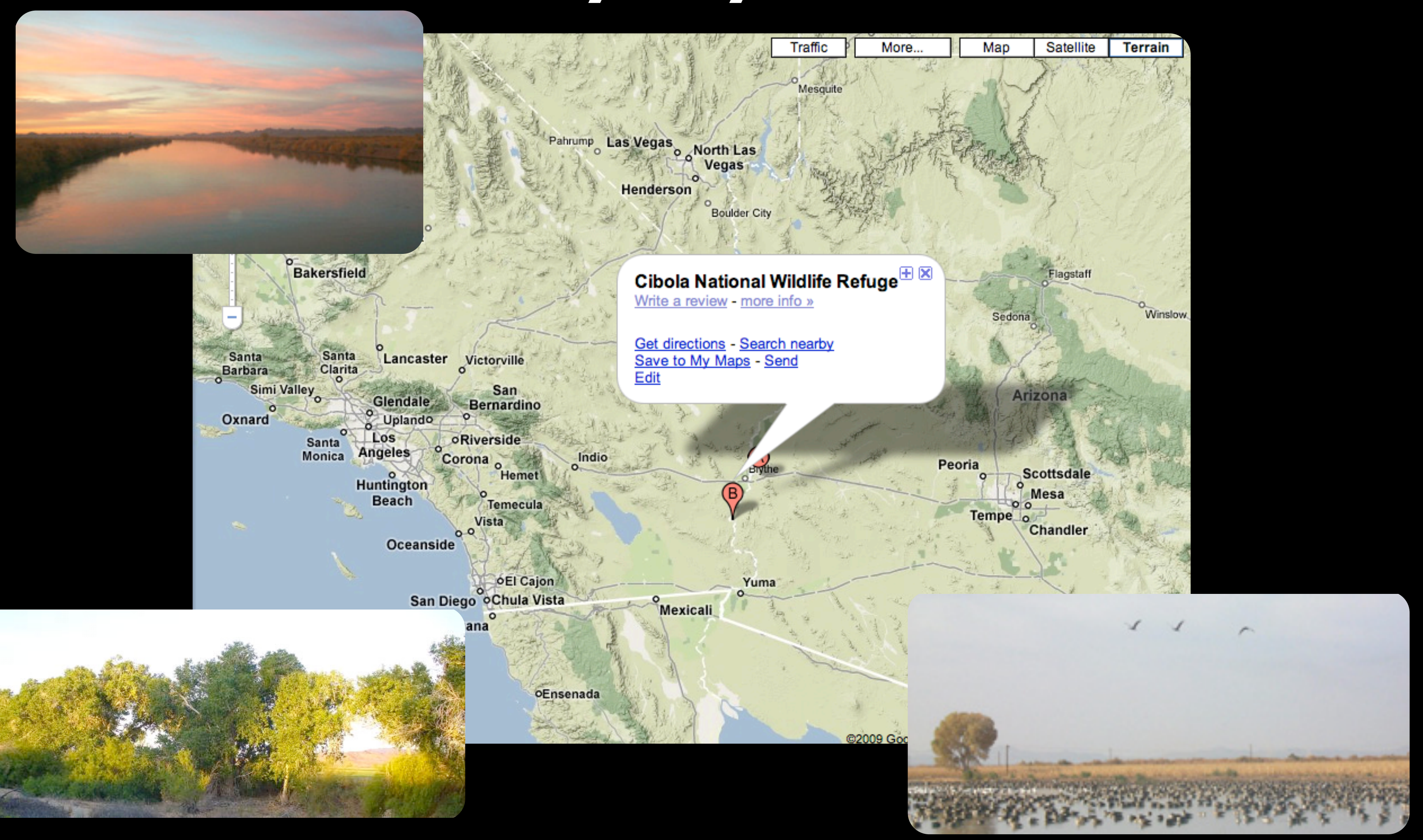




\section{Study System}

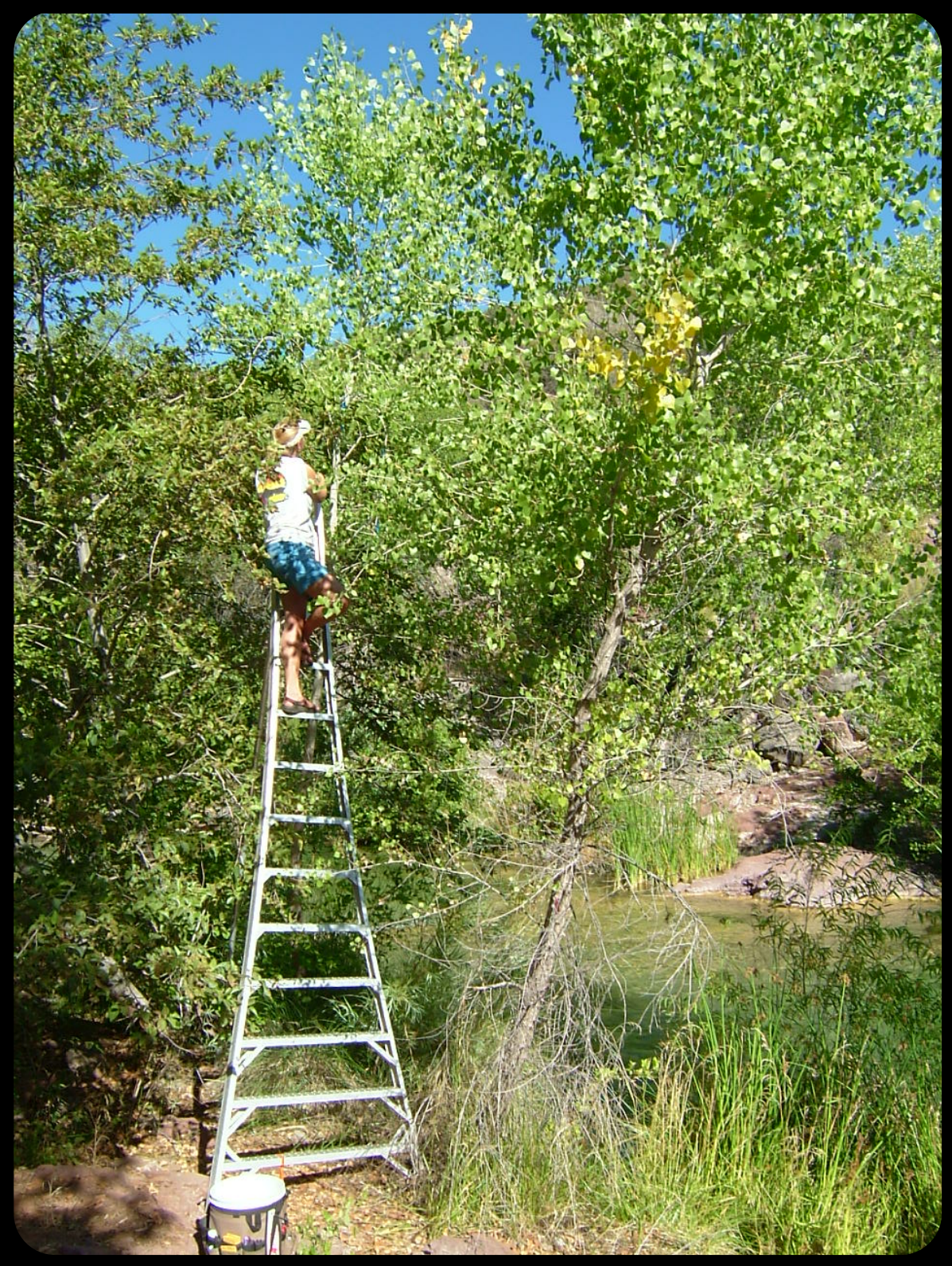




\section{Study System}

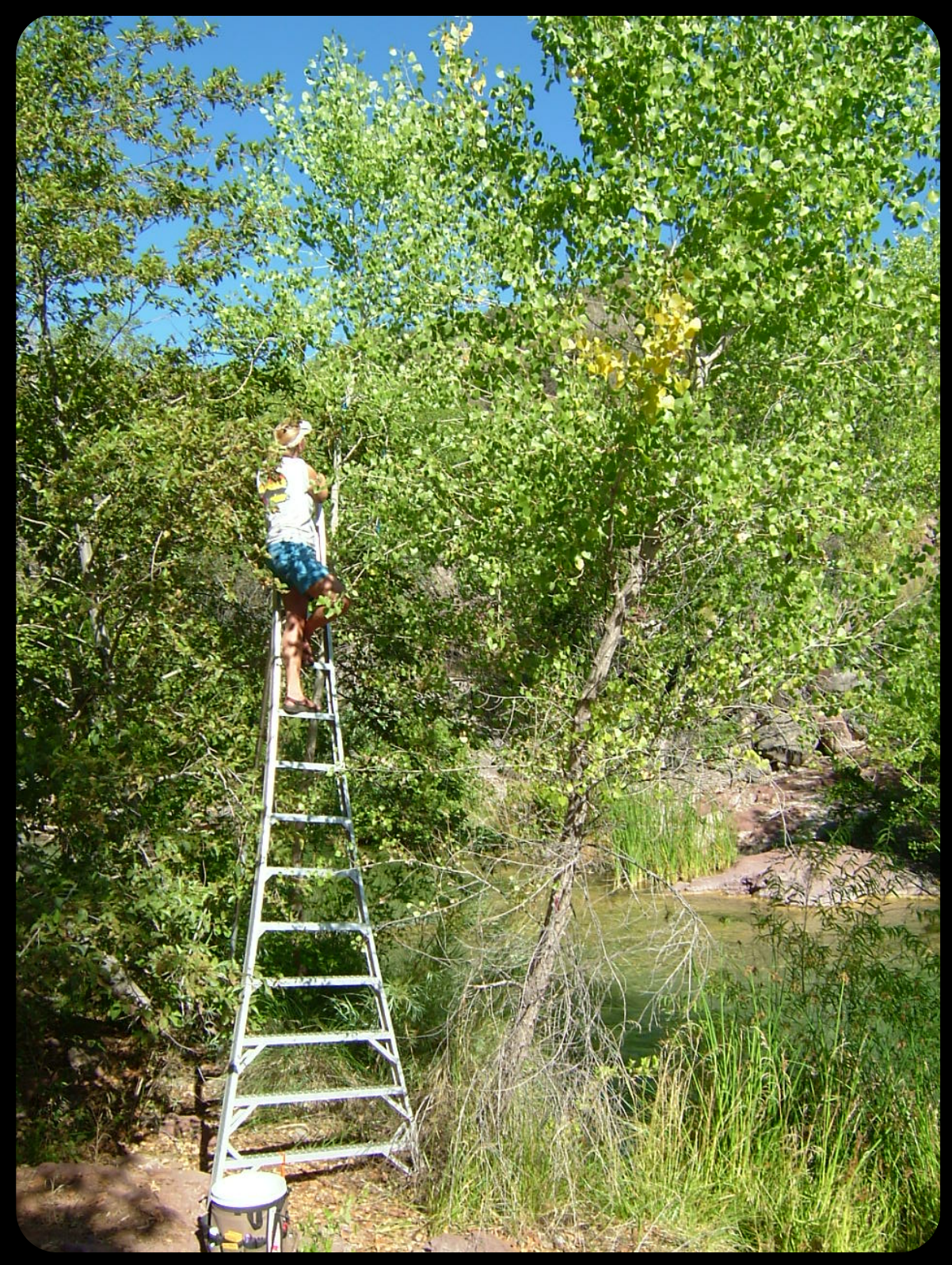

- Three stands of cottonwood 


\section{Study System}

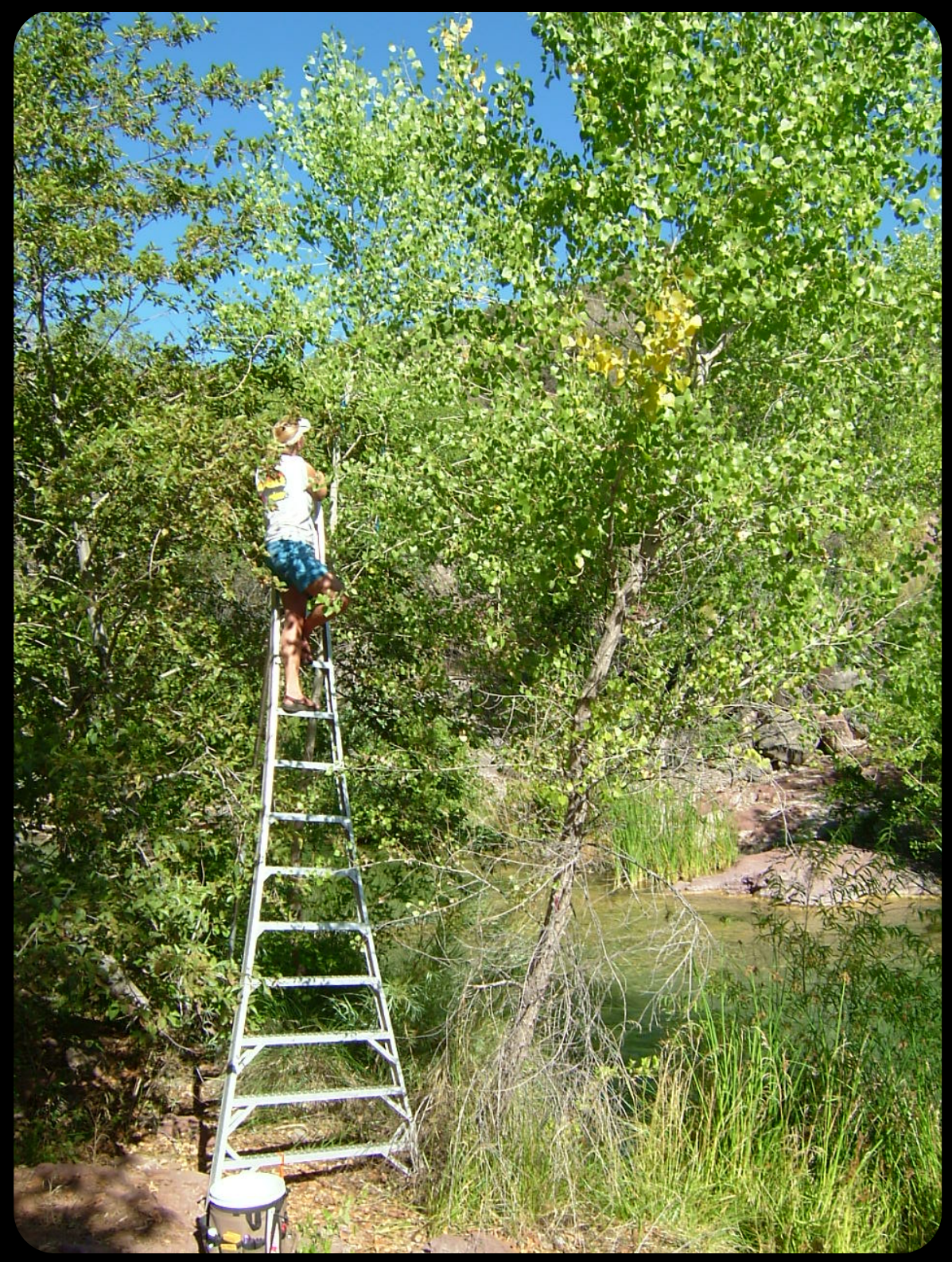

- Three stands of cottonwood - Populus fremontii 


\section{Study System}

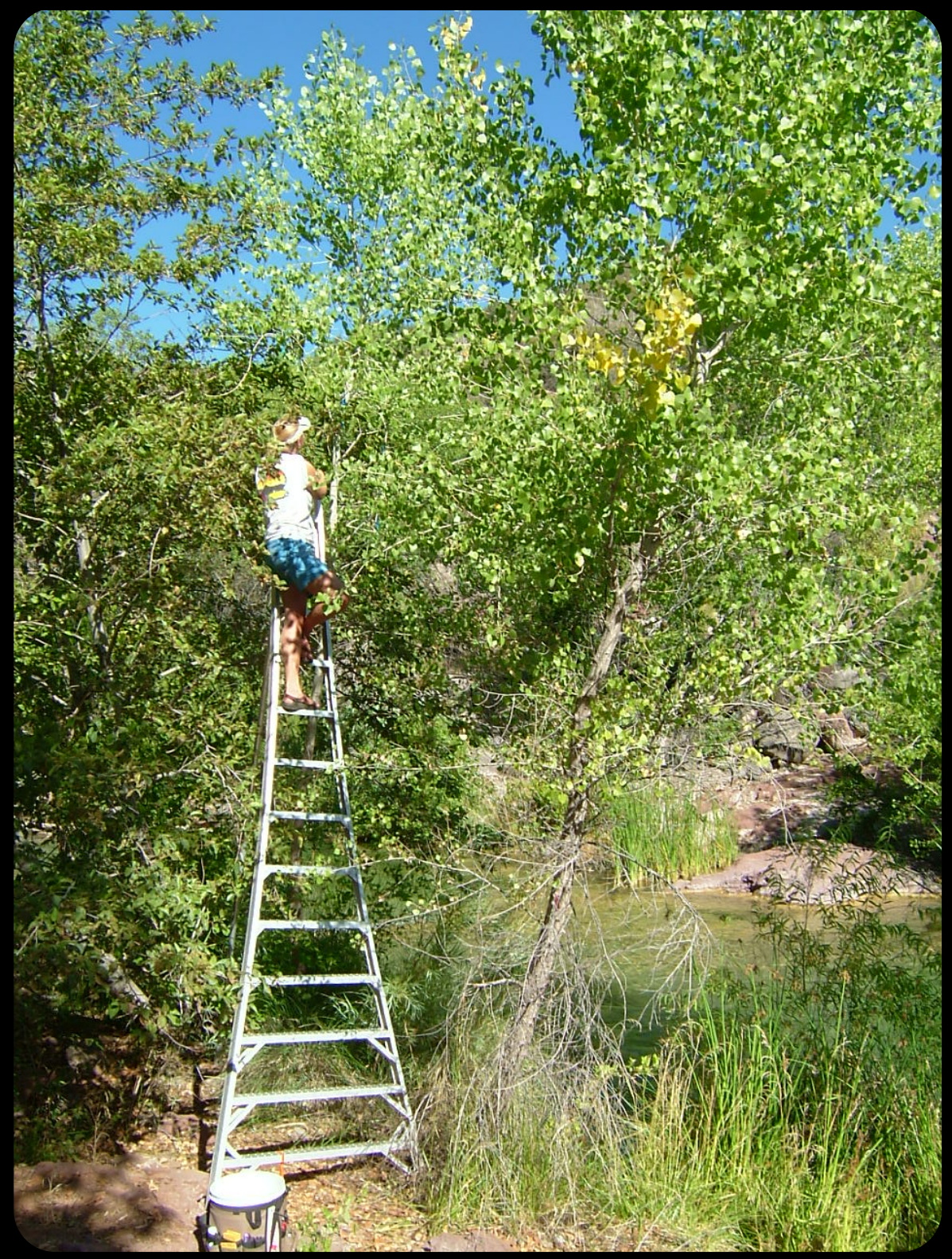

- Three stands of cottonwood

- Populus fremontii

- Planting dates $(2000,2002,2005)$ 


\section{Study System}

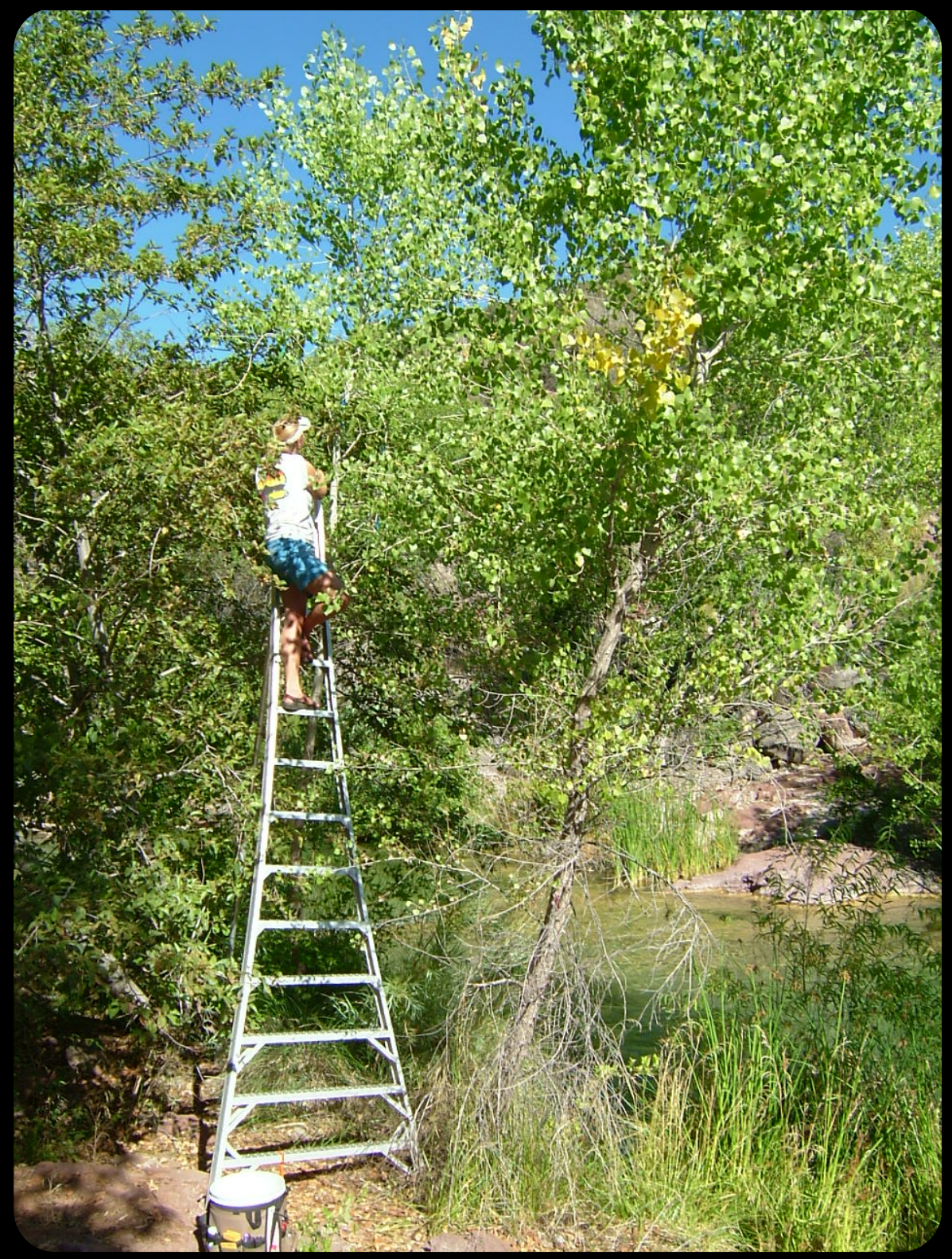

- Three stands of cottonwood

- Populus fremontii

- Planting dates $(2000,2002,2005)$

- $\sim 650$ trees 


\section{Study System}

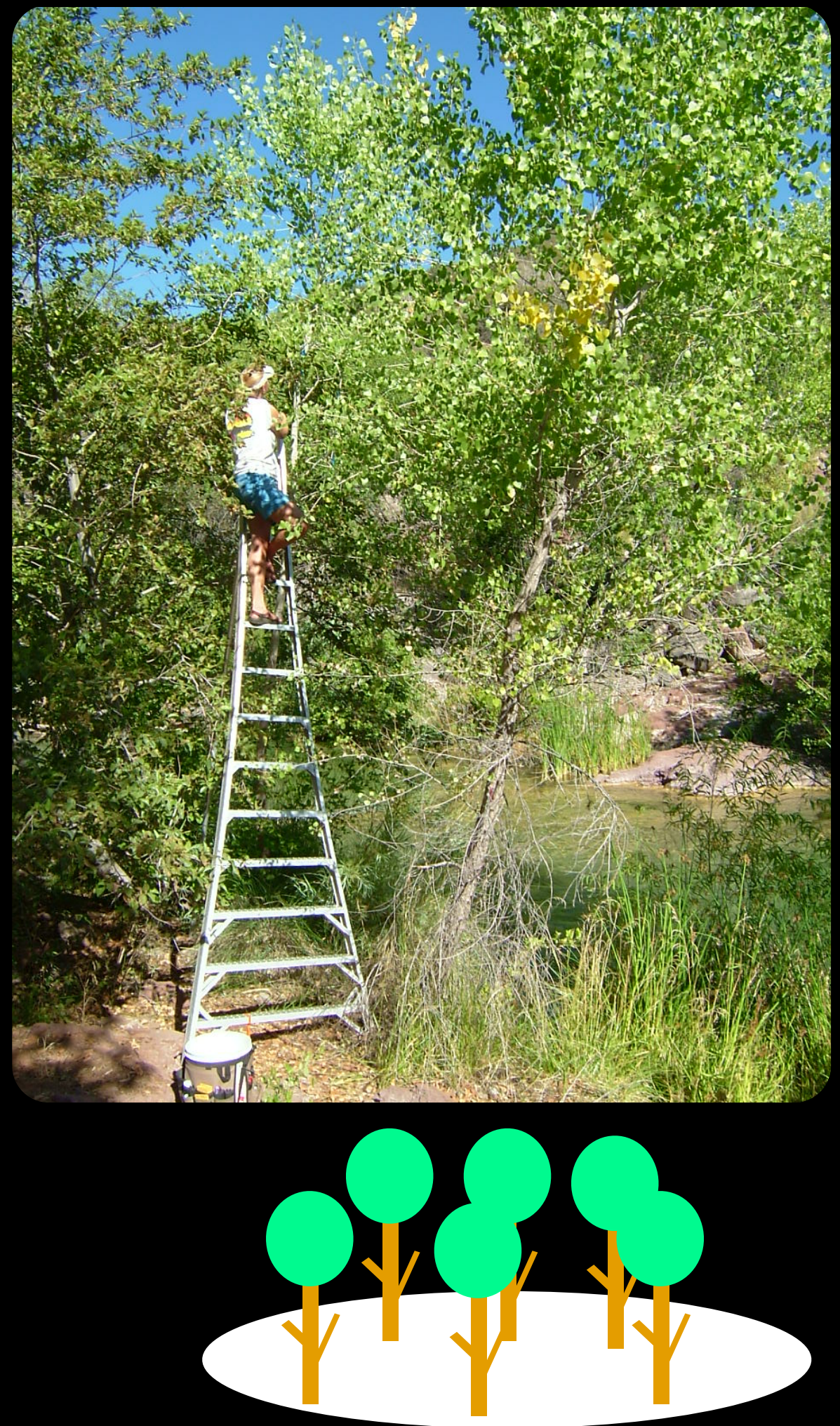

- Three stands of cottonwood

- Populus fremontii

- Planting dates $(2000,2002,2005)$

- $\sim 650$ trees 


\section{Study System}

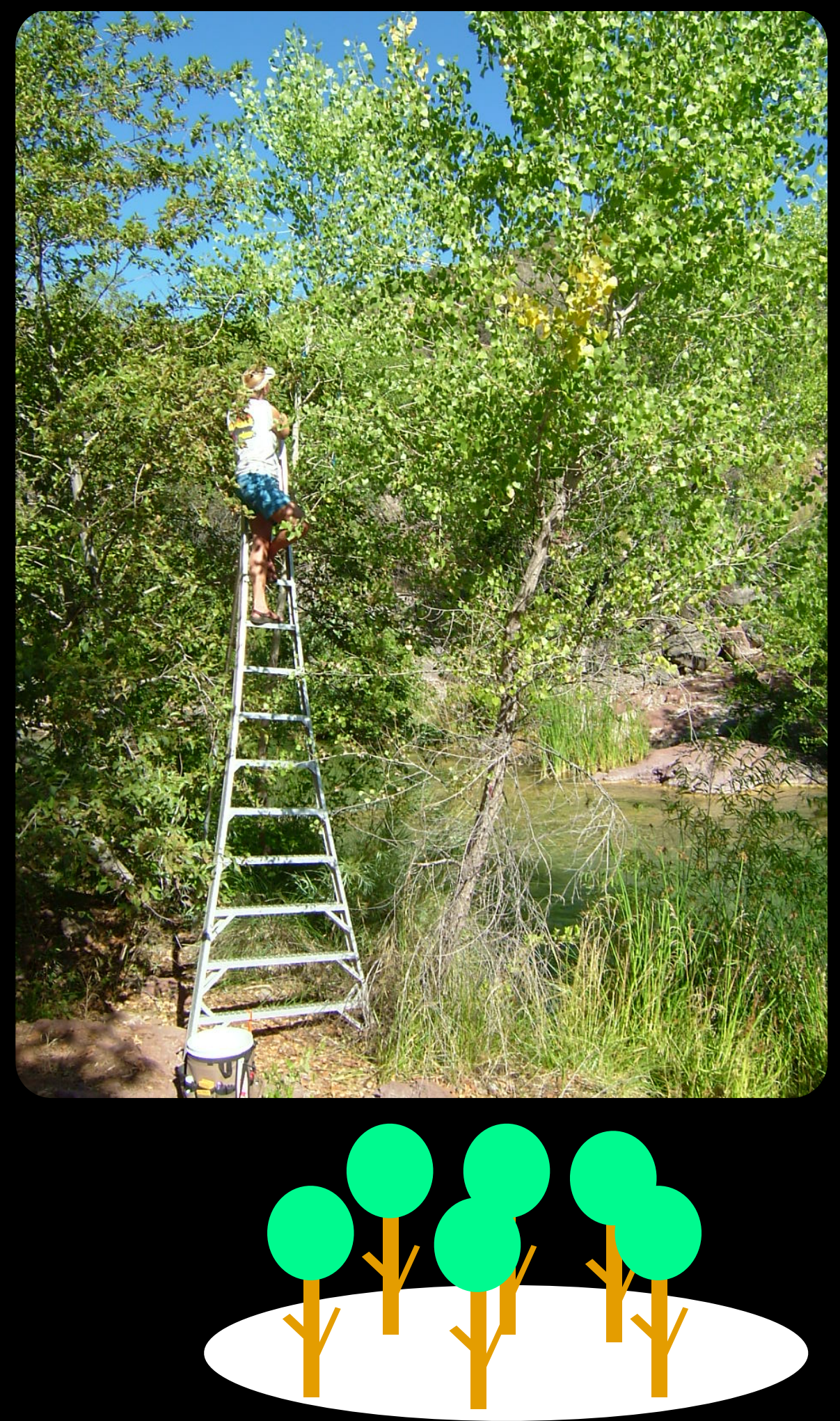

- Three stands of cottonwood

- Populus fremontii

- Planting dates $(2000,2002,2005)$

- $\sim 650$ trees

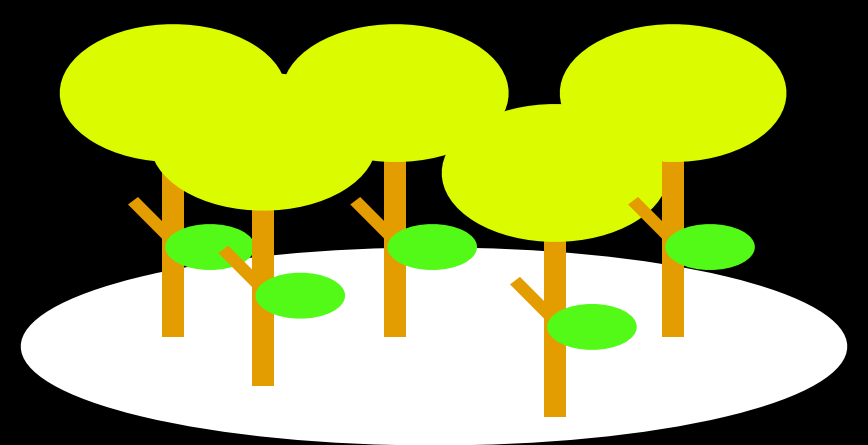




\section{Study System}

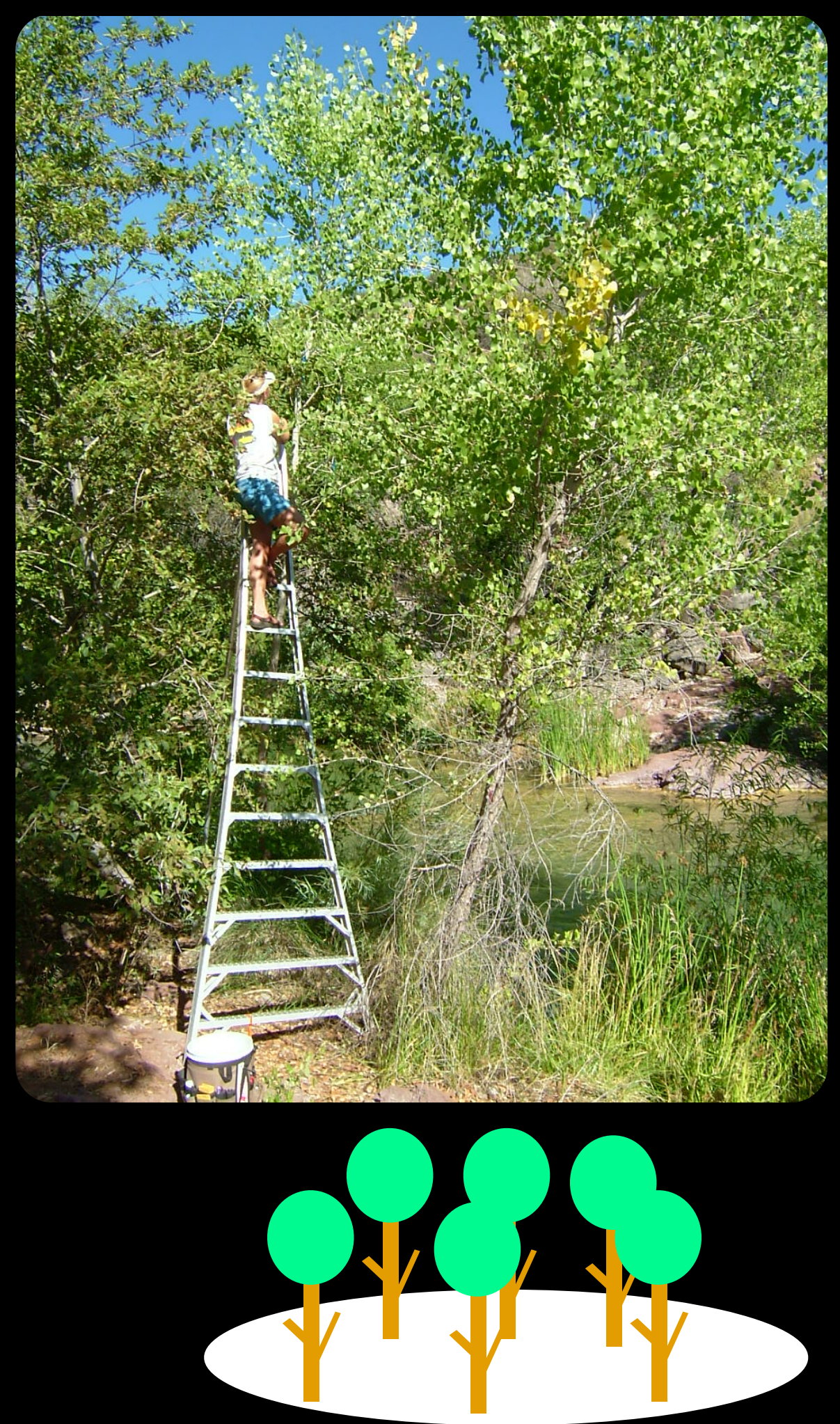

- Three stands of cottonwood

- Populus fremontii

- Planting dates $(2000,2002,2005)$

- $\sim 650$ trees
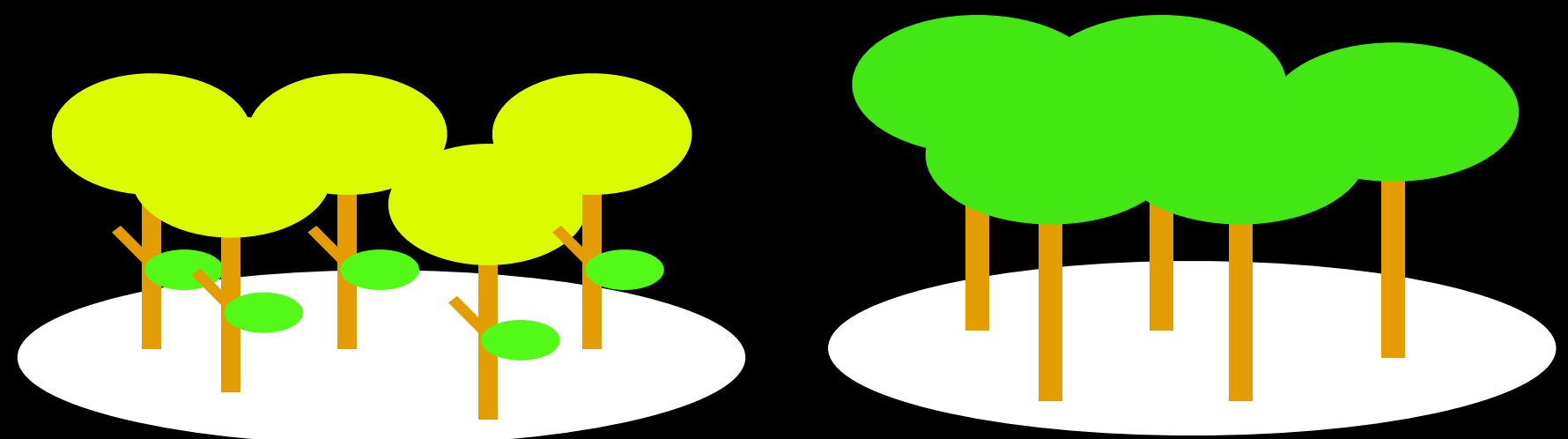


\section{Study System}

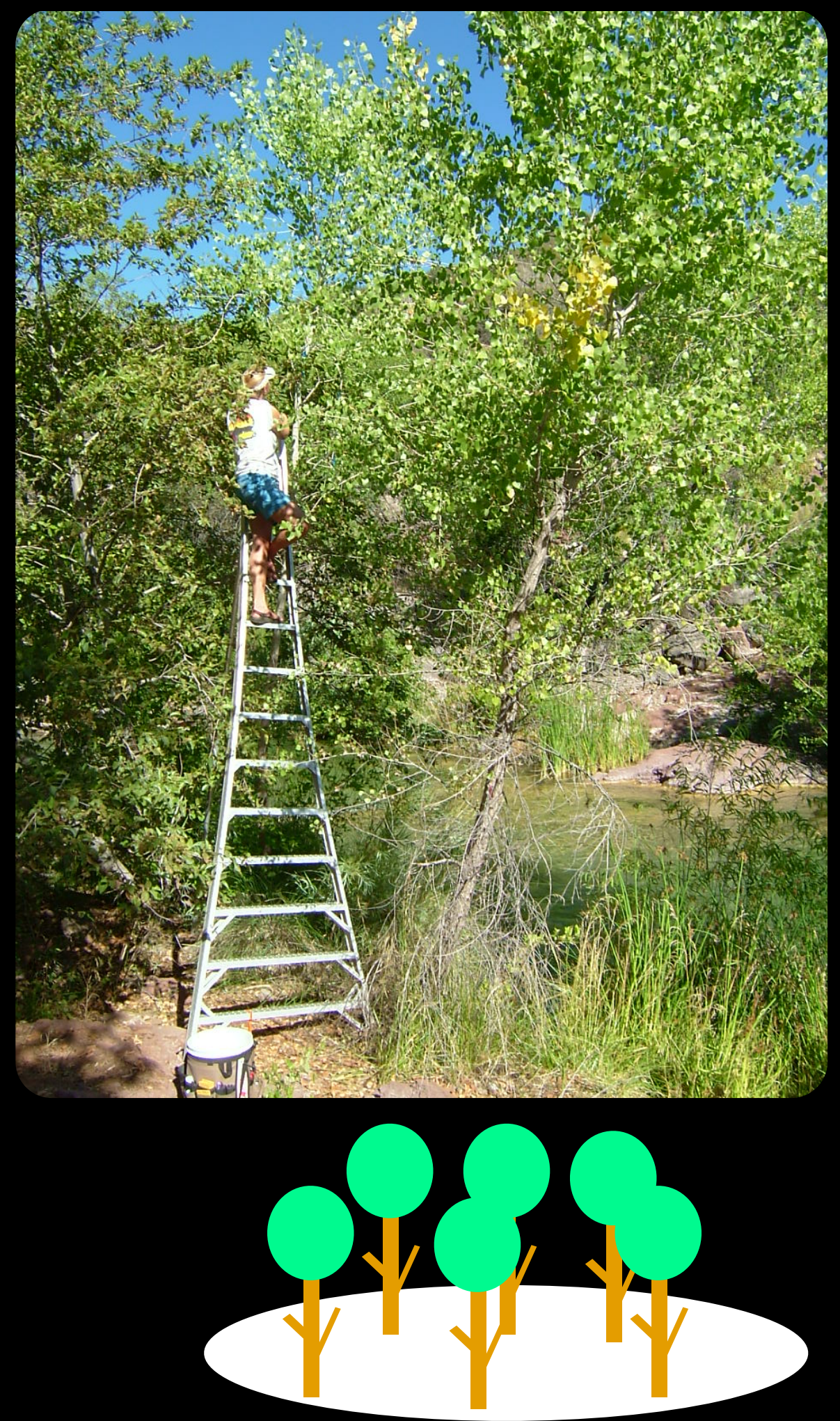

- Three stands of cottonwood

- Populus fremontii

- Planting dates $(2000,2002,2005)$

- $\sim 50$ trees

- Sampling $(n=15)$
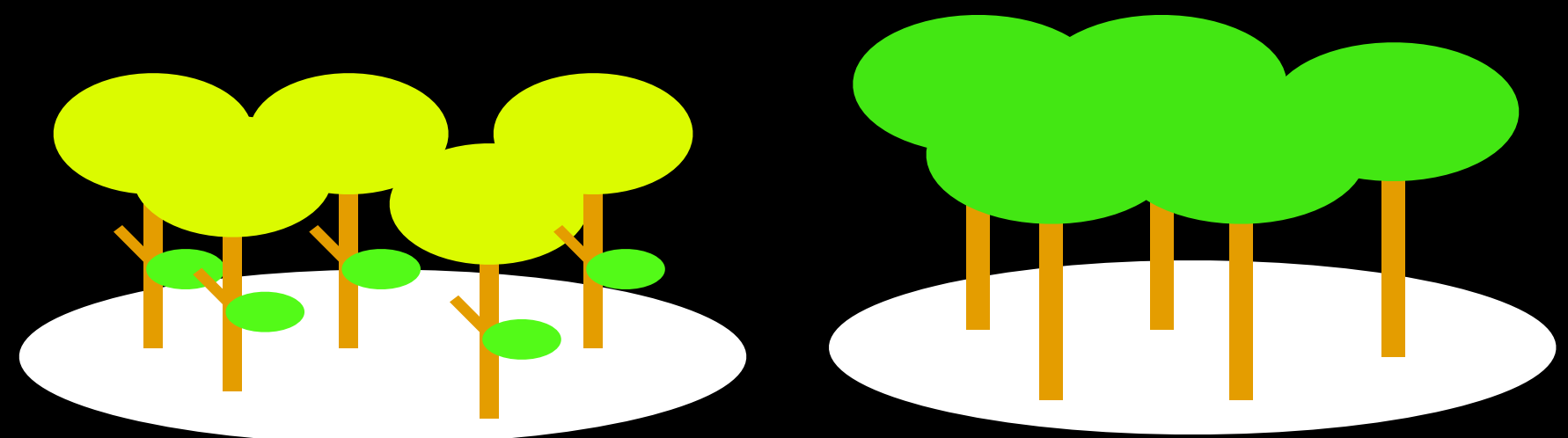


\section{Study System}

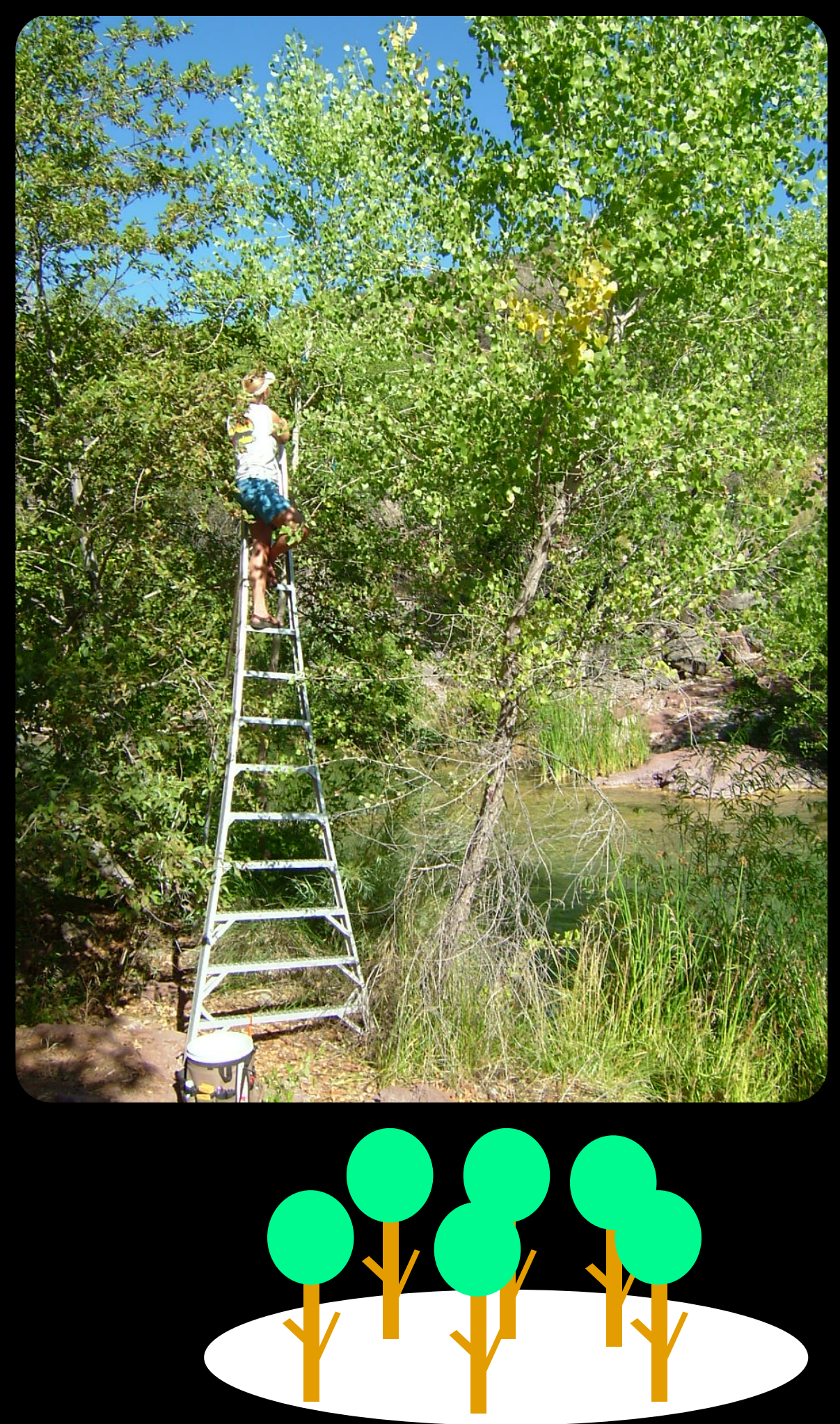

- Three stands of cottonwood

- Populus fremontii

- Planting dates $(2000,2002,2005)$

- 650 trees

- Sampling $(n=15)$

- Sampled monthly in 2006
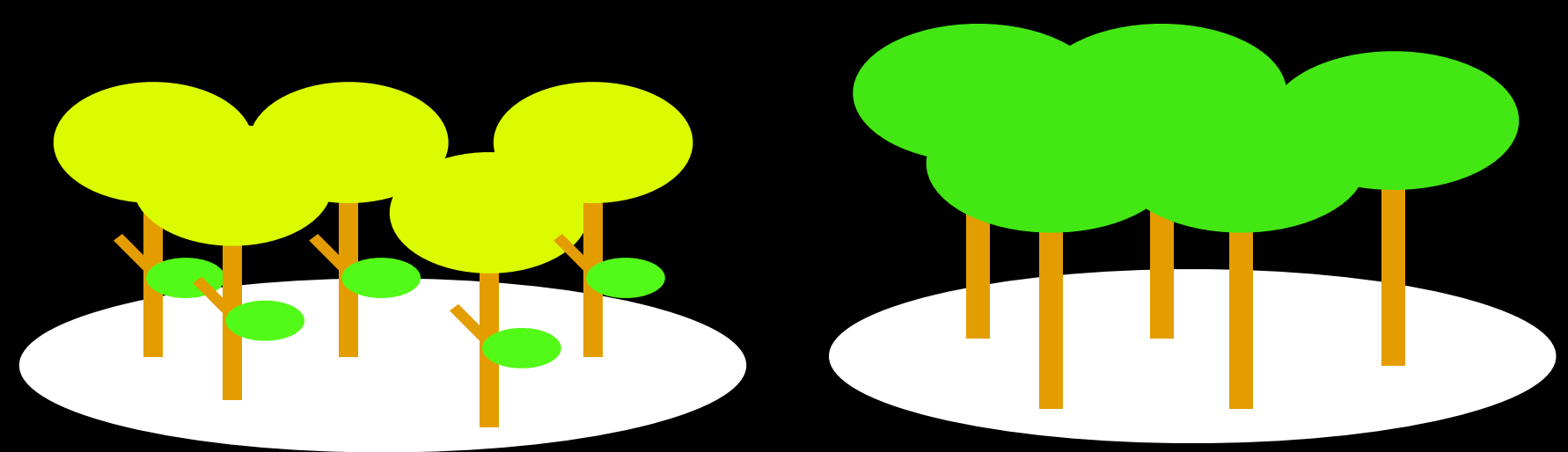


\section{Study System}

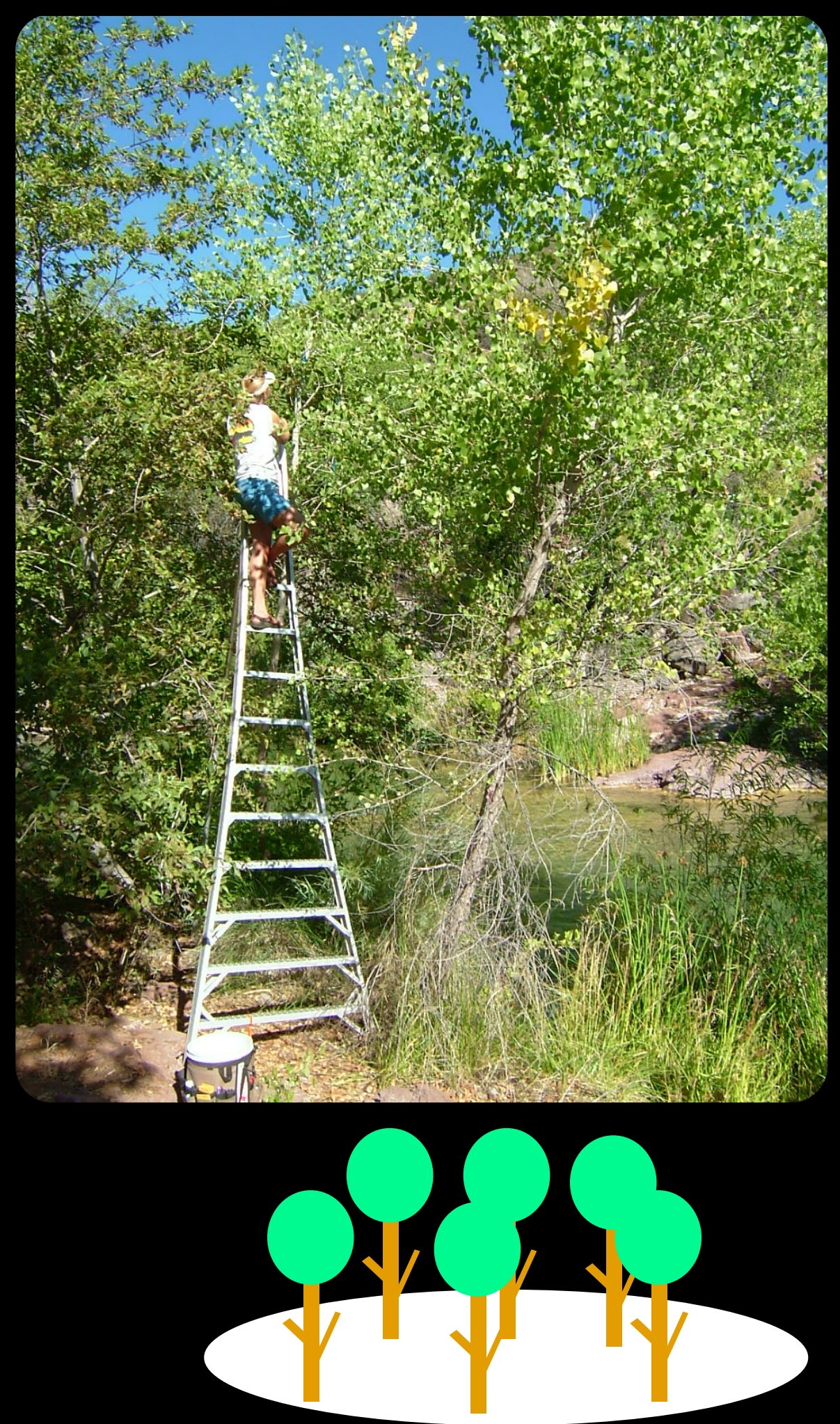

- Three stands of cottonwood

- Populus fremontii

- Planting dates $(2000,2002,2005)$

- 650 trees

- Sampling $(n=15)$

- Sampled monthly in 2006

- Timed-observation sampling
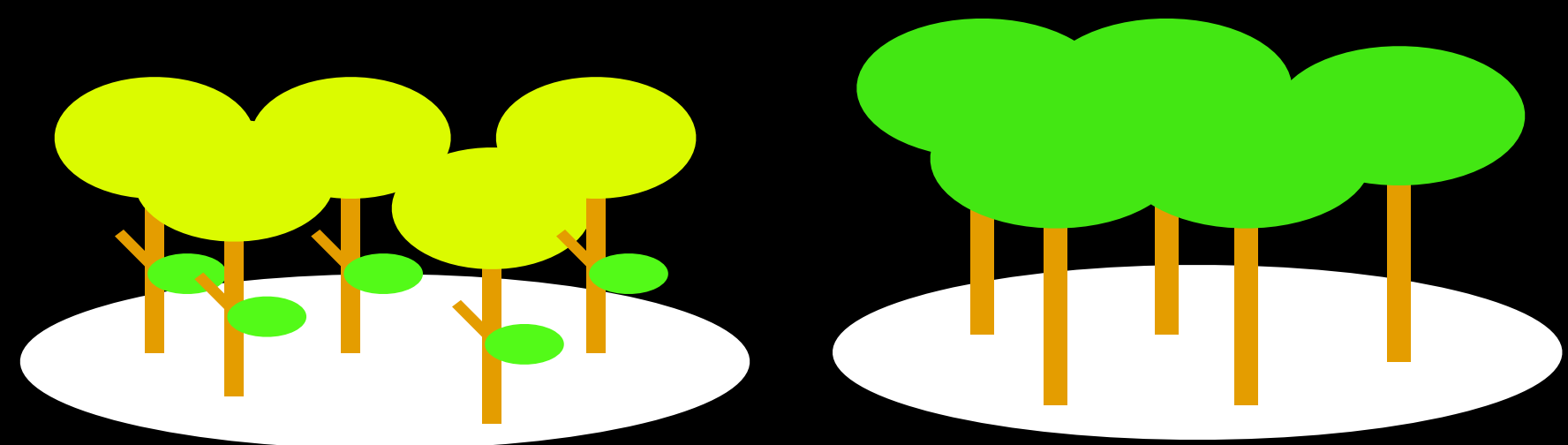


\section{Study System}

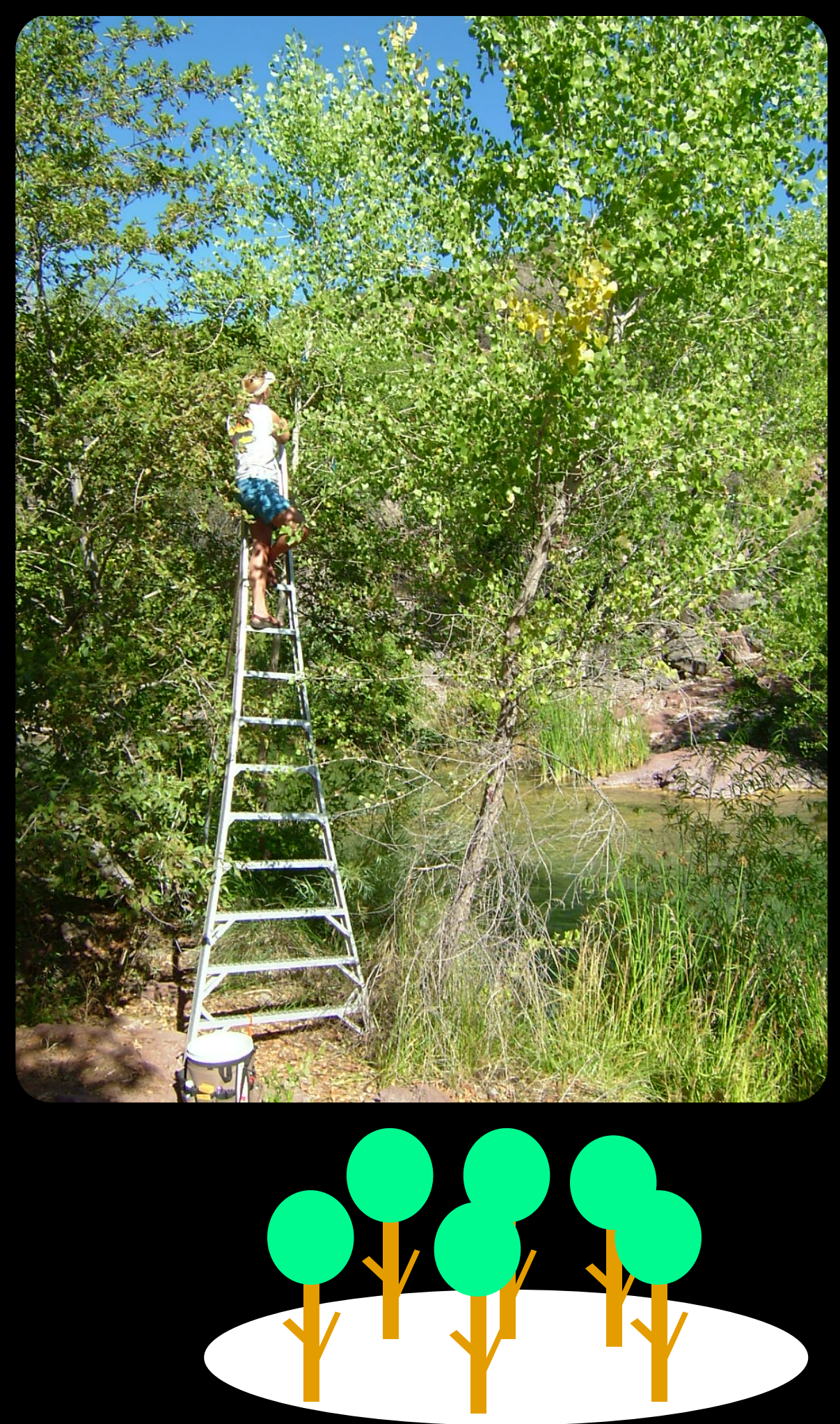

- Three stands of cottonwood

- Populus fremontii

- Planting dates $(2000,2002,2005)$

- 650 trees

- Sampling ( $\mathrm{n}=15$ )

- Sampled monthly in 2006

- Timed-observation sampling

- Juvenile foliage
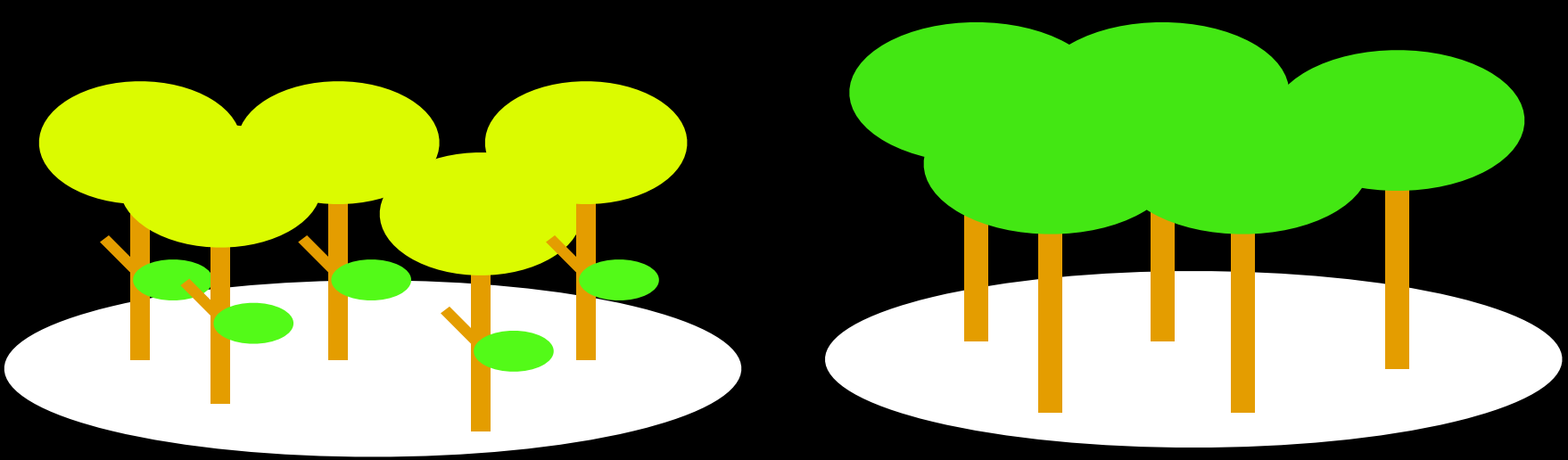


\section{Co-occurrence Analysis}




\section{Co-occurrence Analysis}

Occurrence Matrix

\begin{tabular}{|c|c|c|c|}
\hline Species & ObsI & Obs2 & Obs3 \\
\hline SpI & $\mathrm{I}$ & $\mathrm{I}$ & 0 \\
\hline Sp2 & 0 & 0 & $\mathrm{I}$ \\
\hline Sp3 & 0 & $\mathrm{I}$ & $\mathrm{I}$ \\
\hline
\end{tabular}




\section{Co-occurrence Analysis}

Occurrence Matrix

\begin{tabular}{|c|c|c|c|}
\hline Species & Obs I & Obs2 & Obs3 \\
\hline SpI & 1 & 1 & 0 \\
\hline Sp2 & 0 & 0 & 1 \\
\hline Sp3 & 0 & 1 & 1 \\
\hline
\end{tabular}




\section{Co-occurrence Analysis}

Occurrence Matrix

\begin{tabular}{|c|c|c|c|}
\hline Species & ObsI & Obs2 & Obs3 \\
\hline SpI & $\mathrm{I}$ & $\mathrm{I}$ & 0 \\
\hline Sp2 & 0 & 0 & $\mathrm{I}$ \\
\hline Sp3 & 0 & $\mathrm{I}$ & $\mathrm{I}$ \\
\hline
\end{tabular}

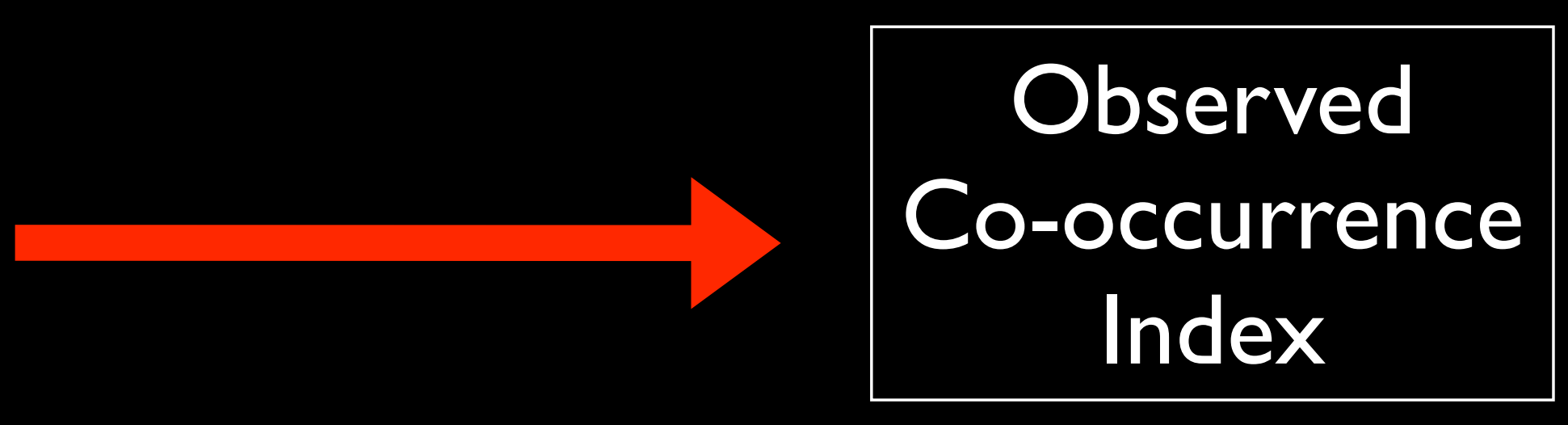




\section{Co-occurrence Analysis}

Occurrence Matrix

\begin{tabular}{|c|c|c|c|}
\hline Species & ObsI & Obs2 & Obs3 \\
\hline SpI & $\mathrm{I}$ & $\mathrm{I}$ & 0 \\
\hline Sp2 & 0 & 0 & $\mathrm{I}$ \\
\hline Sp3 & 0 & $\mathrm{I}$ & $\mathrm{I}$ \\
\hline
\end{tabular}
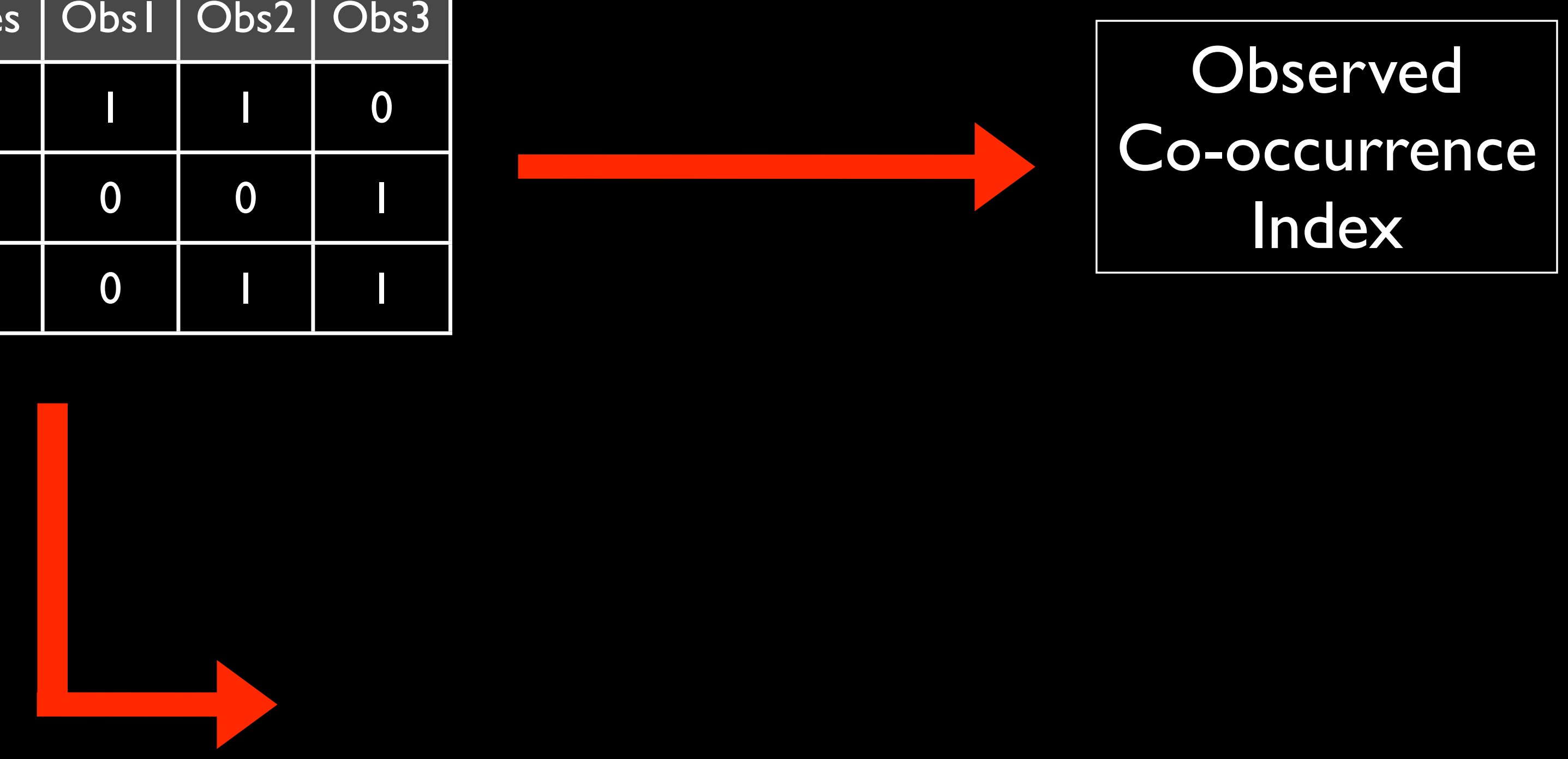

Permutations 


\section{Co-occurrence Analysis}

Occurrence Matrix

\begin{tabular}{|c|c|c|c|}
\hline Species & ObsI & Obs2 & Obs3 \\
\hline SpI & $\mathrm{I}$ & $\mathrm{I}$ & 0 \\
\hline Sp2 & 0 & 0 & $\mathrm{I}$ \\
\hline Sp3 & 0 & $\mathrm{I}$ & $\mathrm{I}$ \\
\hline
\end{tabular}
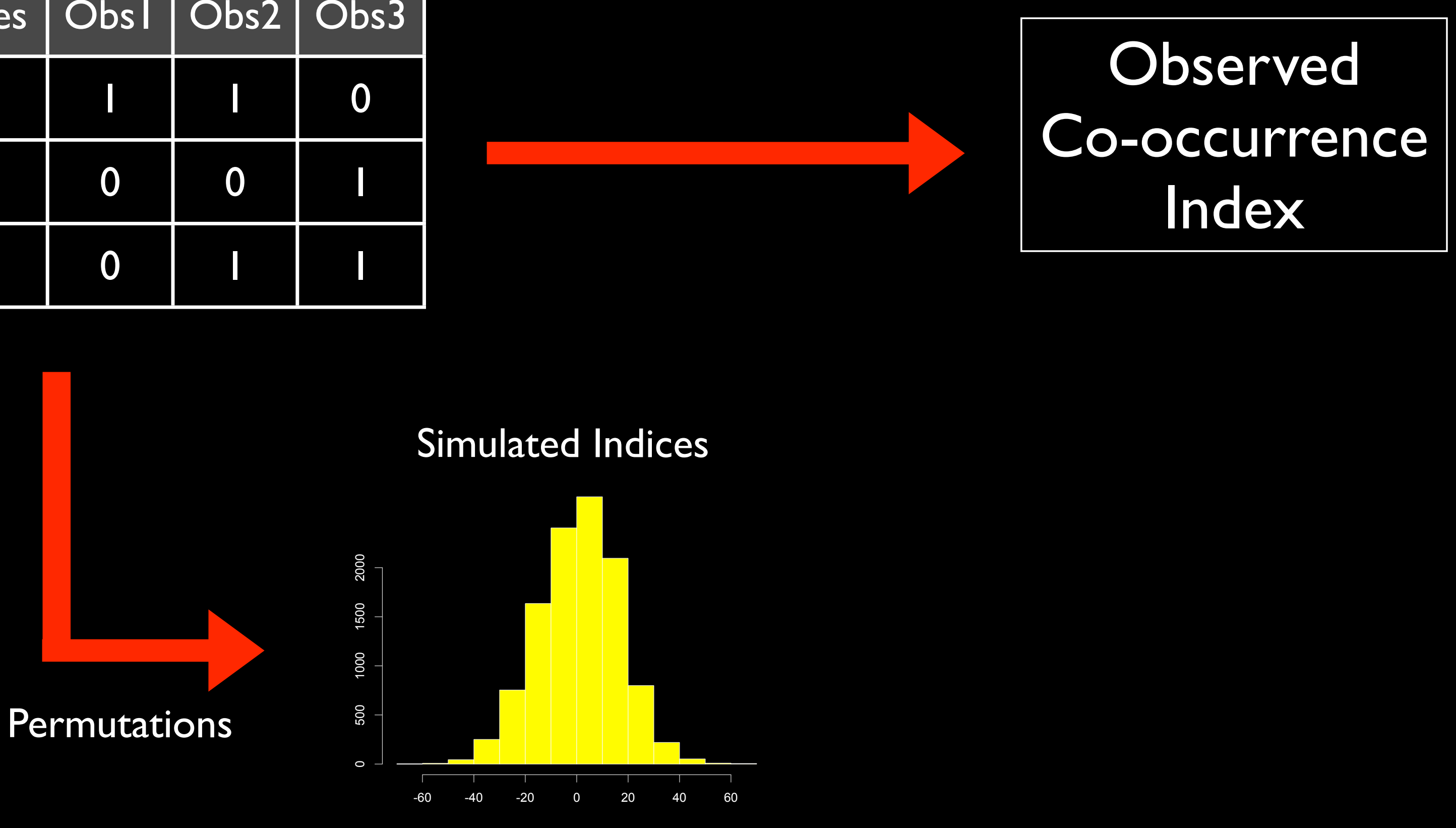


\section{Co-occurrence Analysis}

Occurrence Matrix

\begin{tabular}{|c|c|c|c|}
\hline Species & ObsI & Obs2 & Obs3 \\
\hline SpI & $\mathrm{I}$ & $\mathrm{I}$ & 0 \\
\hline Sp2 & 0 & 0 & $\mathrm{I}$ \\
\hline Sp3 & 0 & $\mathrm{I}$ & $\mathrm{I}$ \\
\hline
\end{tabular}

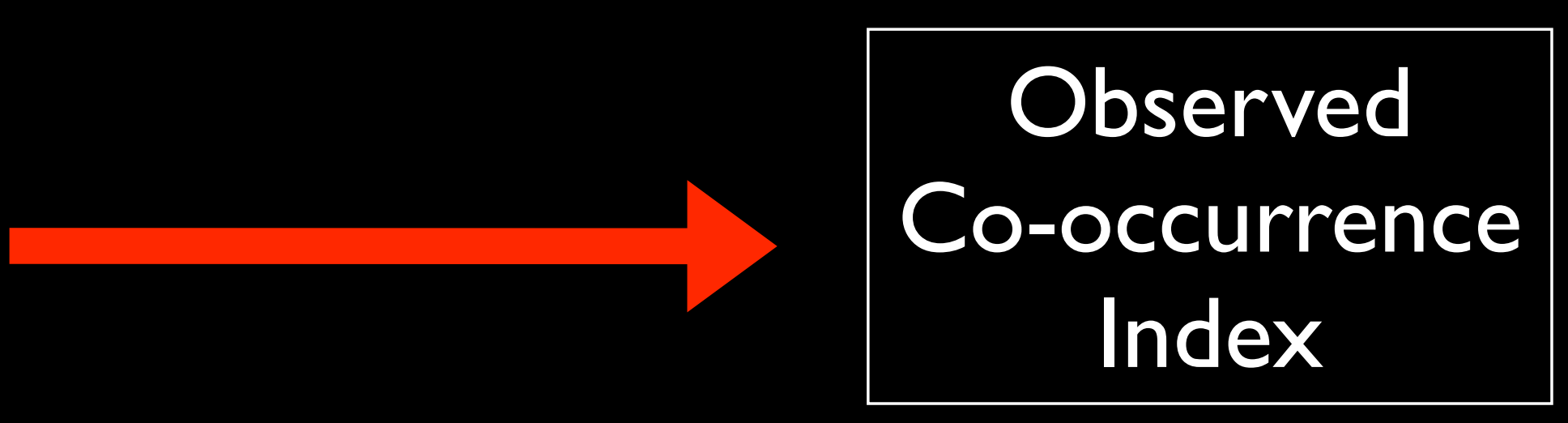

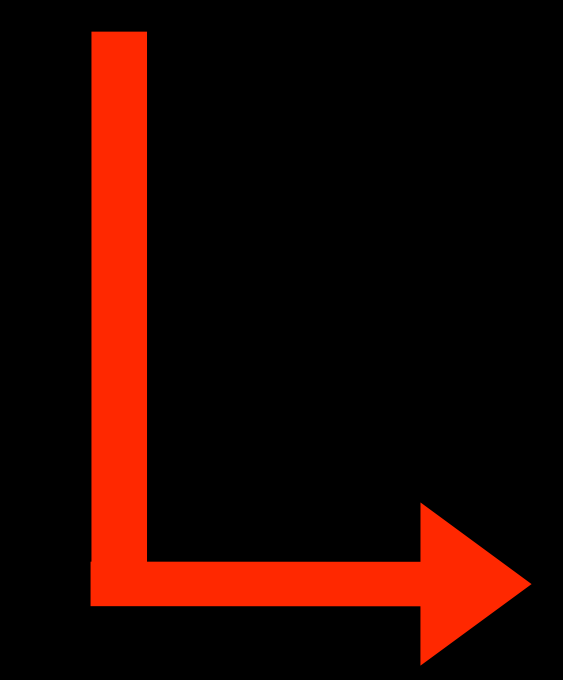

Permutations
Simulated Indices

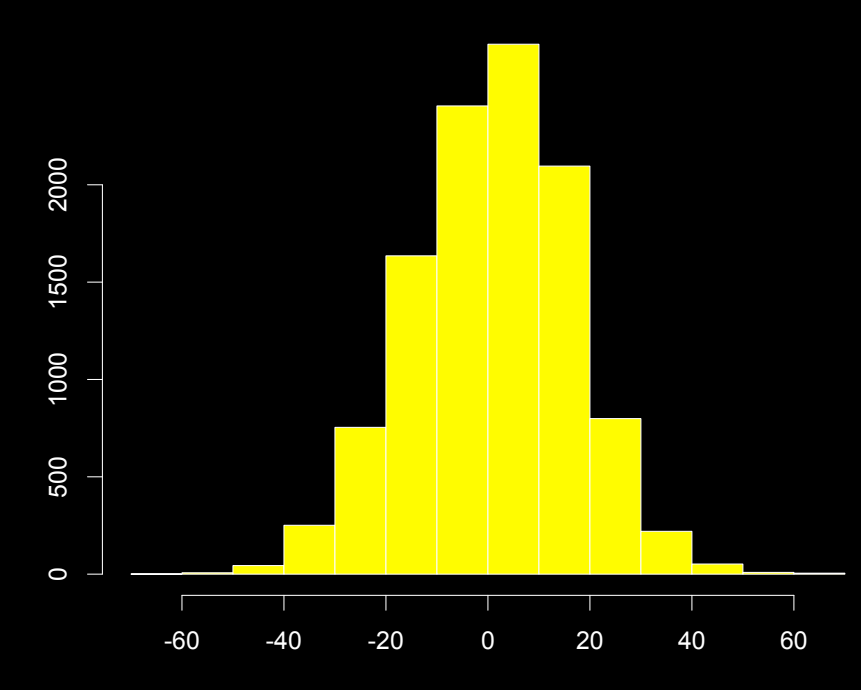

Null Model Index Estimate

Gotelli and Graves 1996 


\section{Co-occurrence Analysis}

Occurrence Matrix

\begin{tabular}{|c|c|c|c|}
\hline Species & ObsI & Obs2 & Obs3 \\
\hline SpI & $\mathrm{I}$ & $\mathrm{I}$ & 0 \\
\hline Sp2 & 0 & 0 & $\mathrm{I}$ \\
\hline Sp3 & 0 & $\mathrm{I}$ & $\mathrm{I}$ \\
\hline
\end{tabular}
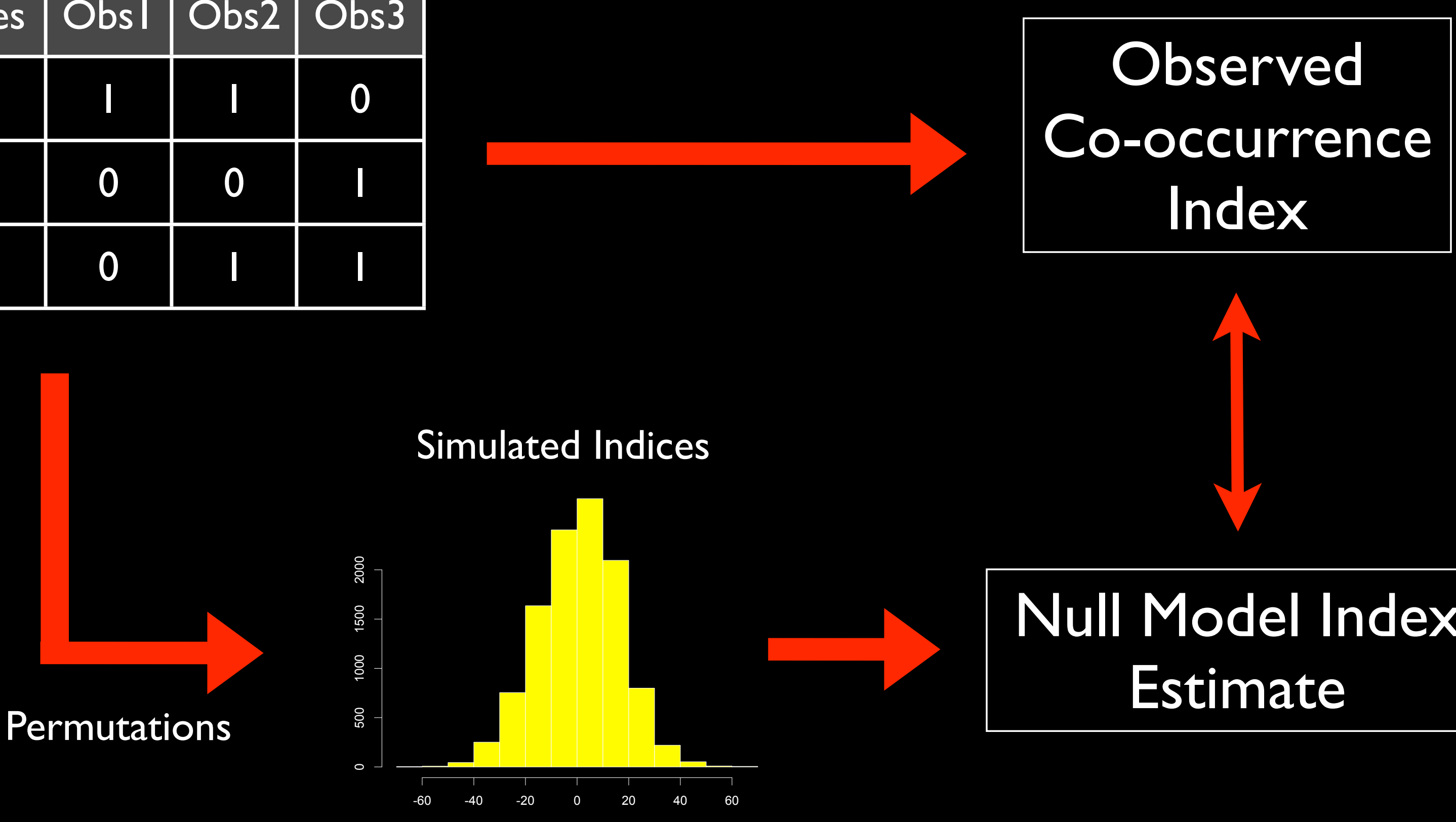

Null Model Index Estimate 


\section{Co-occurrence Analysis}

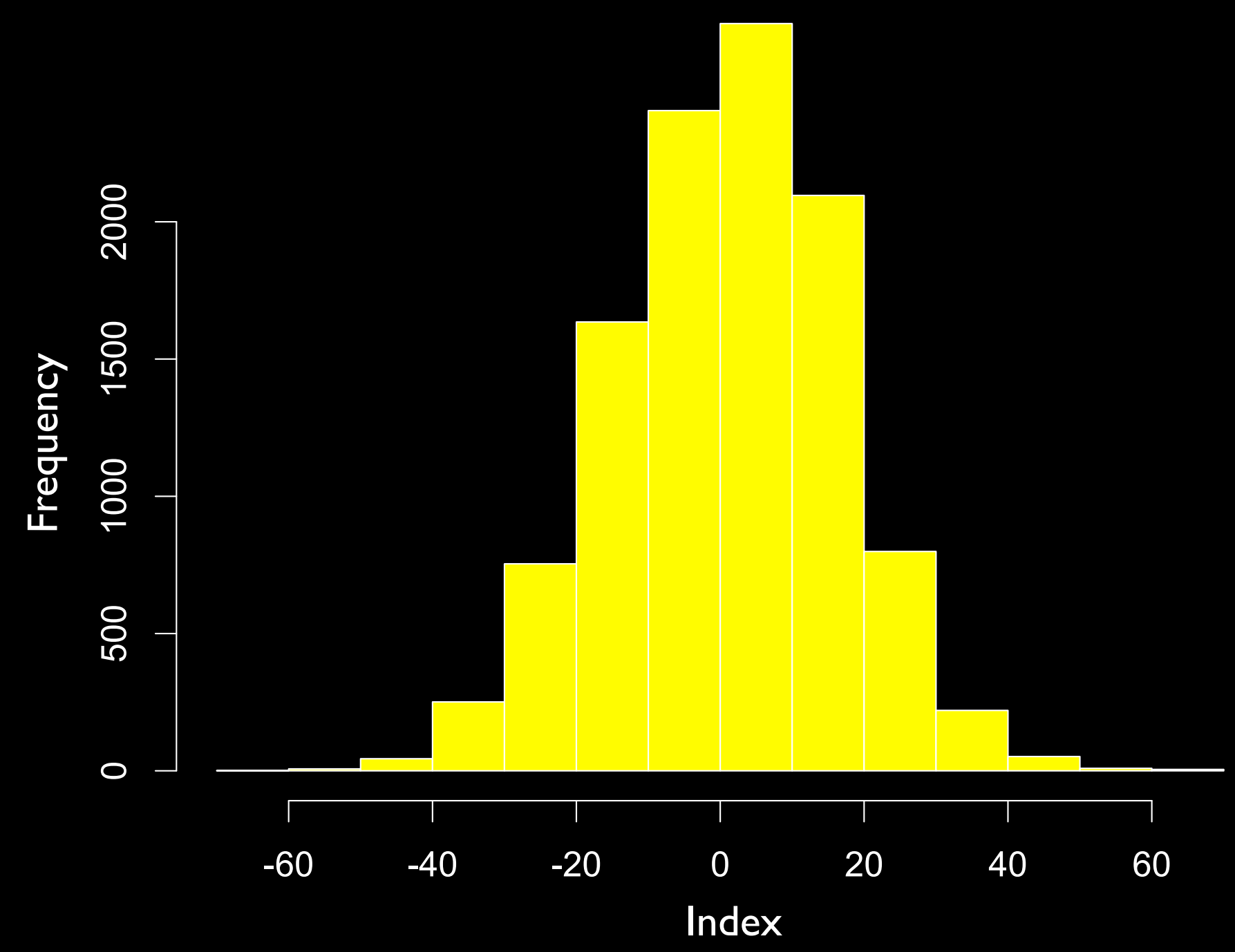




\section{Co-occurrence Analysis}

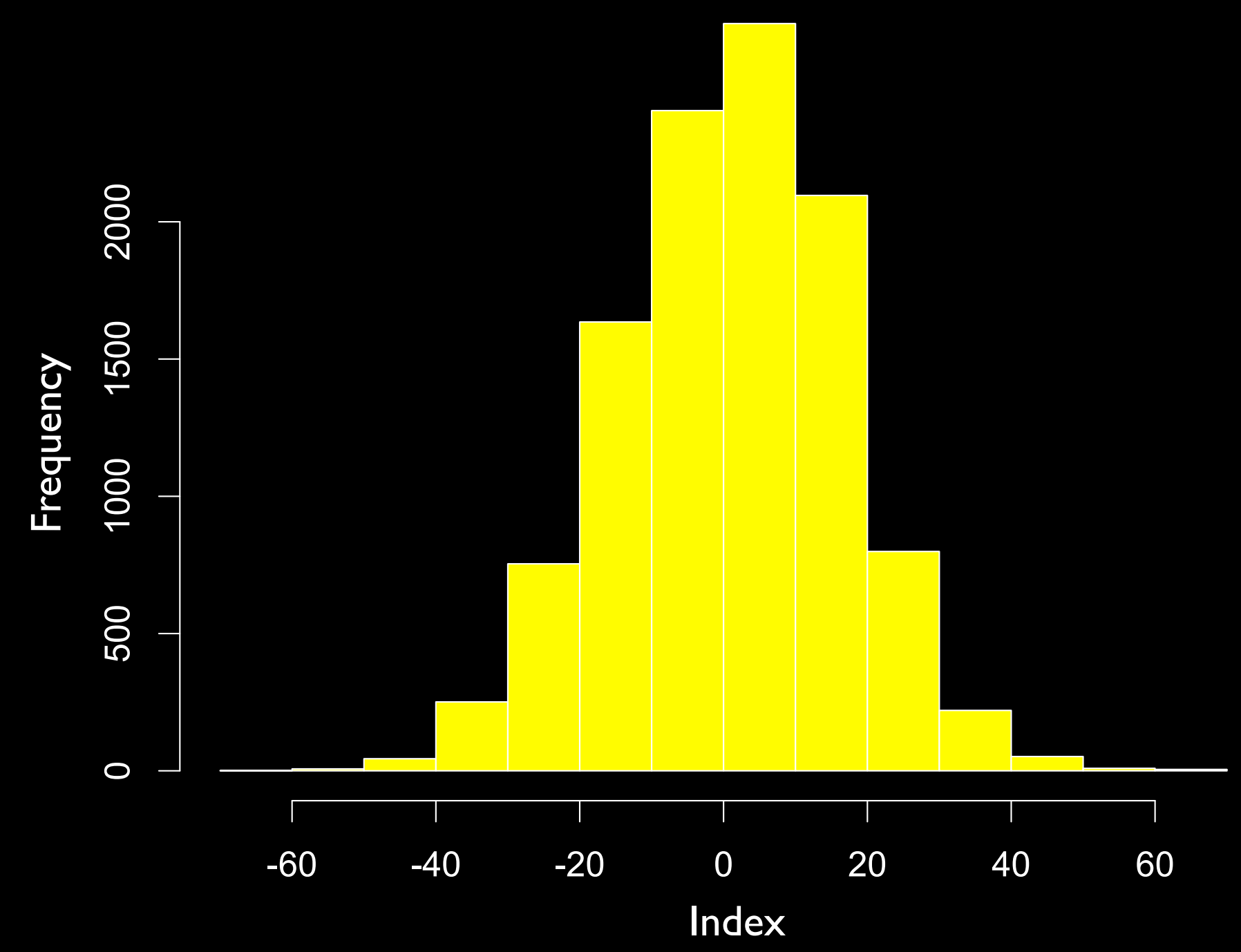

observed index 


\section{Co-occurrence Analysis}

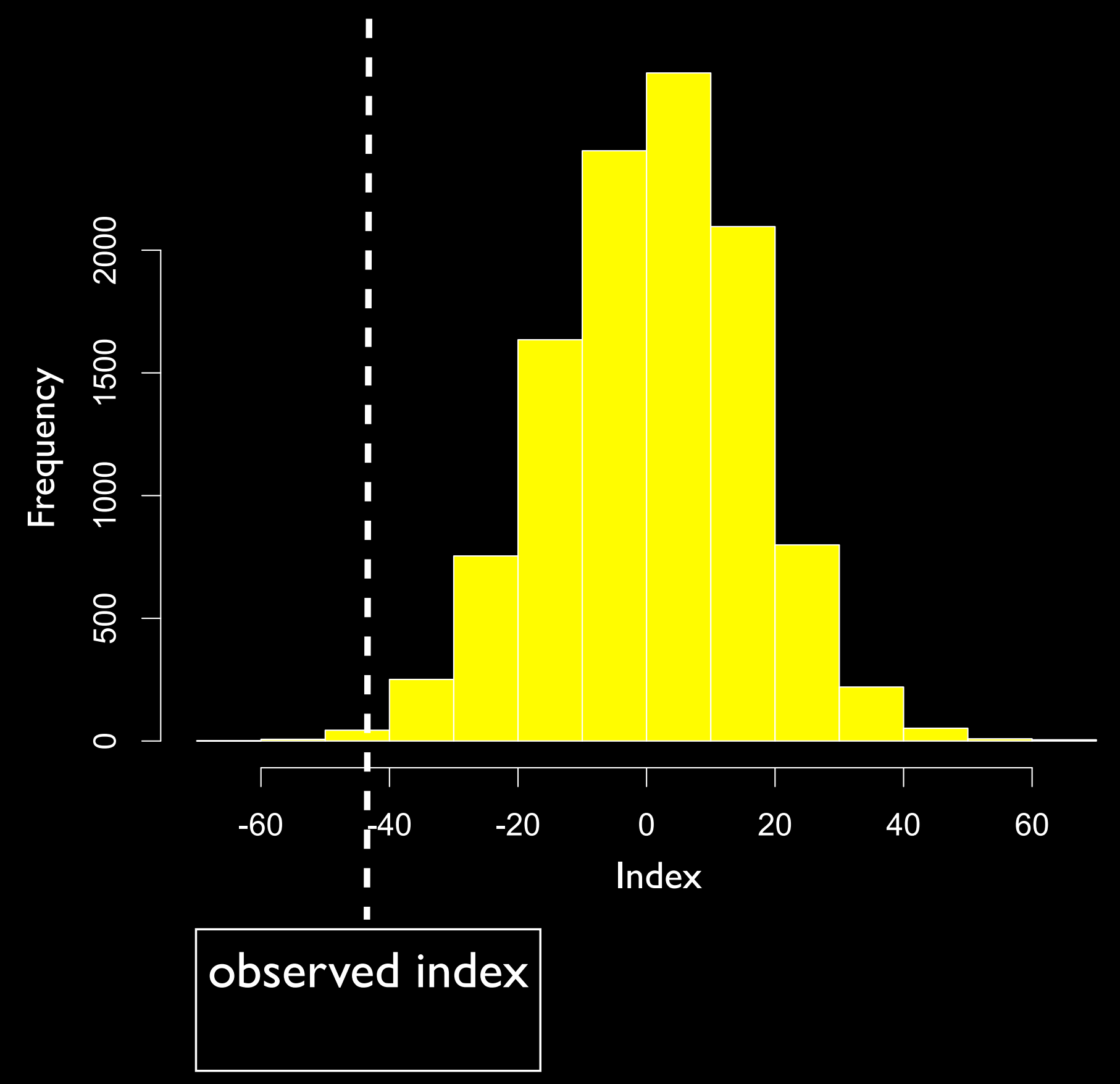




\section{Co-occurrence Analysis}

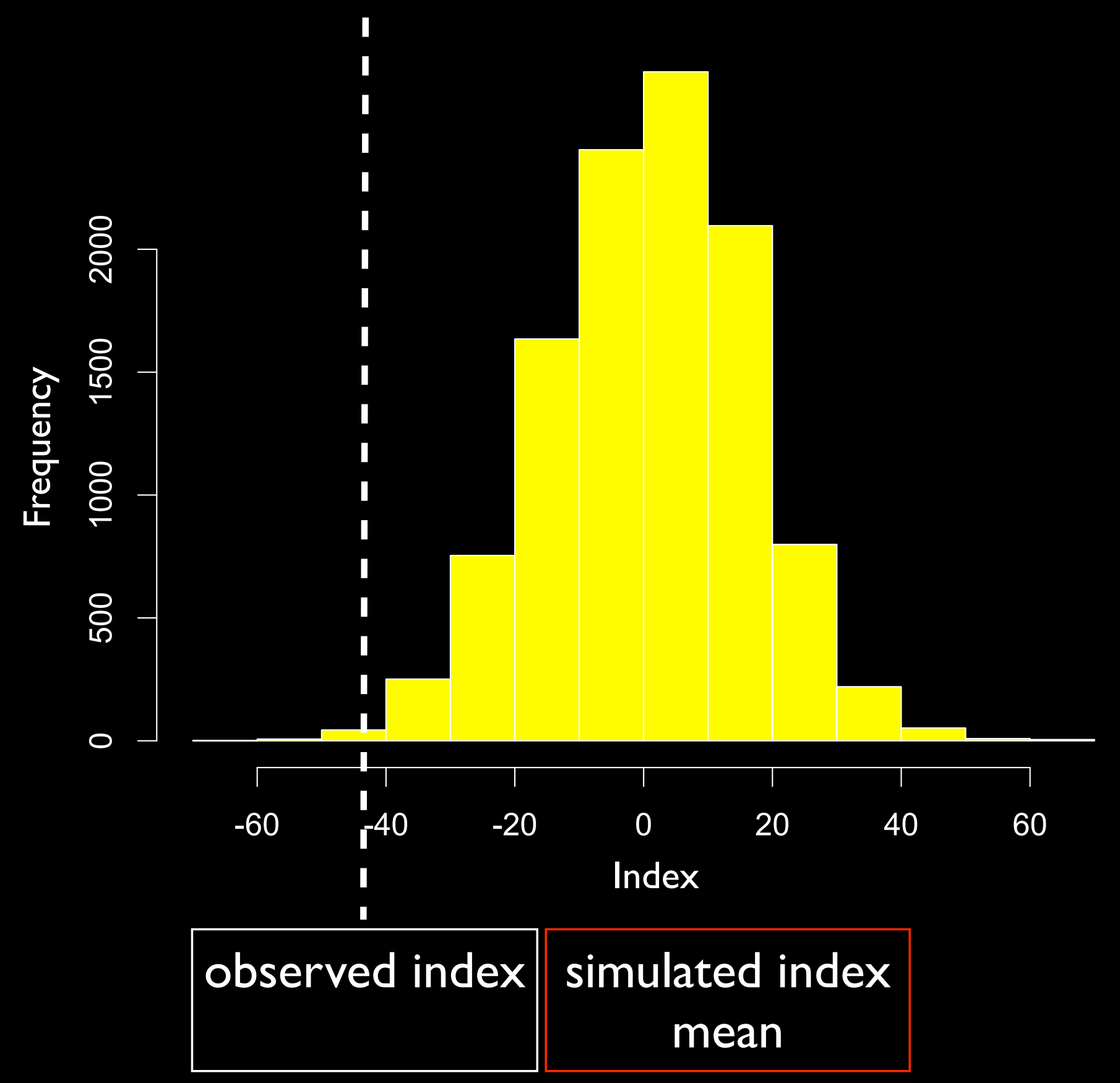




\section{Co-occurrence Analysis}

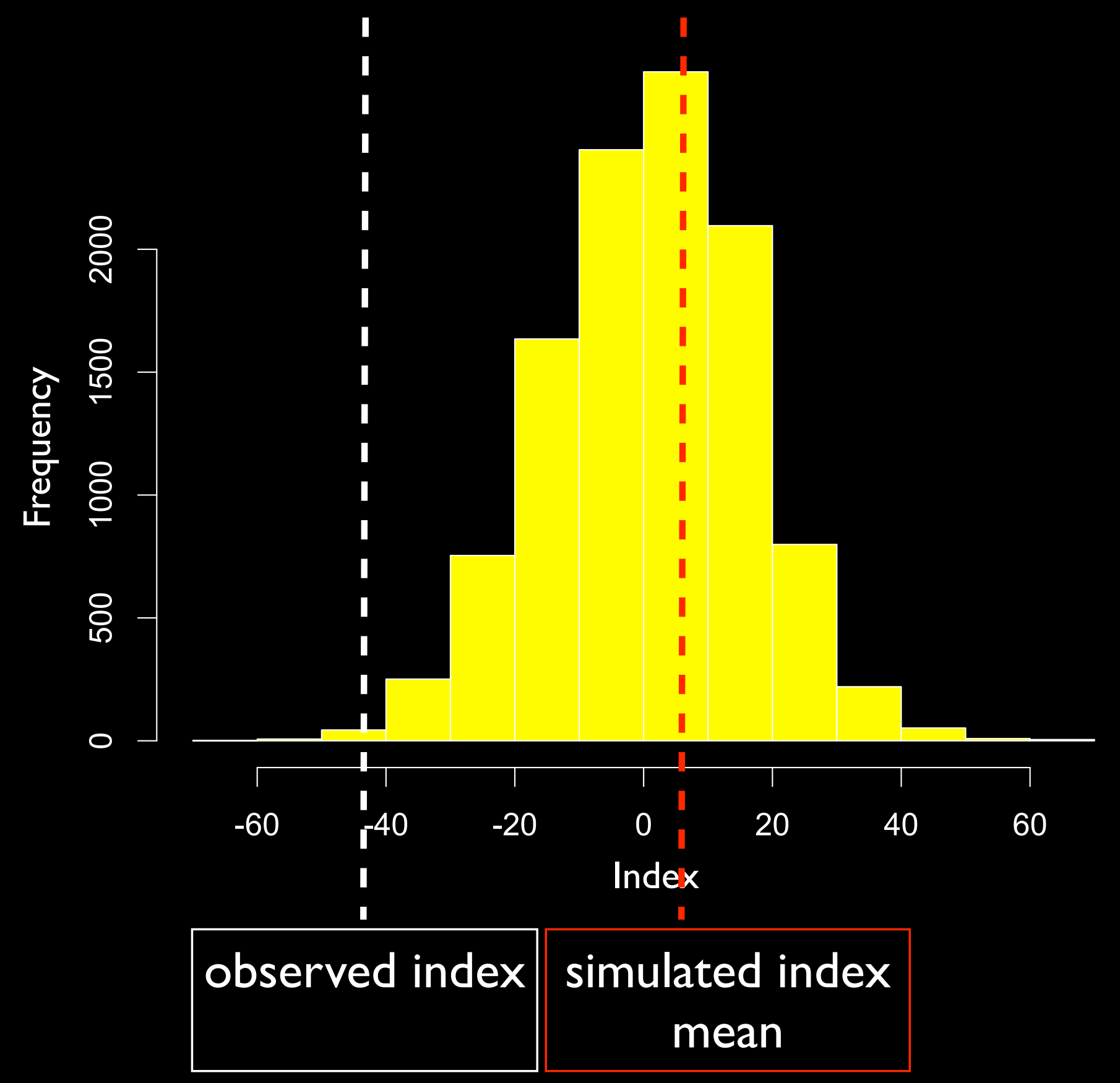




\section{Co-occurrence Analysis}

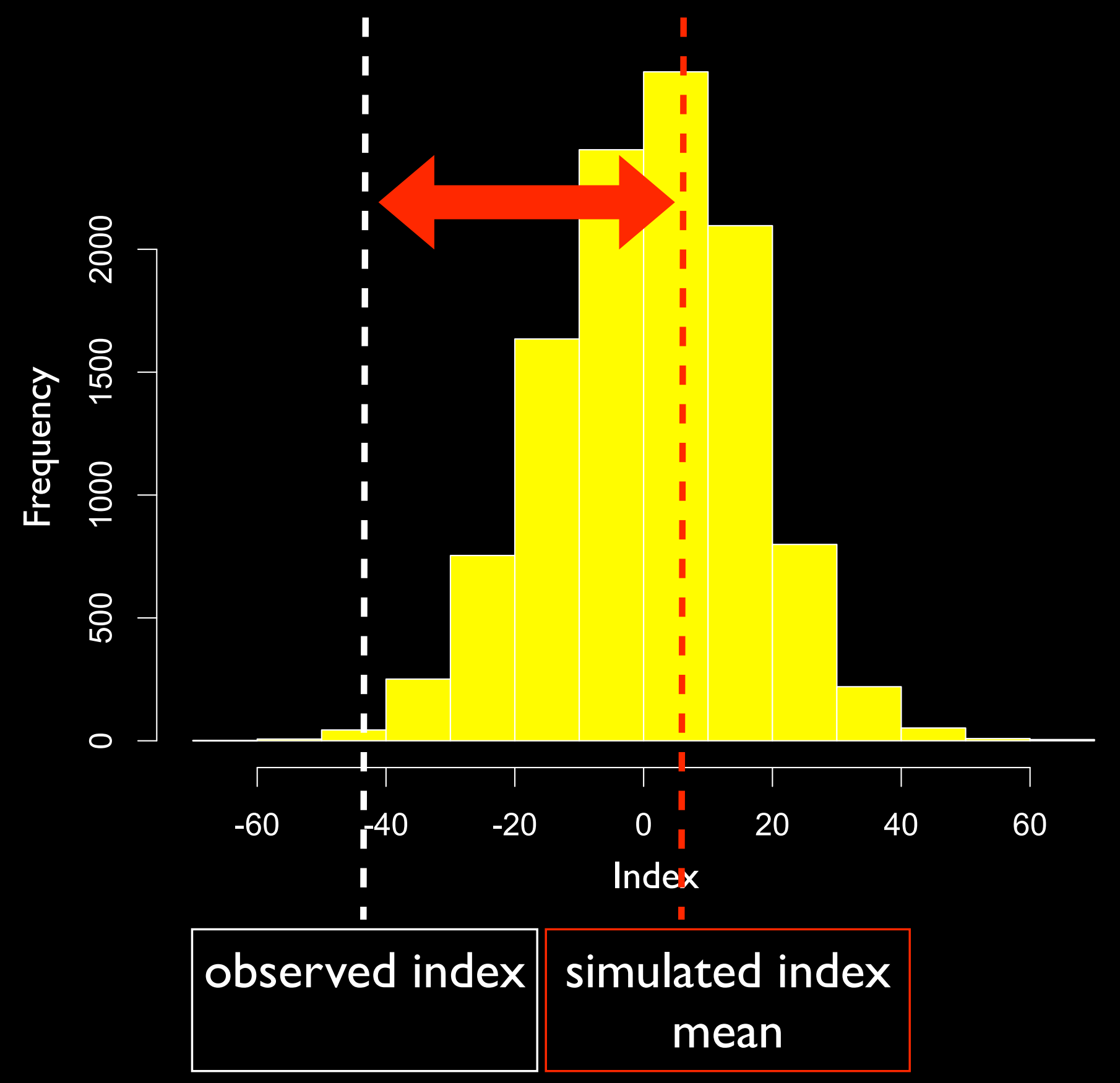




\section{Co-occurrence Analysis}

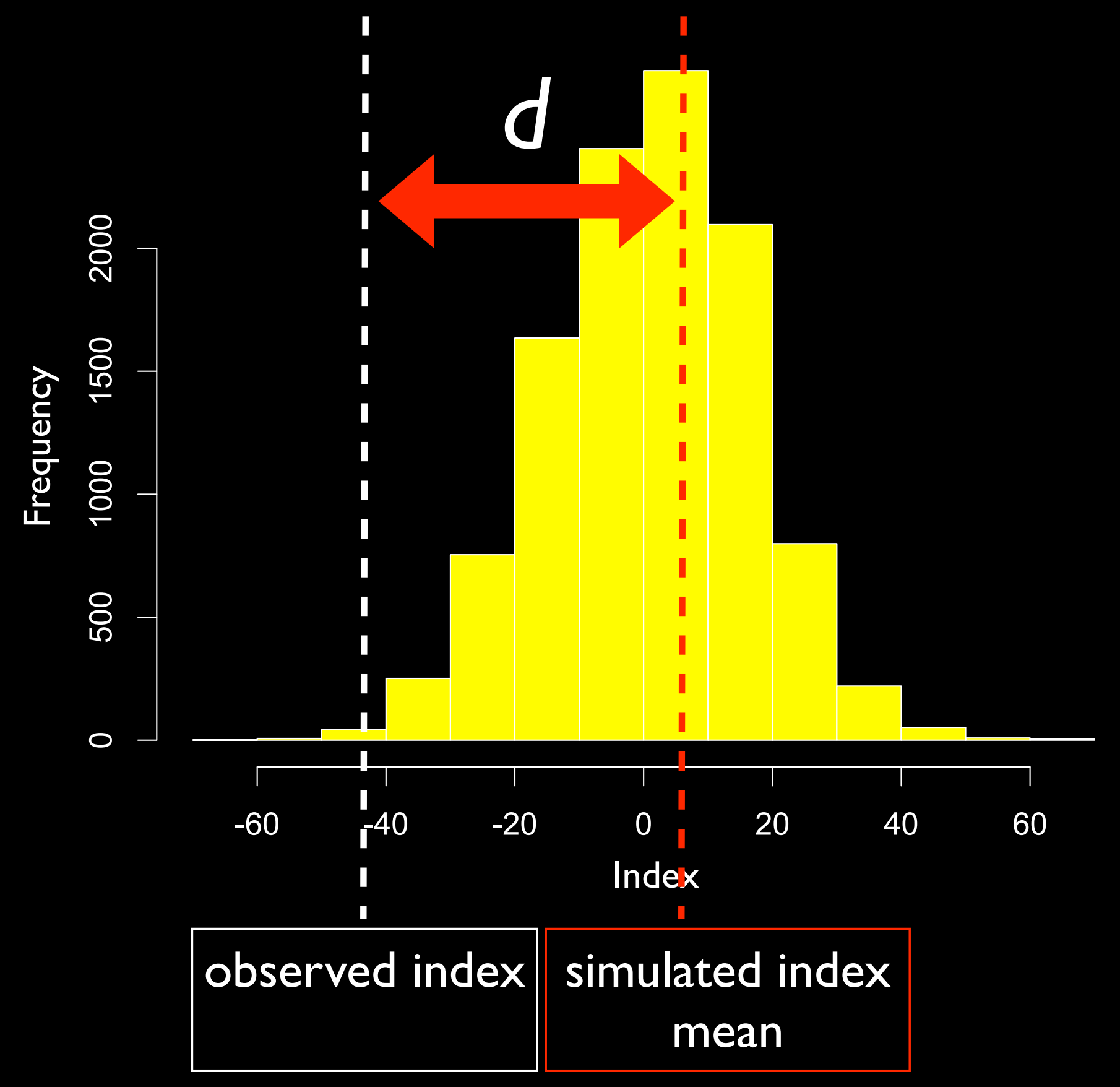




\section{Co-occurrence Analysis}

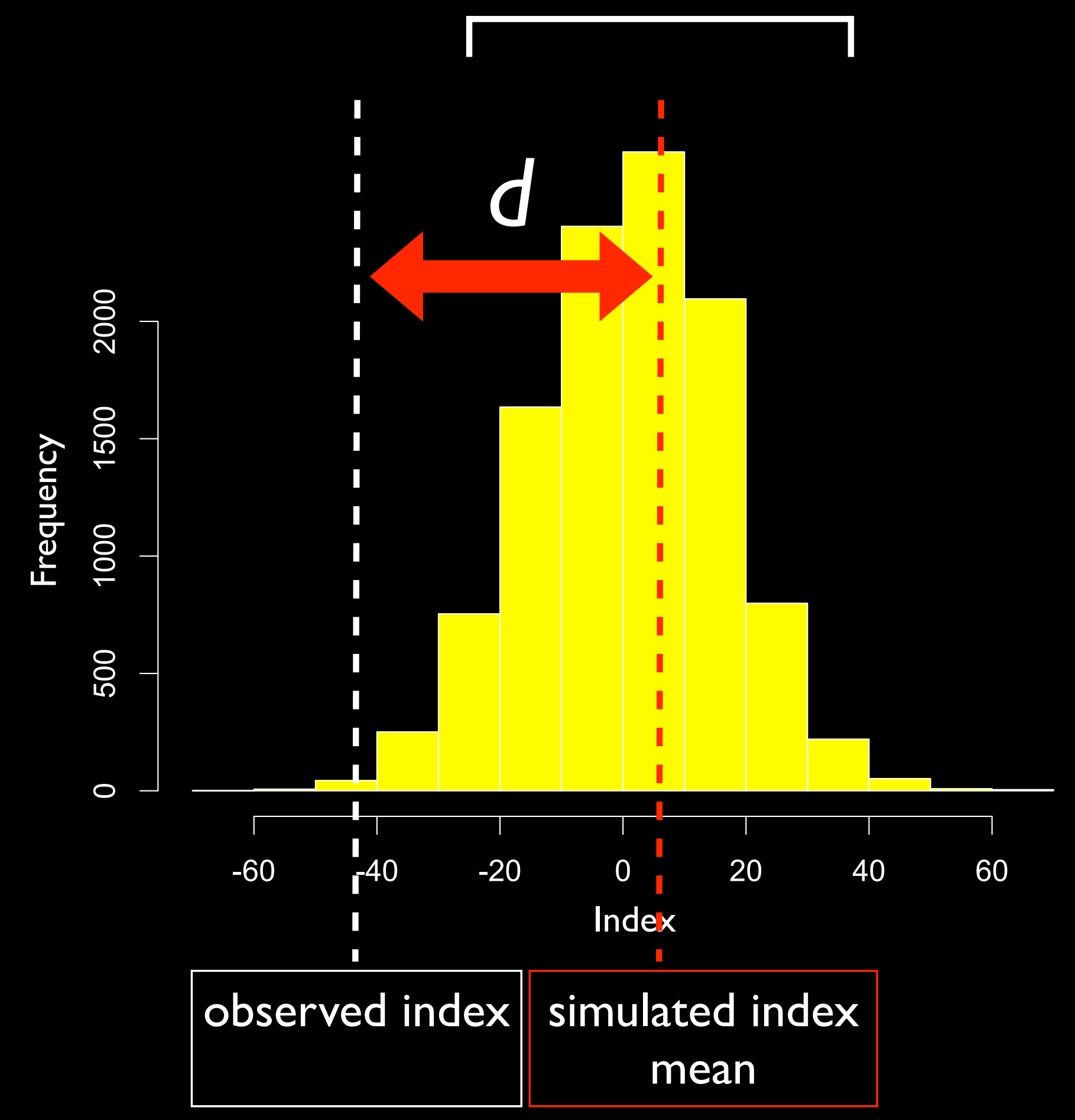




\section{Co-occurrence Analysis}

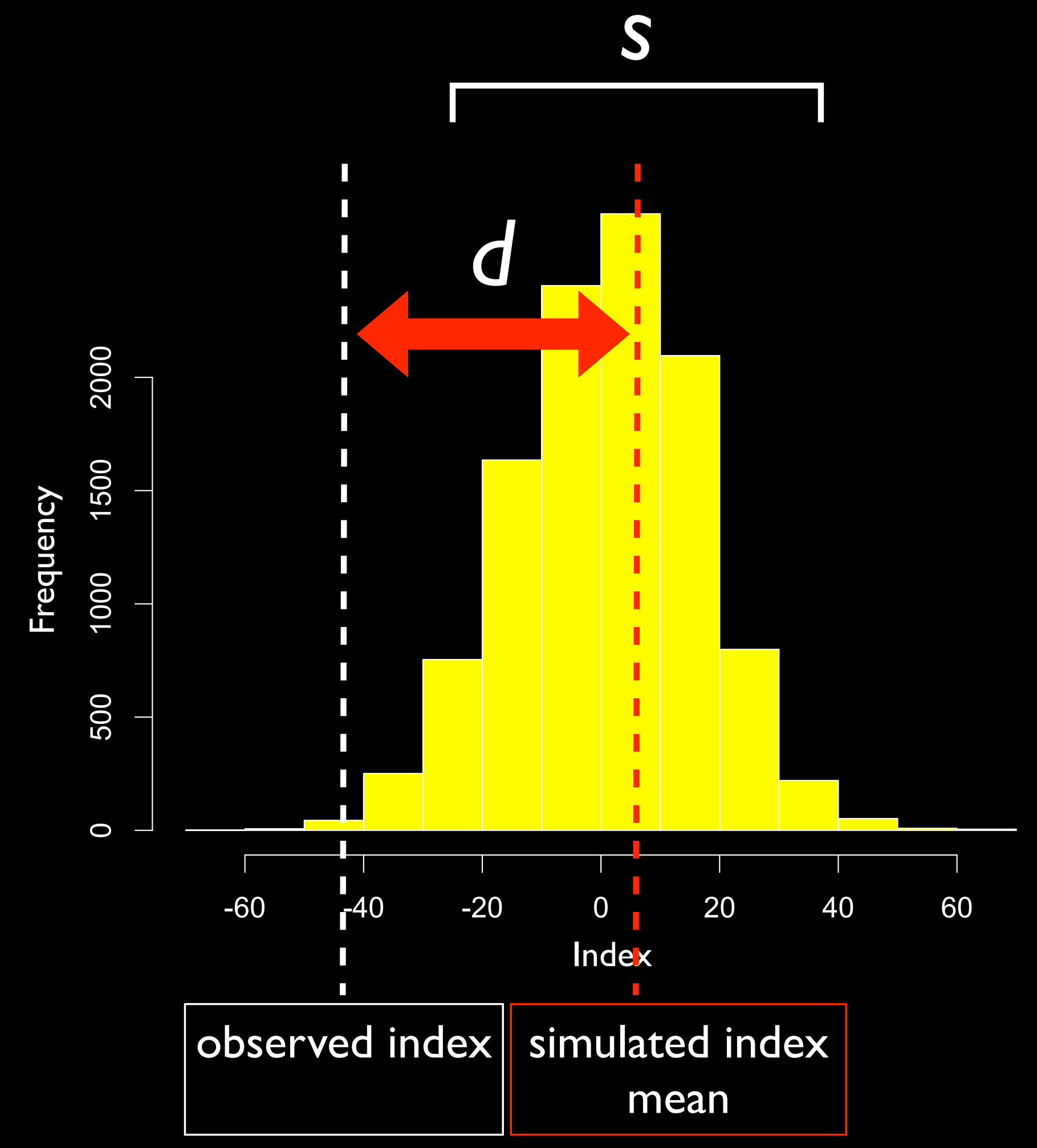




\section{Co-occurrence Analysis}

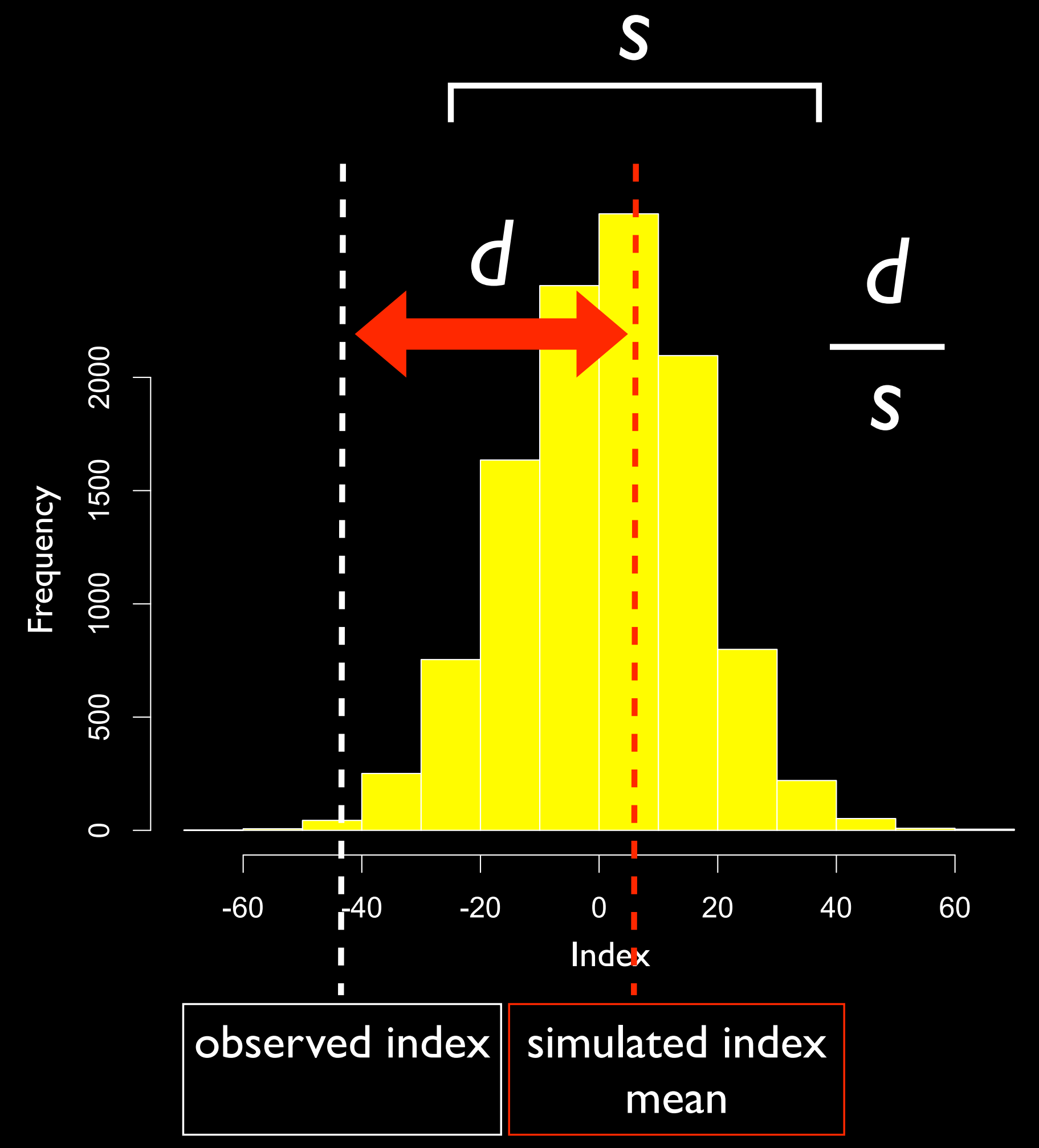




\section{Co-occurrence Analysis}

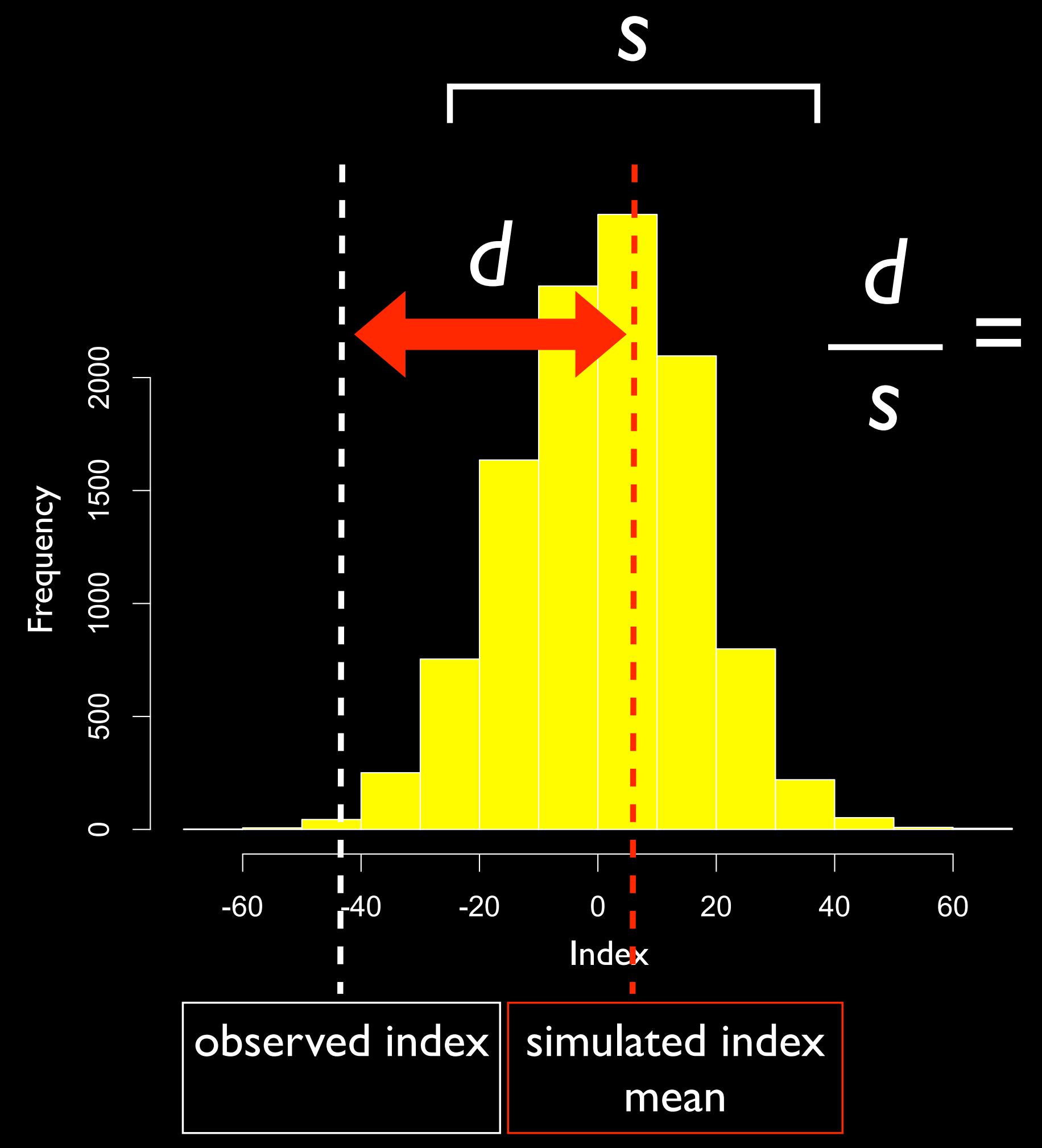




\section{Co-occurrence Analysis}

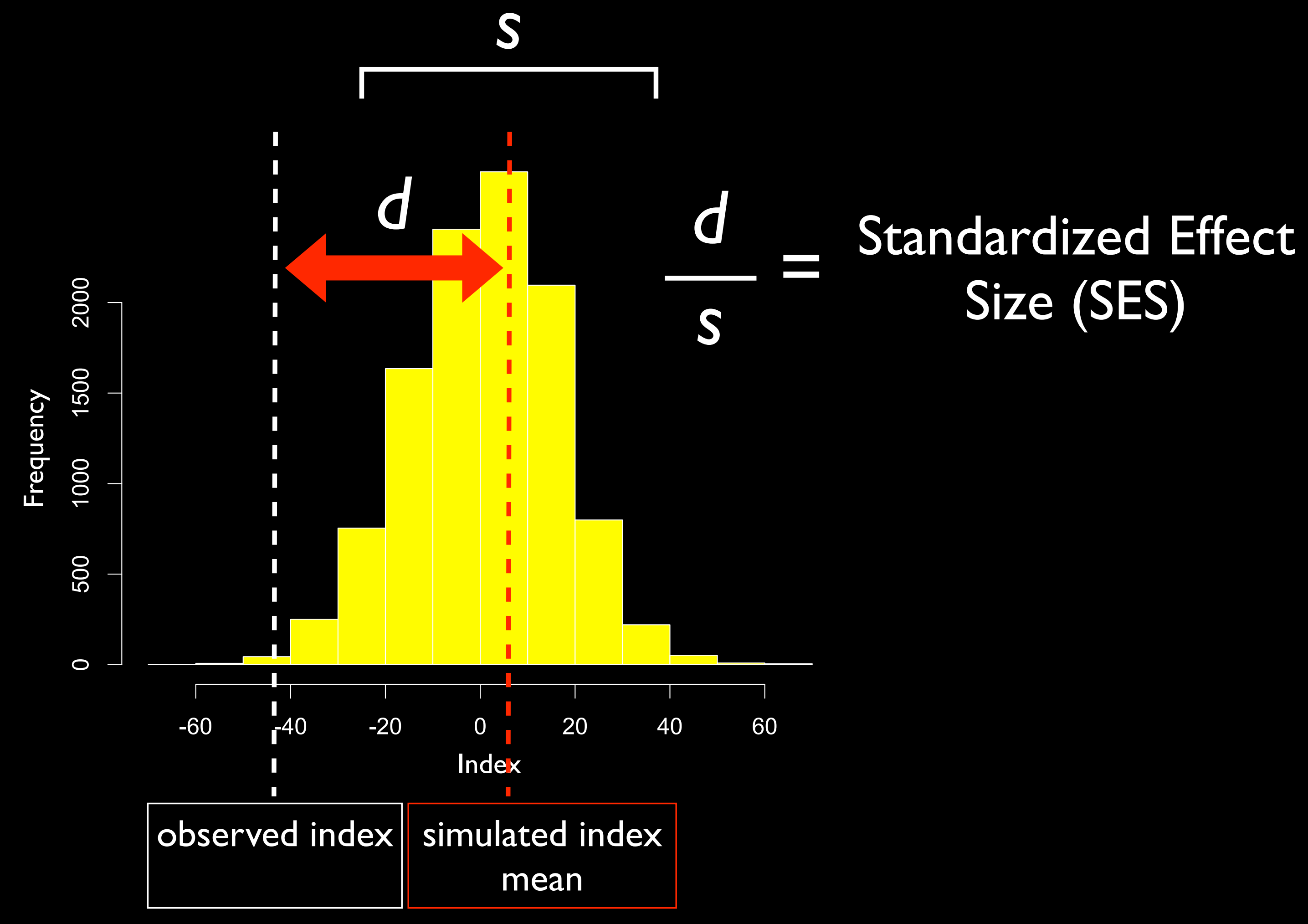


Co-occurrence Analysis 


\section{Co-occurrence Analysis \\ - C-score (Stone and Roberts 1990)}




\section{Co-occurrence Analysis}

- C-score (Stone and Roberts 1990)

- Measures average degree of NON-co-occurrence 


\section{Co-occurrence Analysis}

- C-score (Stone and Roberts 1990)

- Measures average degree of NON-co-occurrence

- Fixed-equiprobable algorithm (Gotelli 2000) 


\section{Co-occurrence Analysis}

- C-score (Stone and Roberts 1990)

- Measures average degree of NON-co-occurrence

- Fixed-equiprobable algorithm (Gotelli 2000)

- Standardized effect size (SES) 


\section{Co-occurrence Analysis}

- C-score (Stone and Roberts 1990)

- Measures average degree of NON-co-occurrence

- Fixed-equiprobable algorithm (Gotelli 2000)

- Standardized effect size (SES)

\section{SES}




\section{Co-occurrence Analysis}

- C-score (Stone and Roberts 1990)

- Measures average degree of NON-co-occurrence

- Fixed-equiprobable algorithm (Gotelli 2000)

- Standardized effect size (SES)

SES 0 Random 


\section{Co-occurrence Analysis}

- C-score (Stone and Roberts 1990)

- Measures average degree of NON-co-occurrence

- Fixed-equiprobable algorithm (Gotelli 2000)

- Standardized effect size (SES)

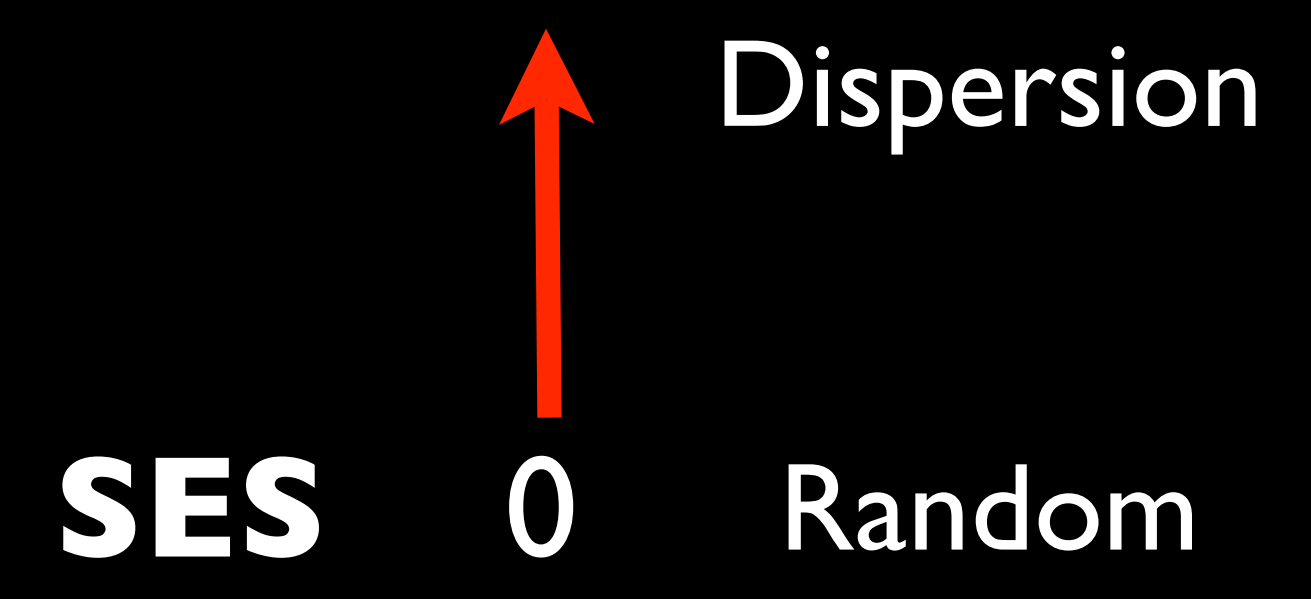




\section{Co-occurrence Analysis}

- C-score (Stone and Roberts 1990)

- Measures average degree of NON-co-occurrence

- Fixed-equiprobable algorithm (Gotelli 2000)

- Standardized effect size (SES)

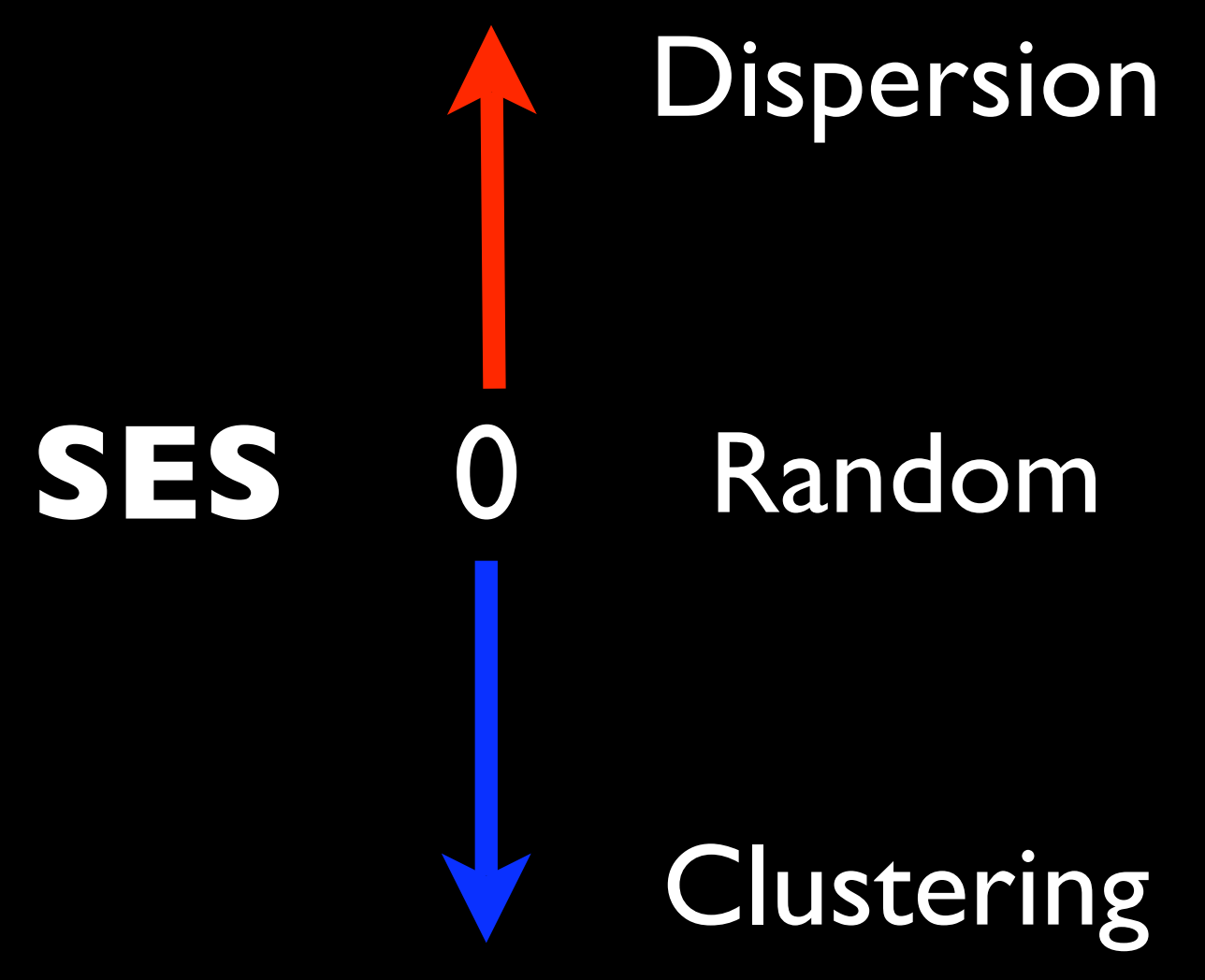




\section{Results}

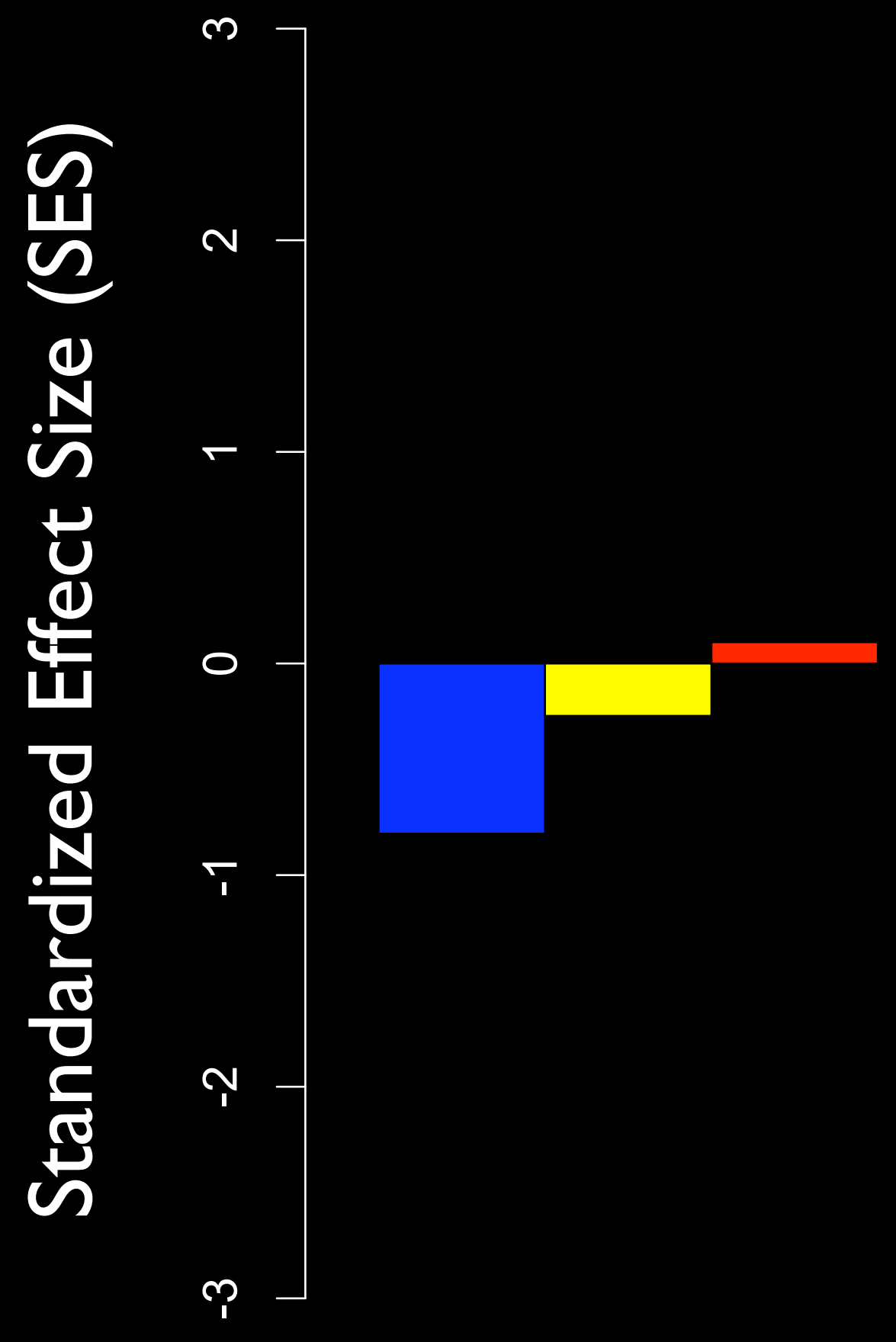

I year
Month

June

August

October

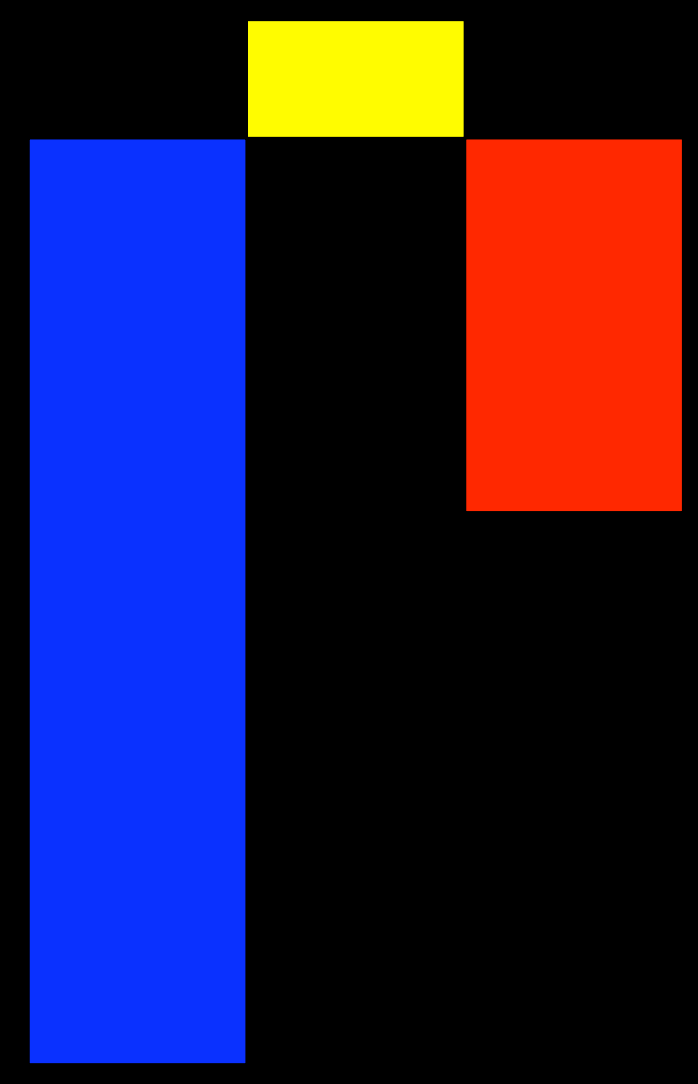

4 years

6 years

STAND AGE 
Results

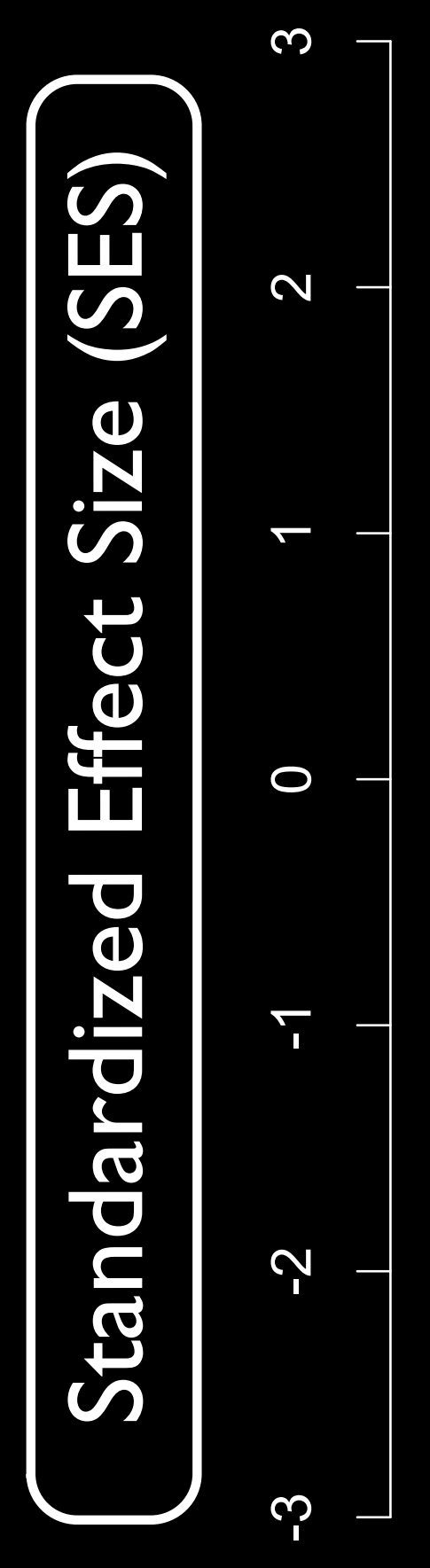

Month

June

a August

October

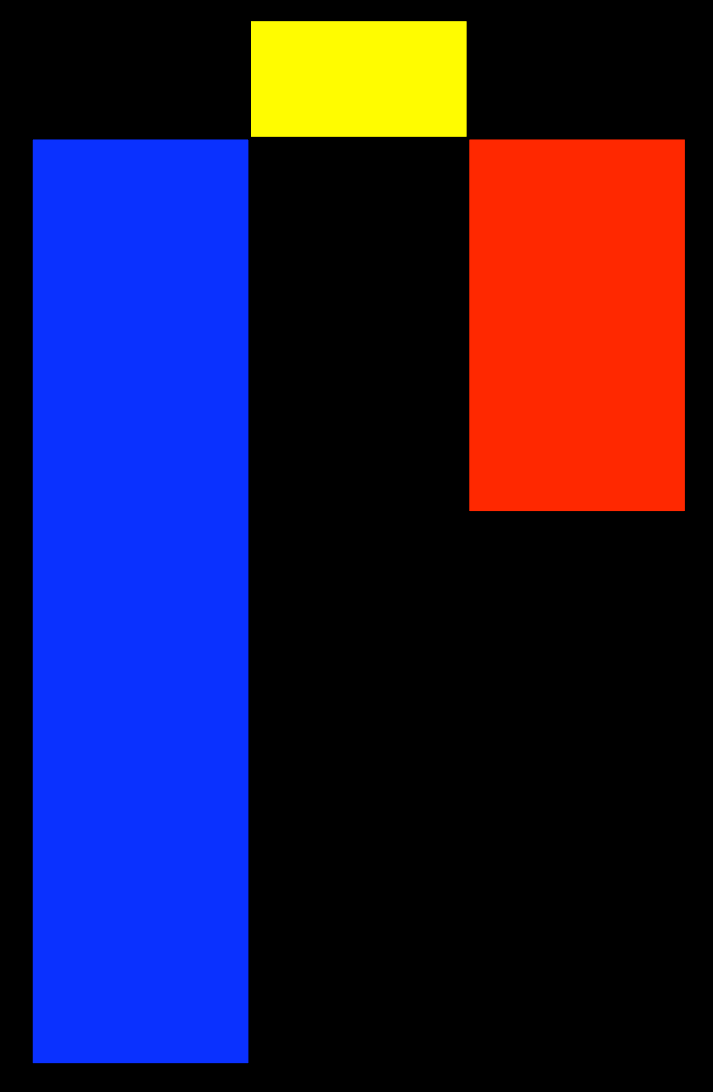

4 years

6 years

year

STAND AGE 


\section{Results}

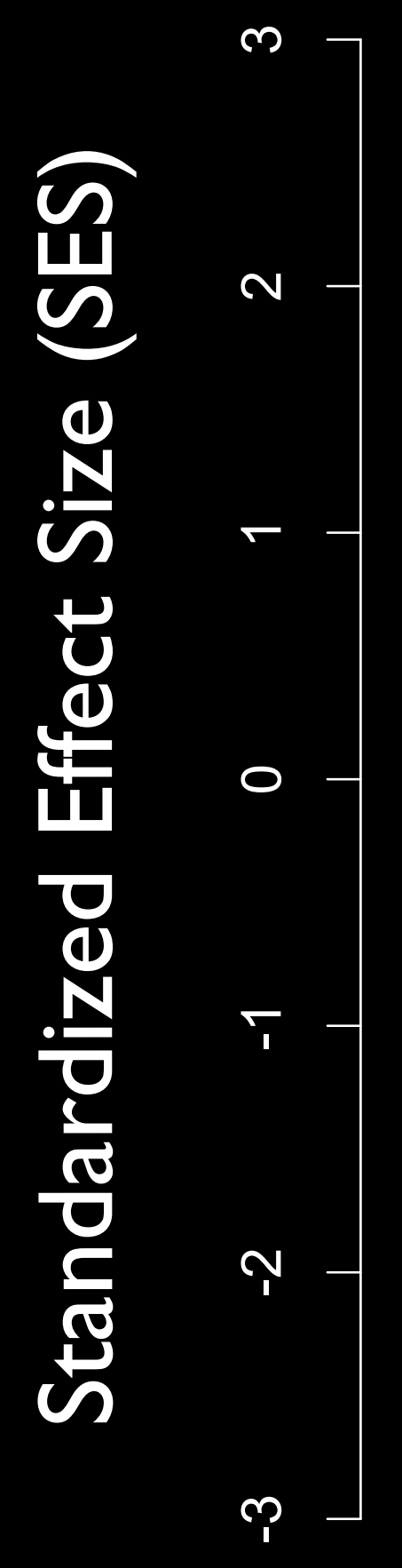

Month

June

august

October

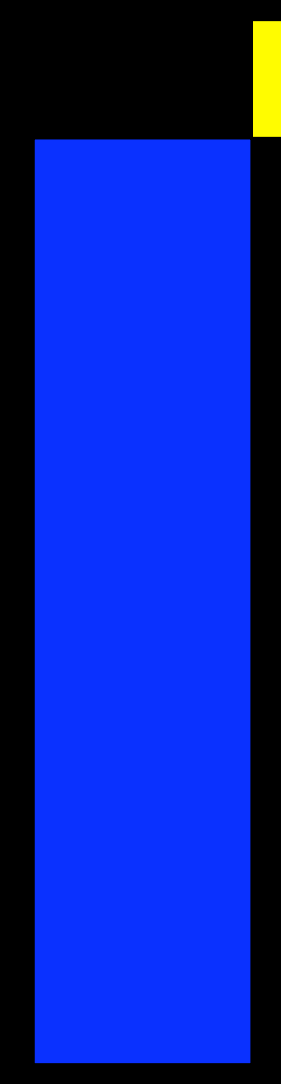

4 years

6 years

STAND AGE 


\section{Results}
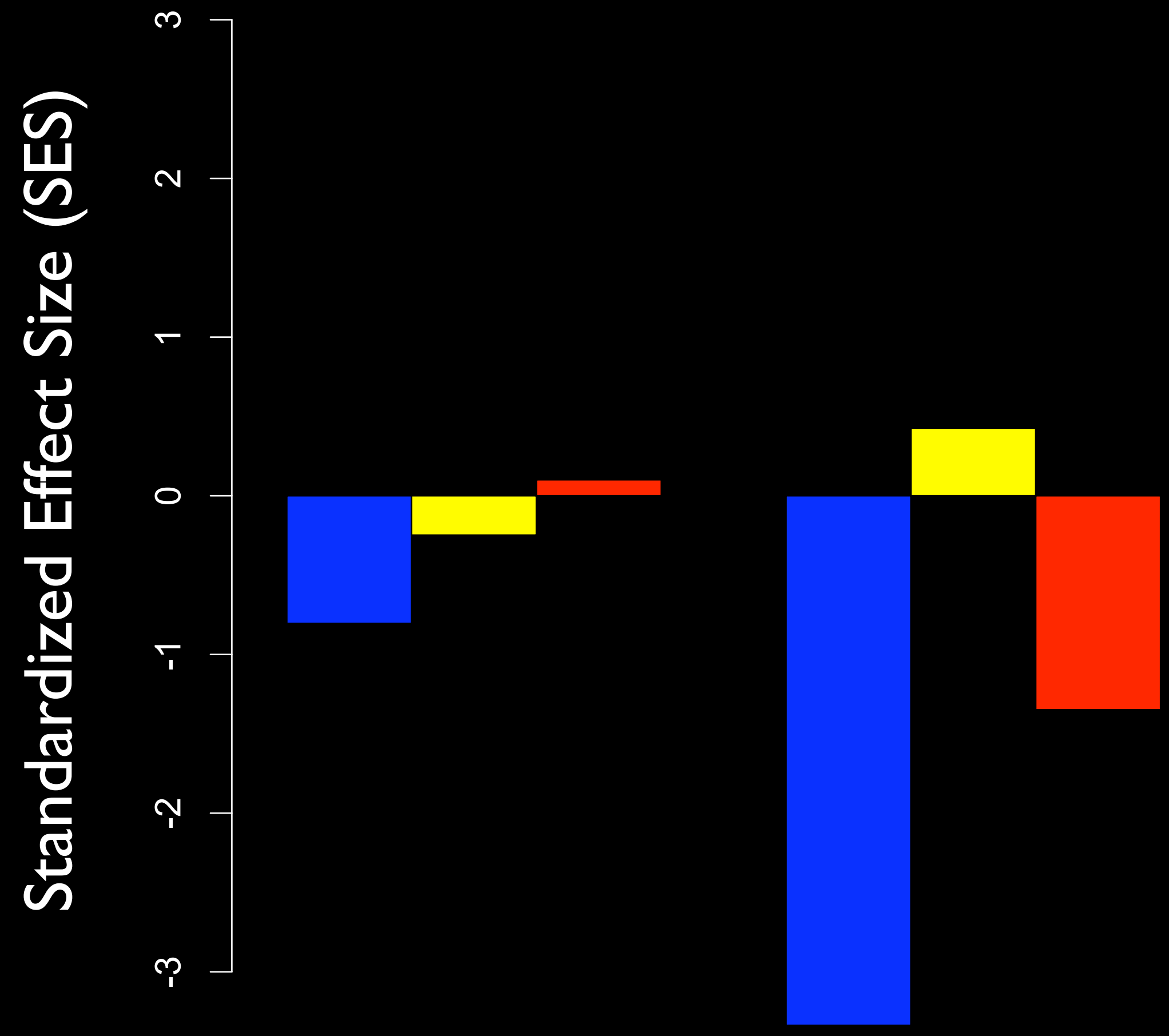

Month

June

a August

$\square$ October

I year

4 years

6 years

STAND AGE 
Results

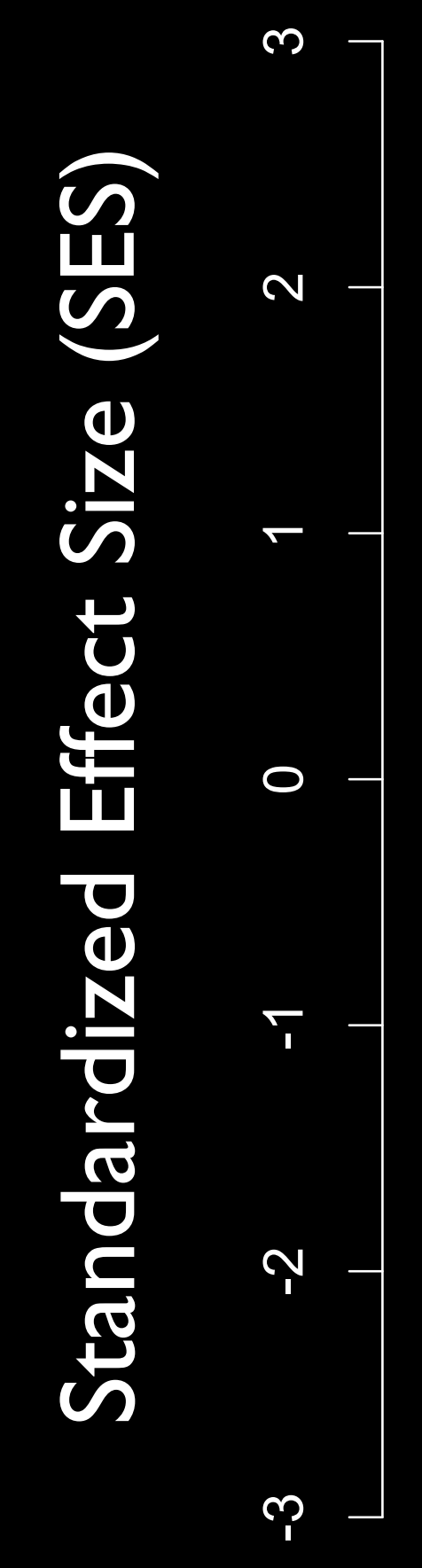

Month
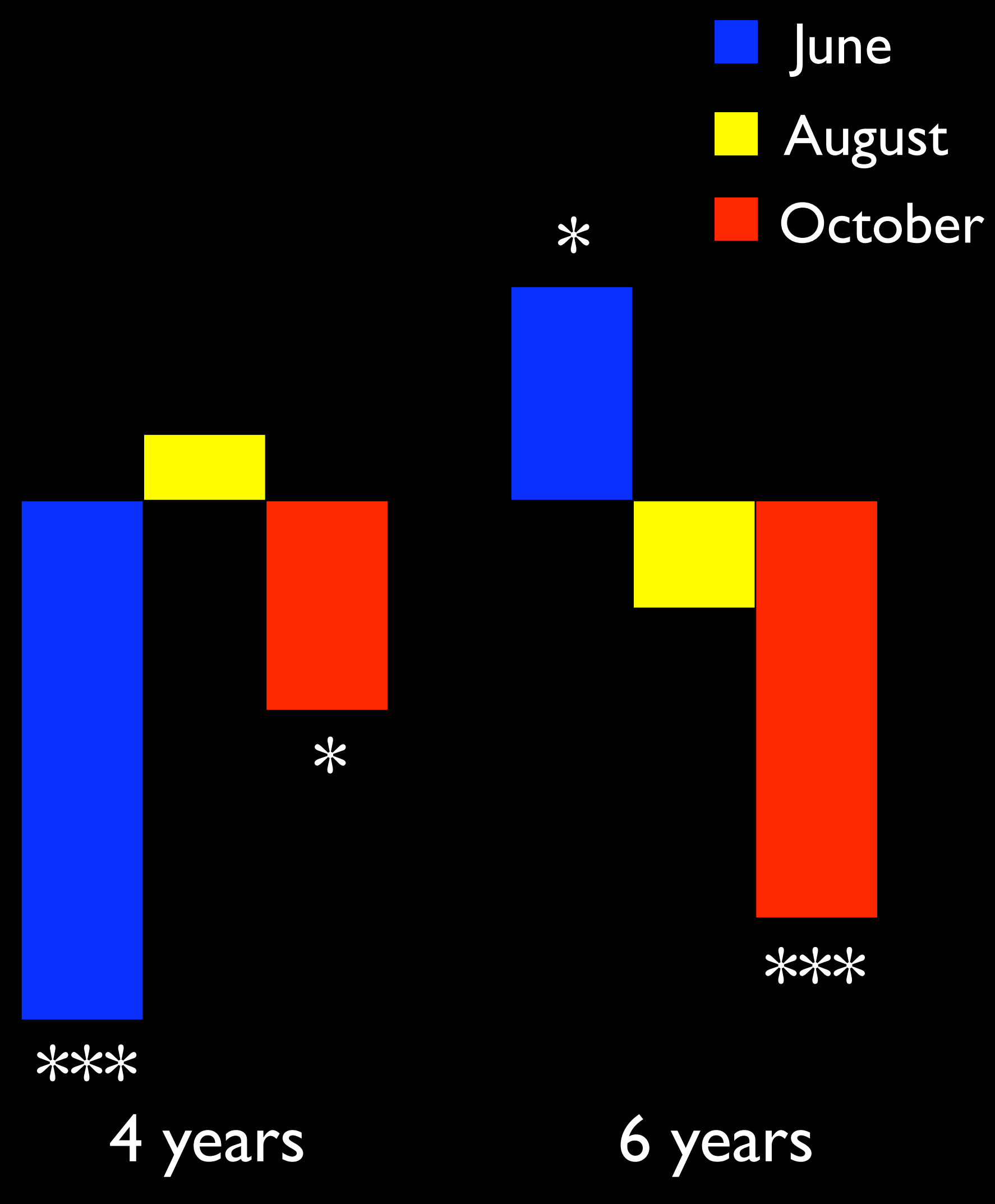

STAND AGE 


\section{Results}

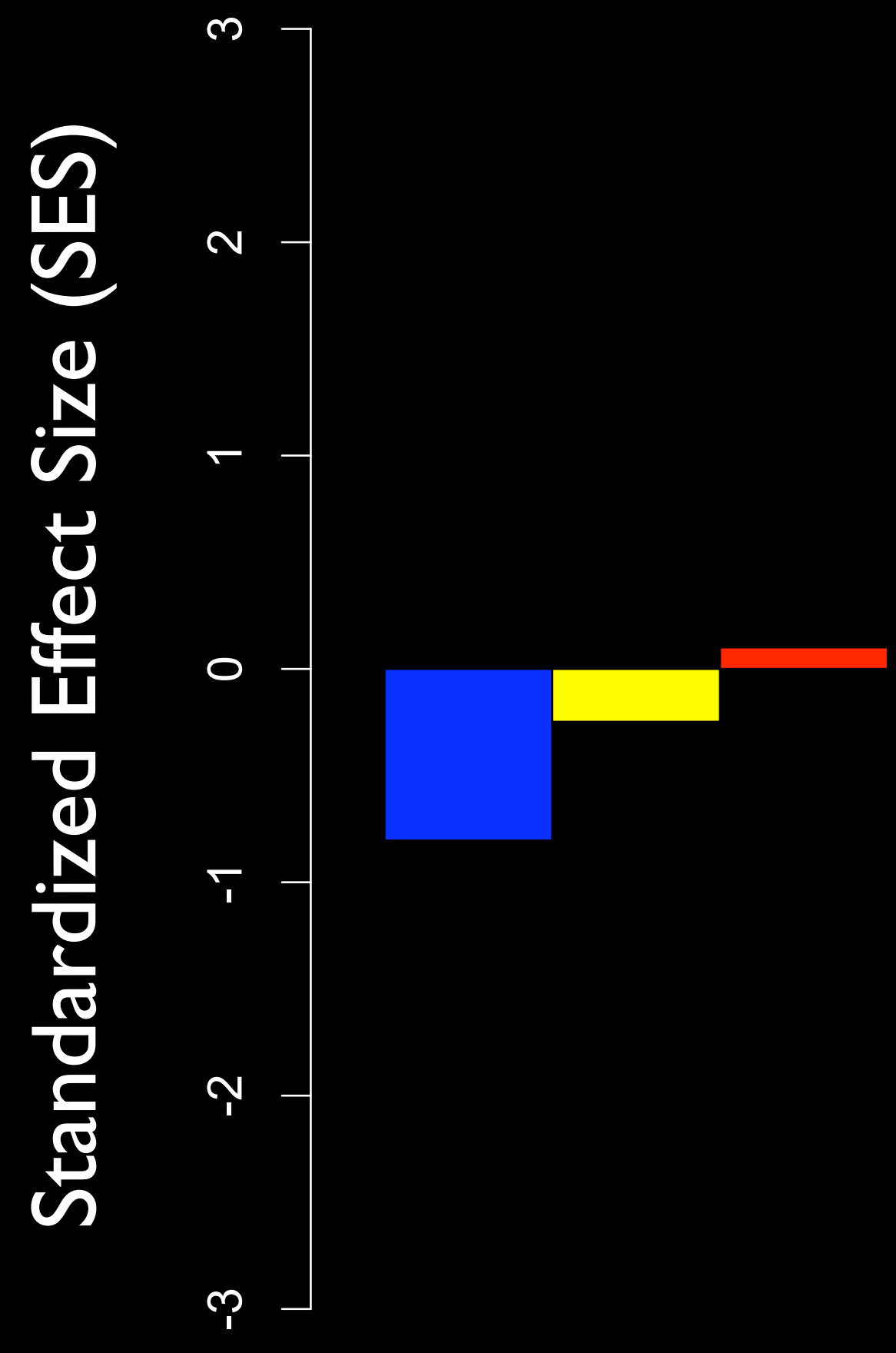

year

$P$-value: $*<0.10, * * *<0.01$

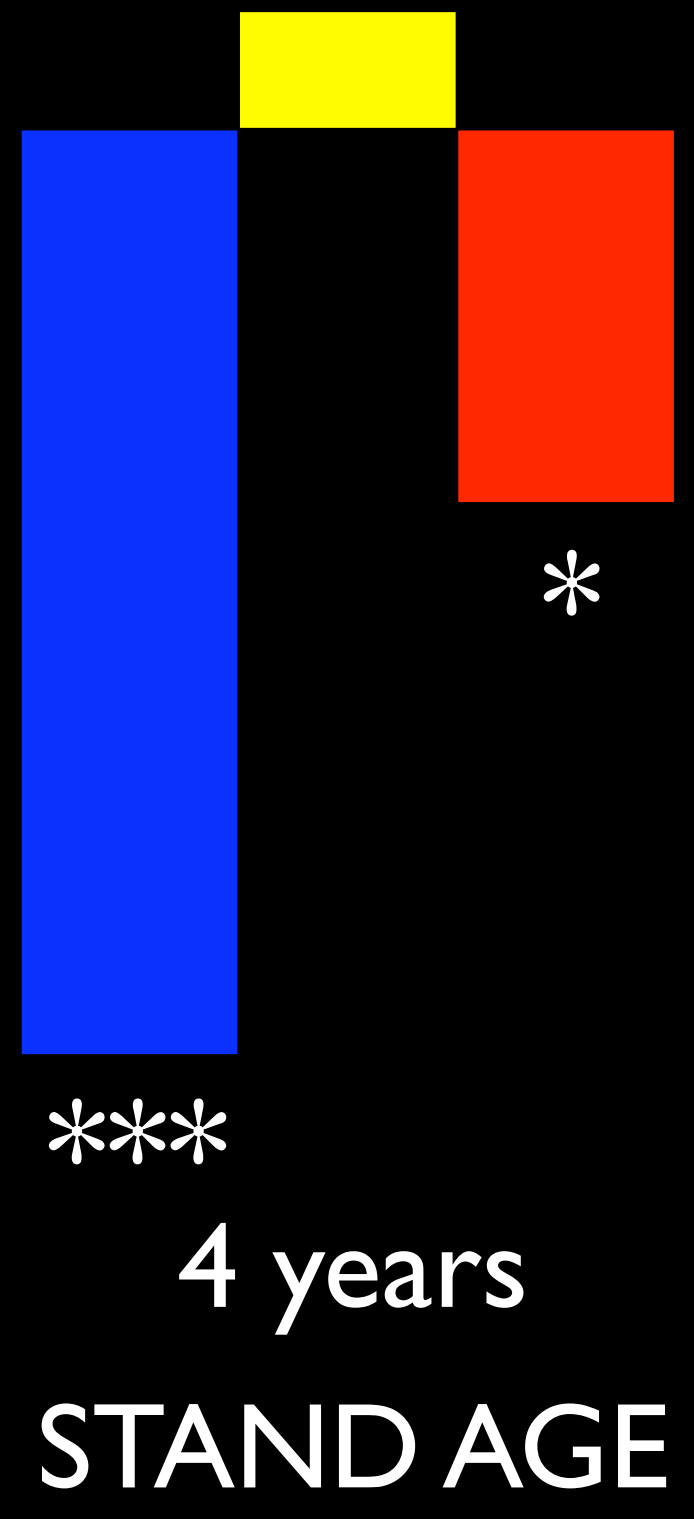

Month

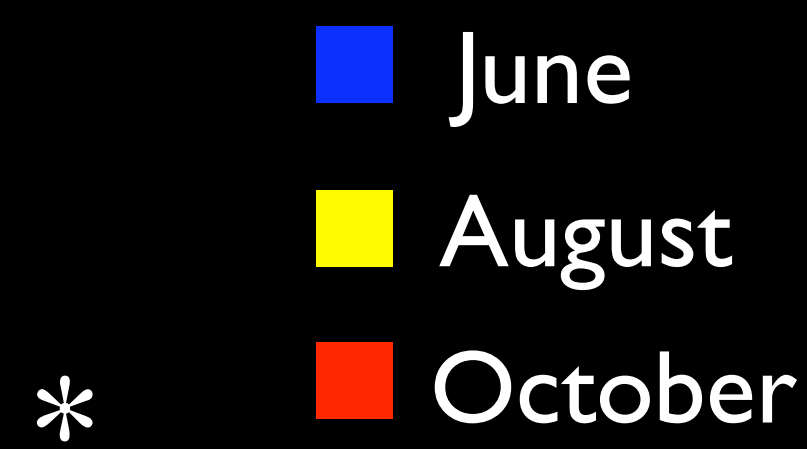




\section{Results}

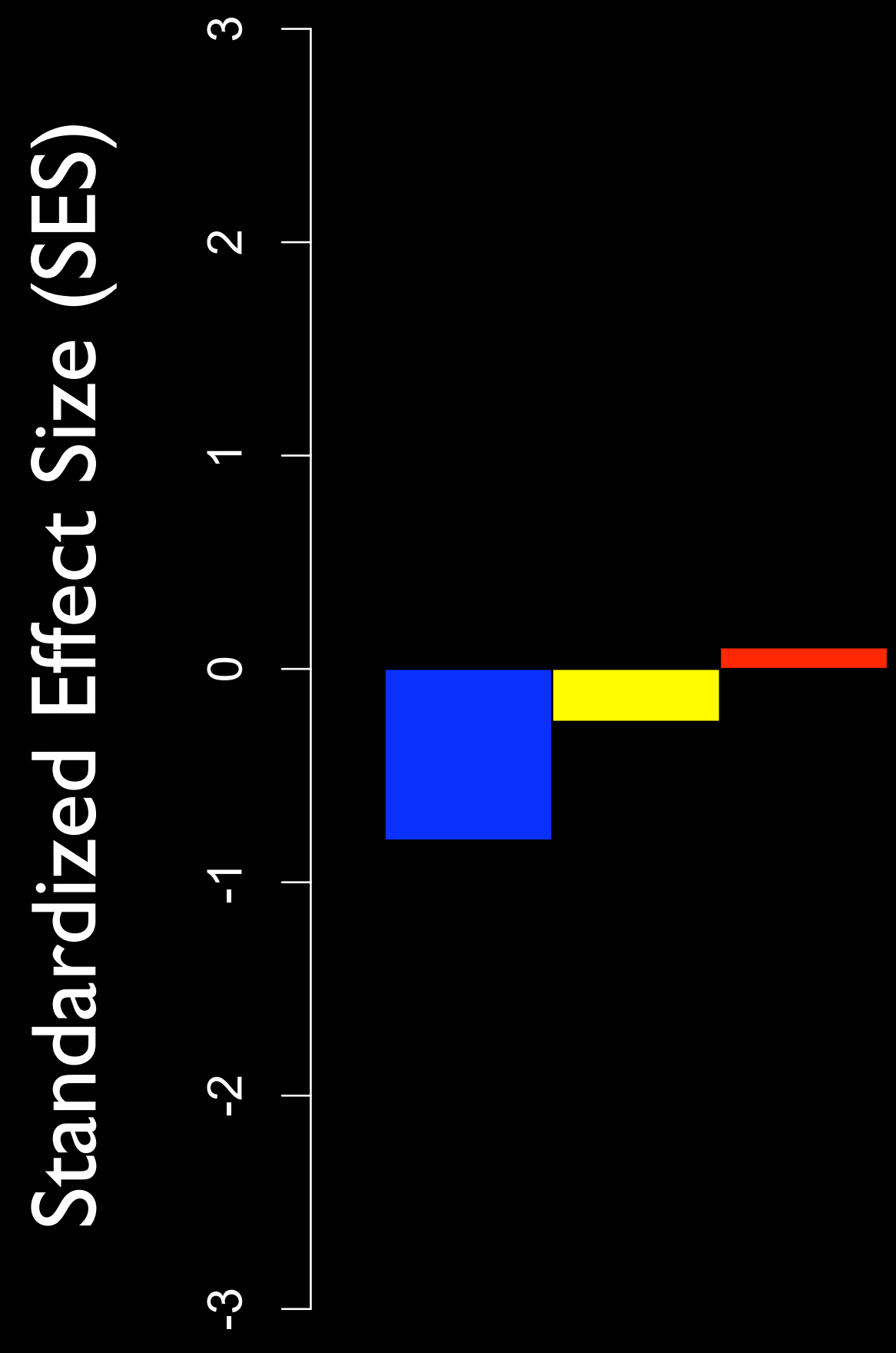

year

$P$-value: $*<0.10, * * *<0.01$

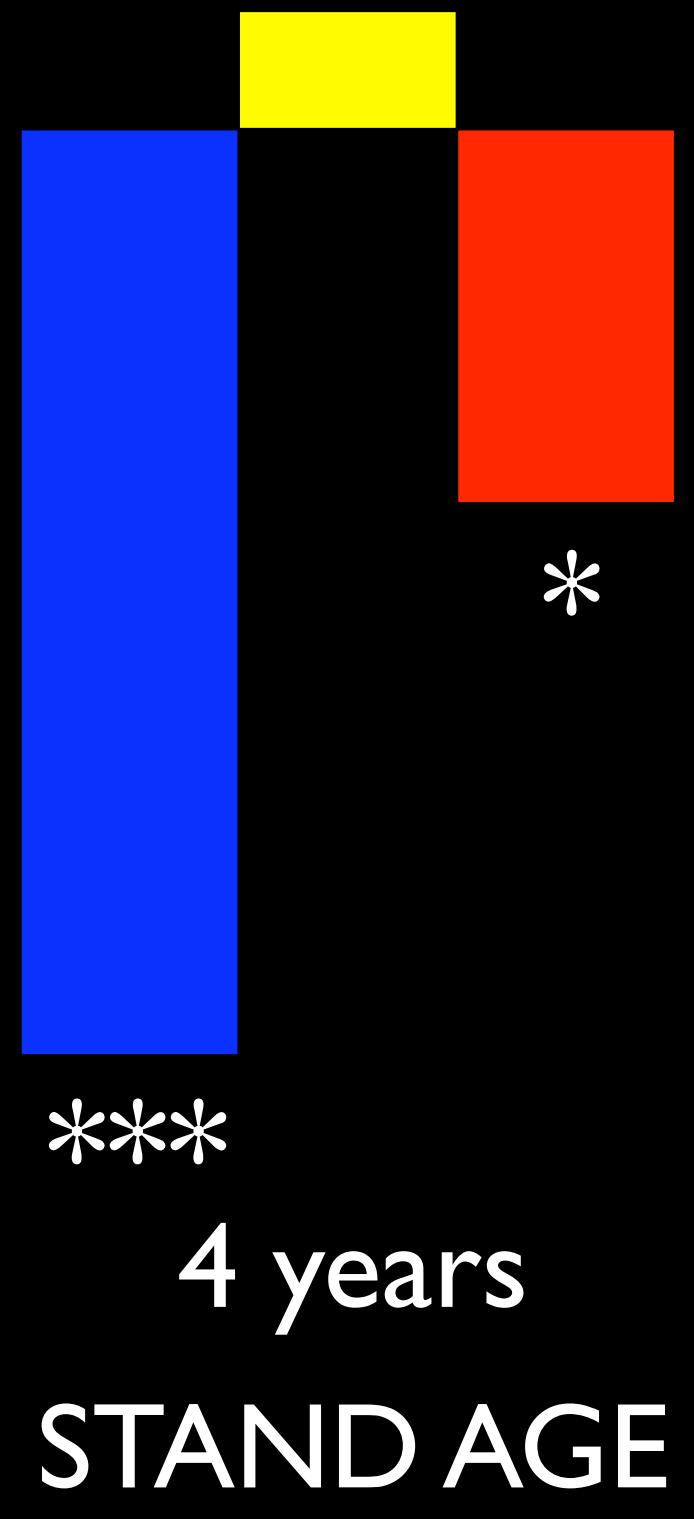

Month

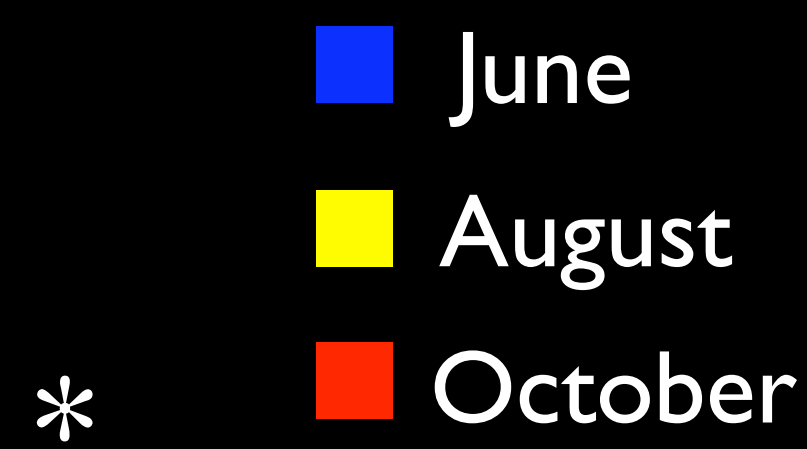


Results

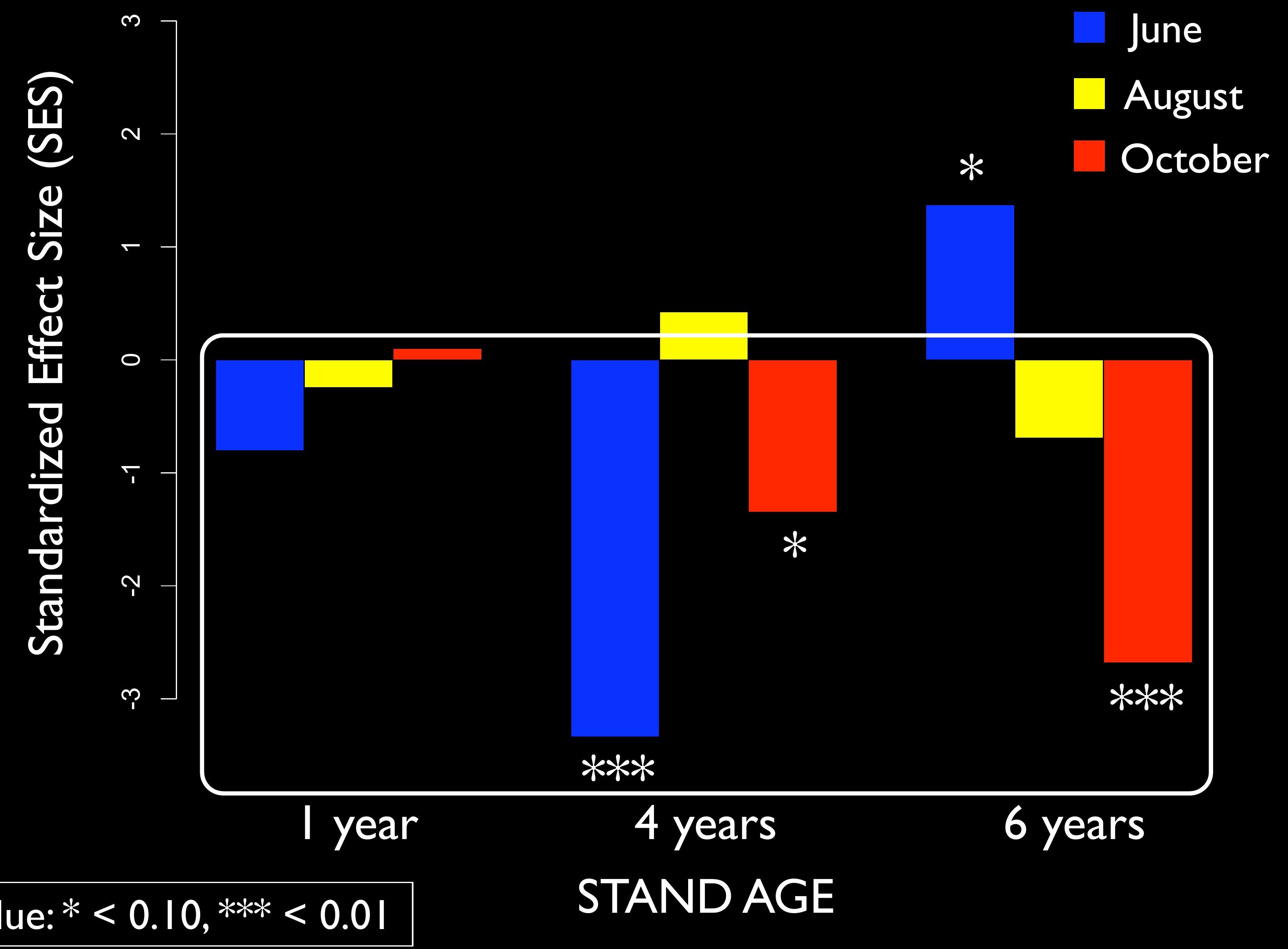


Results

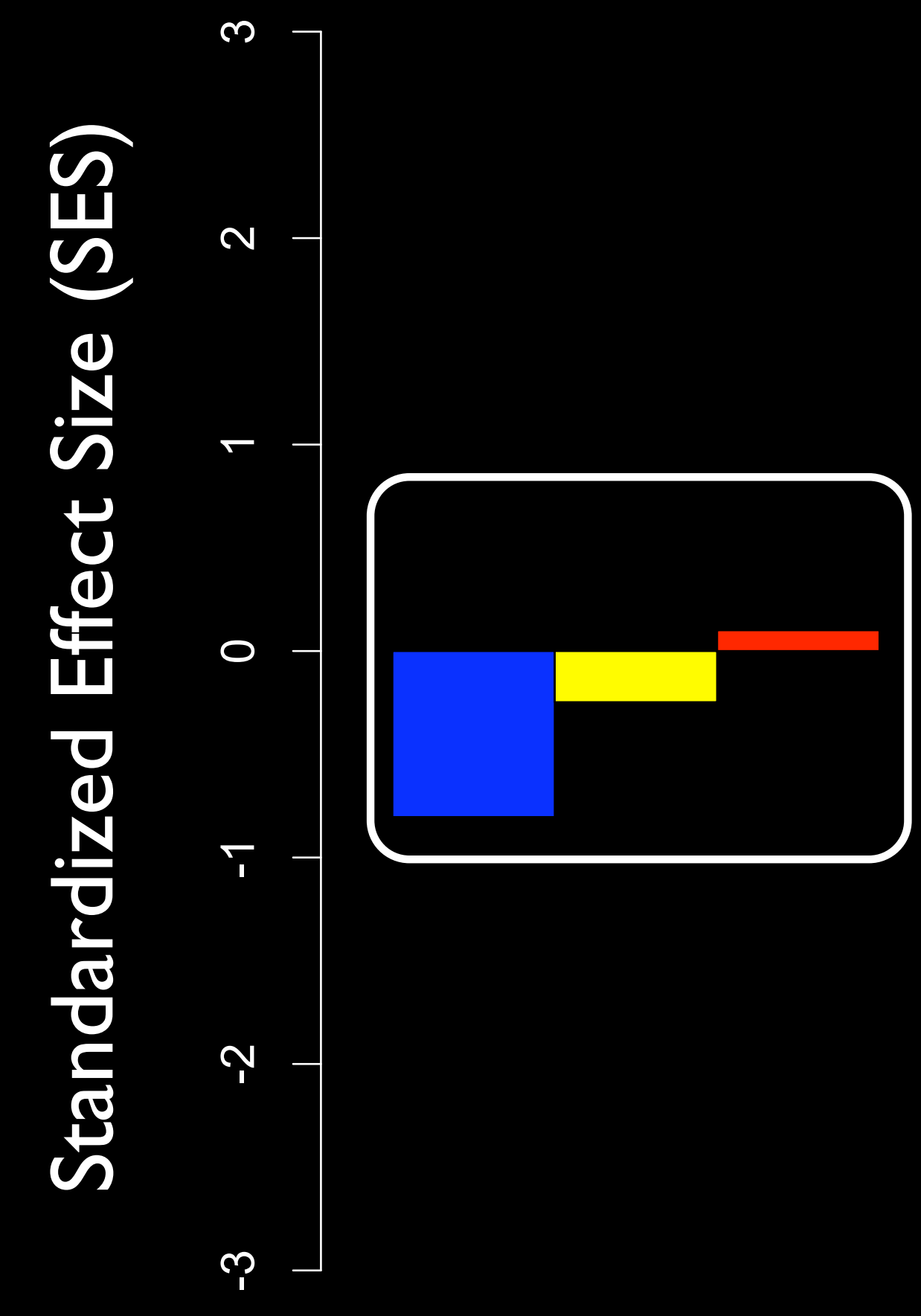

I year

$P$-value: $*<0.10, * * *<0.01$

STAND AGE
Month

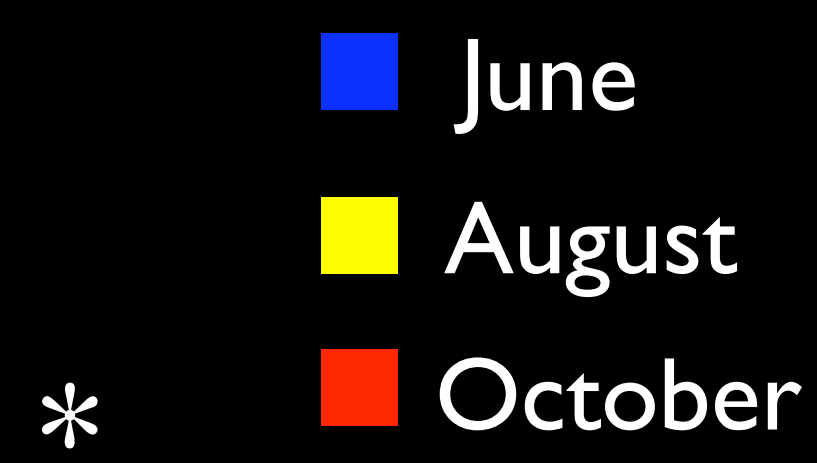

-

4 years

6 years 
Results

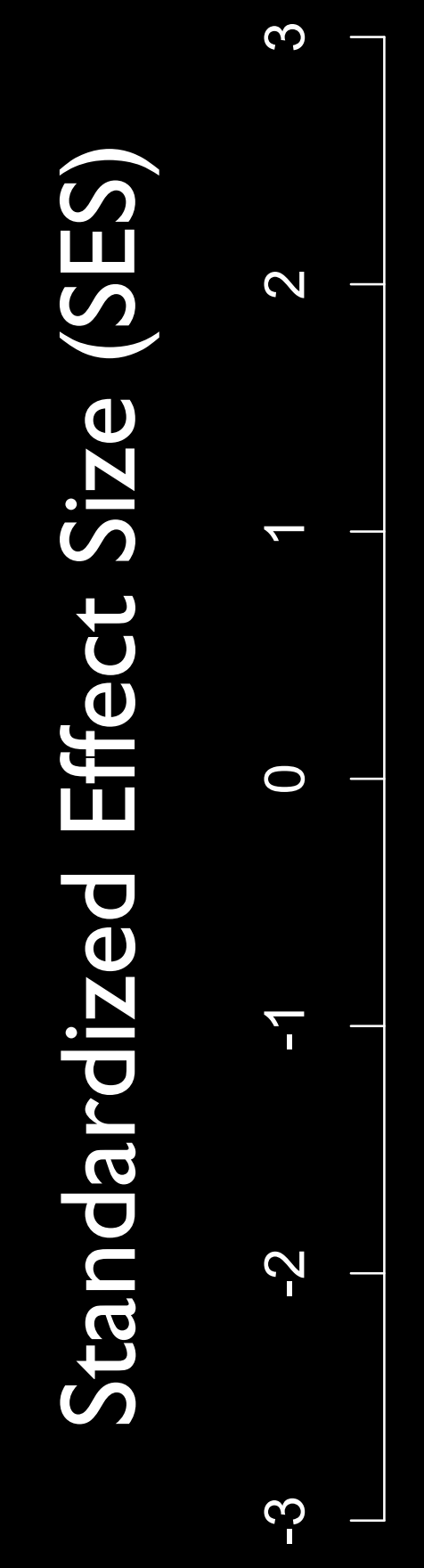

year

$P$-value: $*<0.10, * * *<0.01$ STAND AGE

\section{Month}
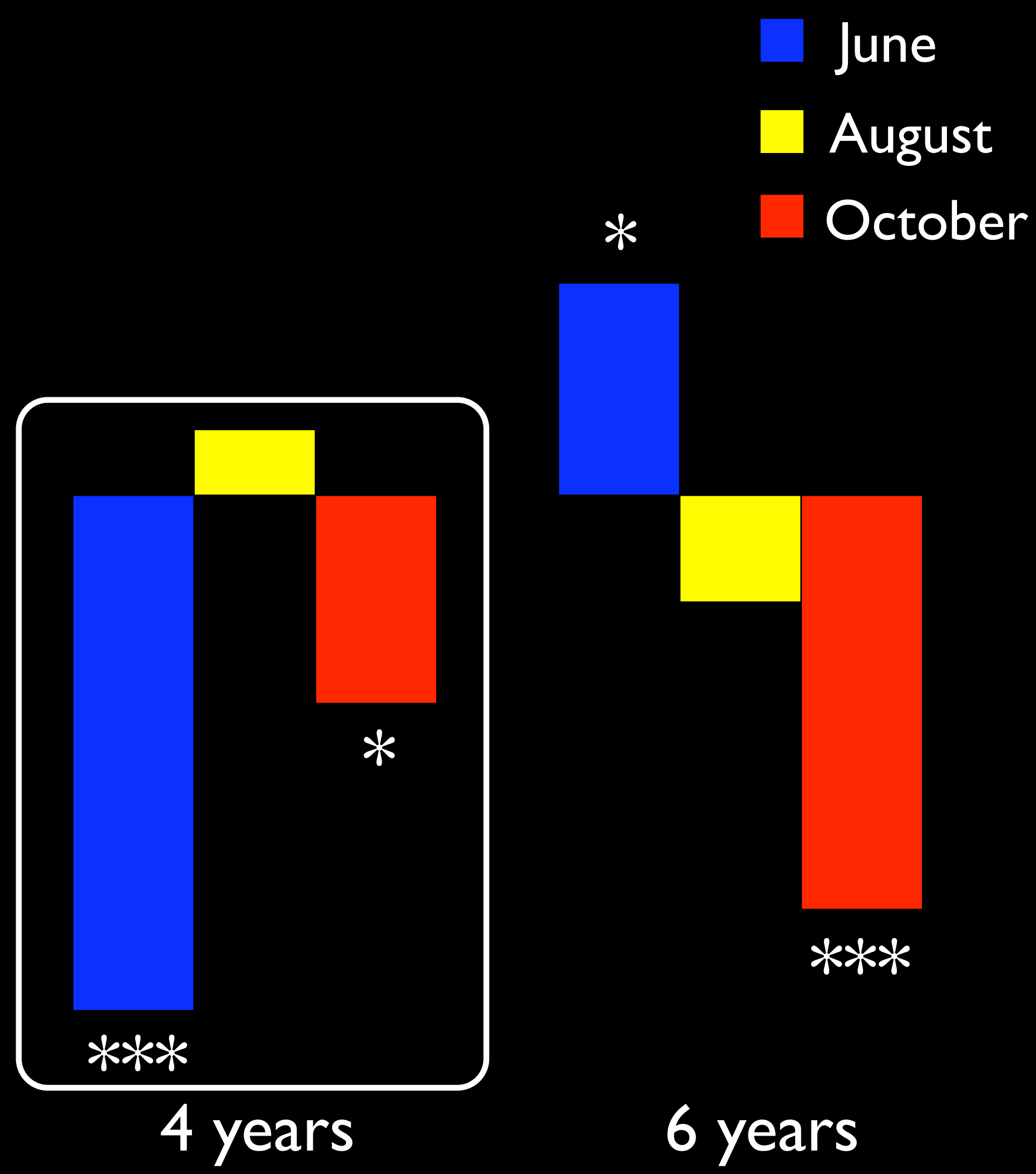

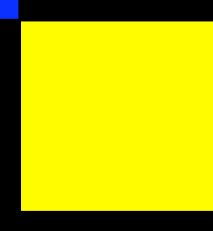

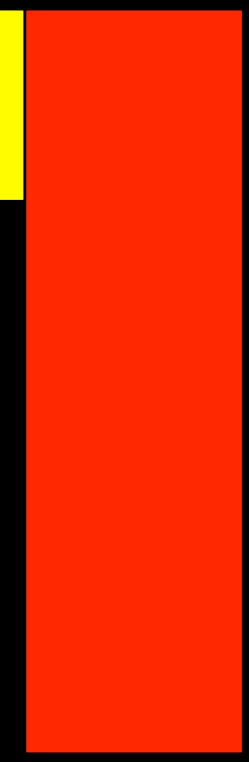
***

6 years 


\section{Results}

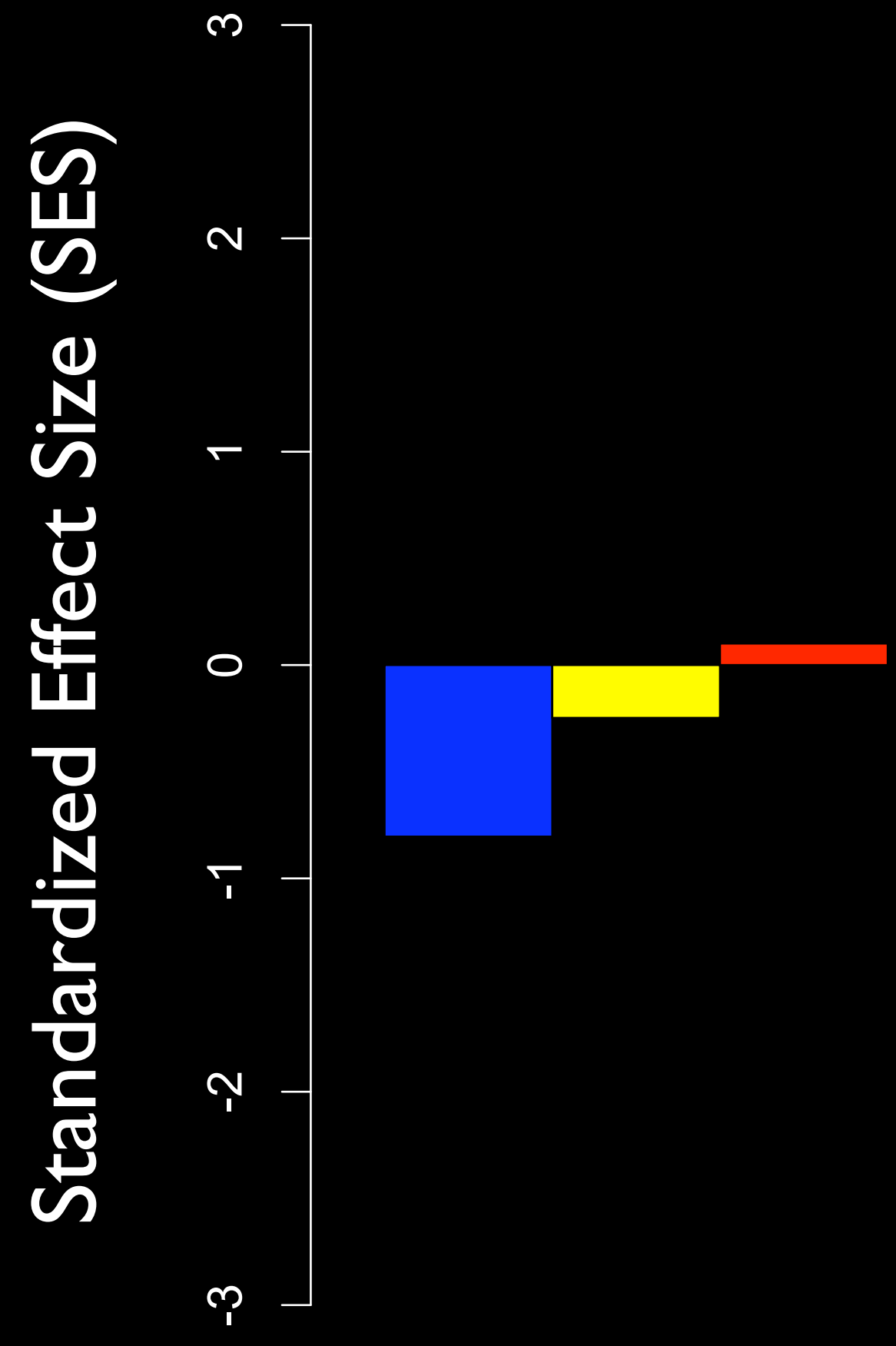

year

$P$-value: $*<0.10, * * *<0.01$

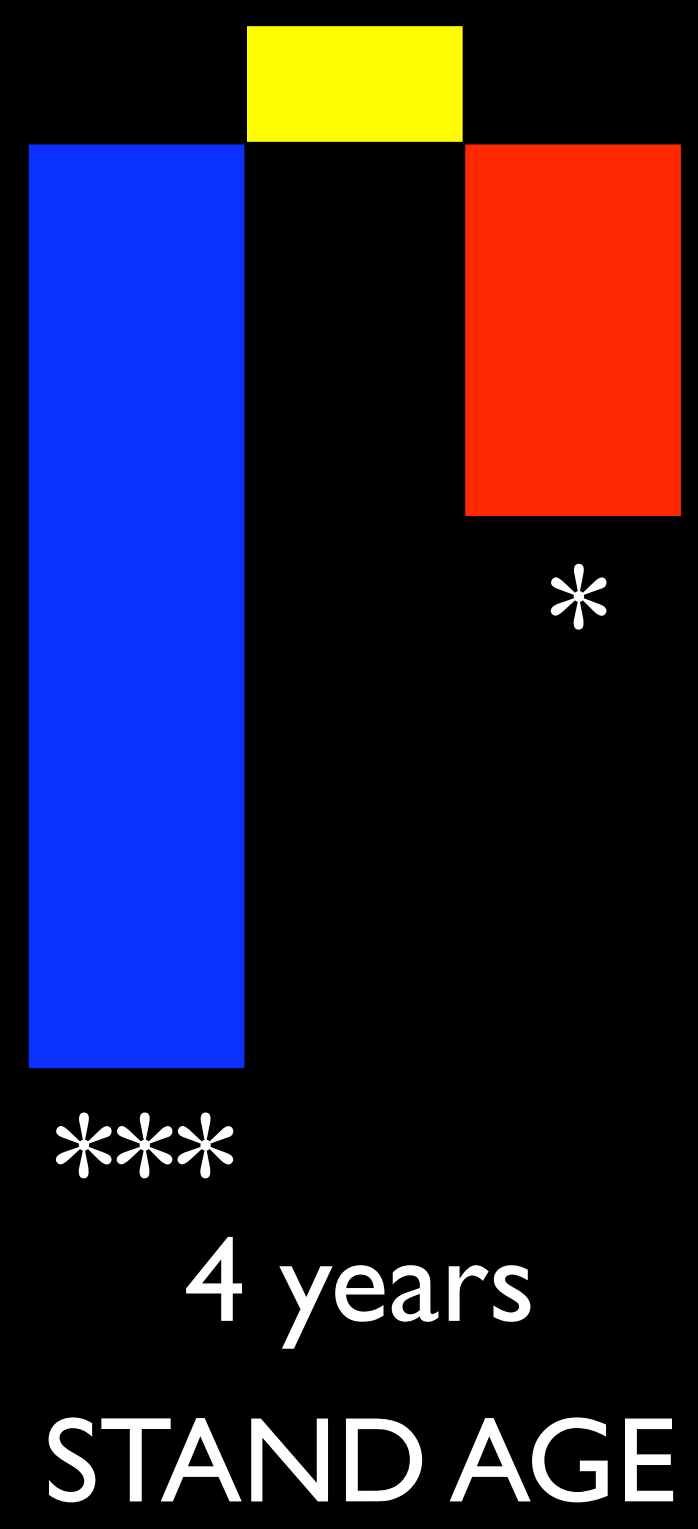

Month

June

August

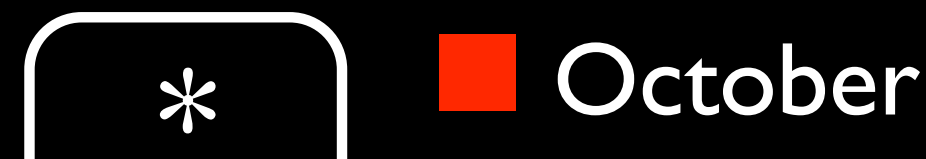

***

6 years

STAND AGE 


\section{Results}
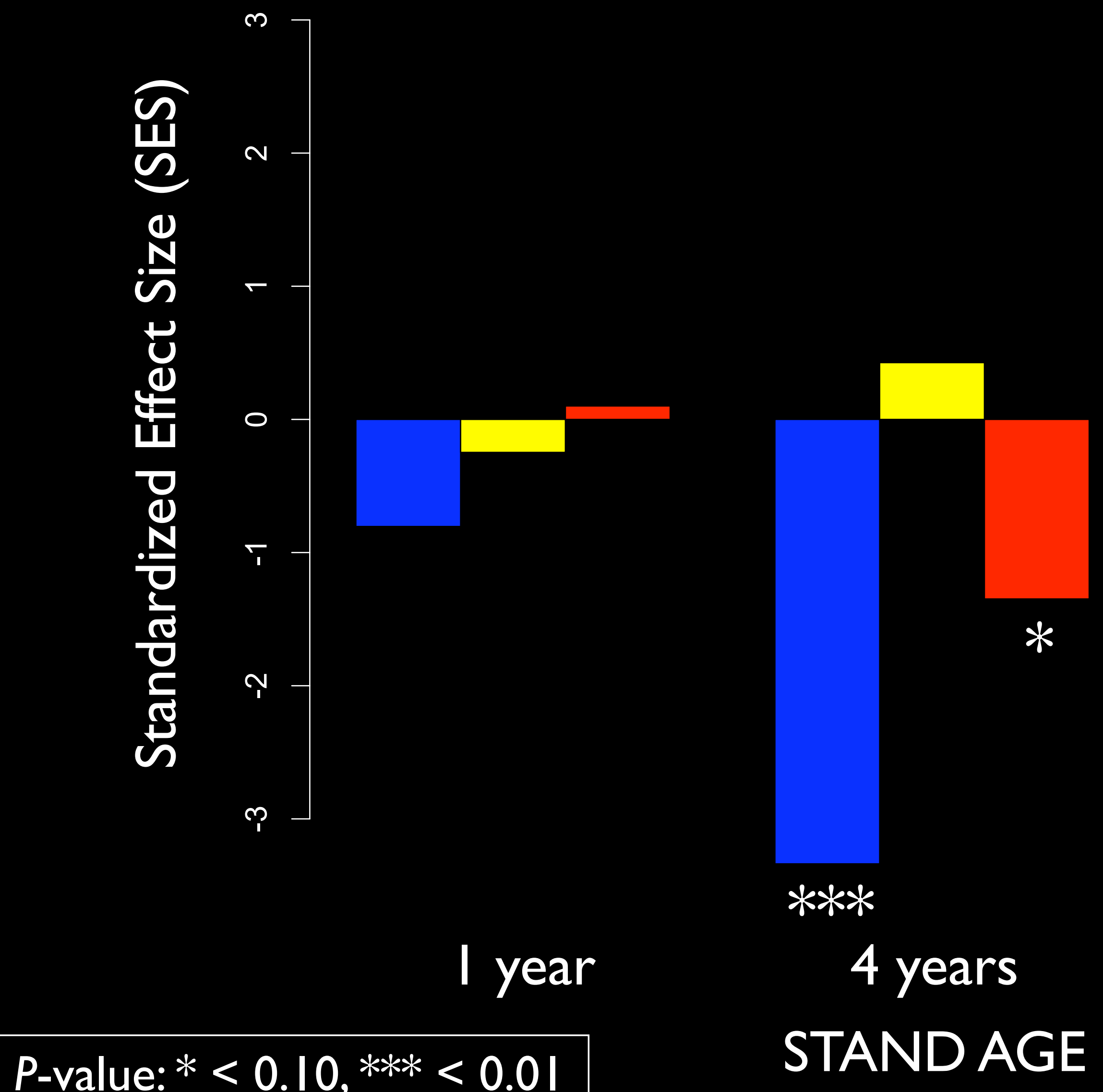

Month

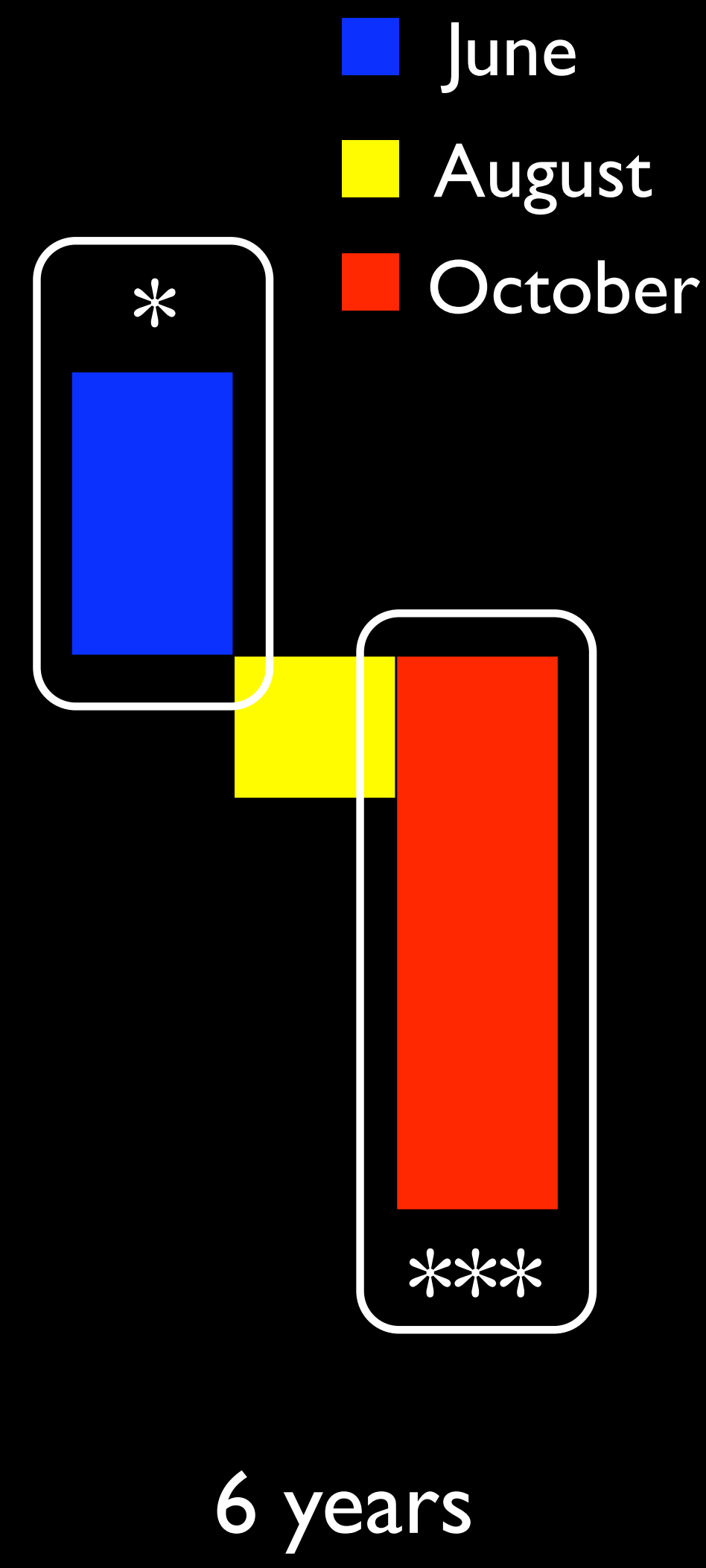

$P$-value: $*<0.10, * * *<0.01$

STAND AGE 


\section{Results}

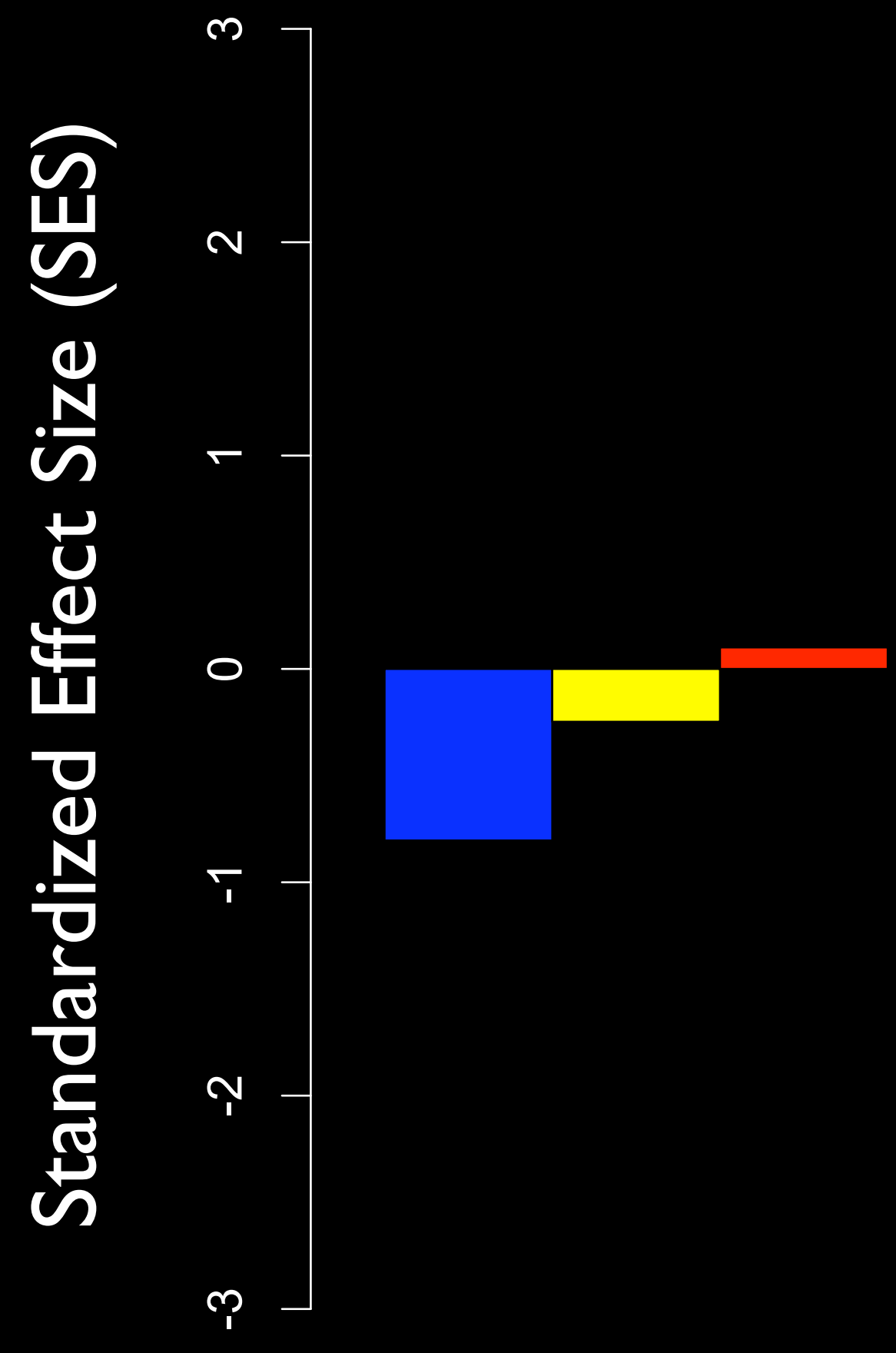

year

$P$-value: $*<0.10, * * *<0.01$

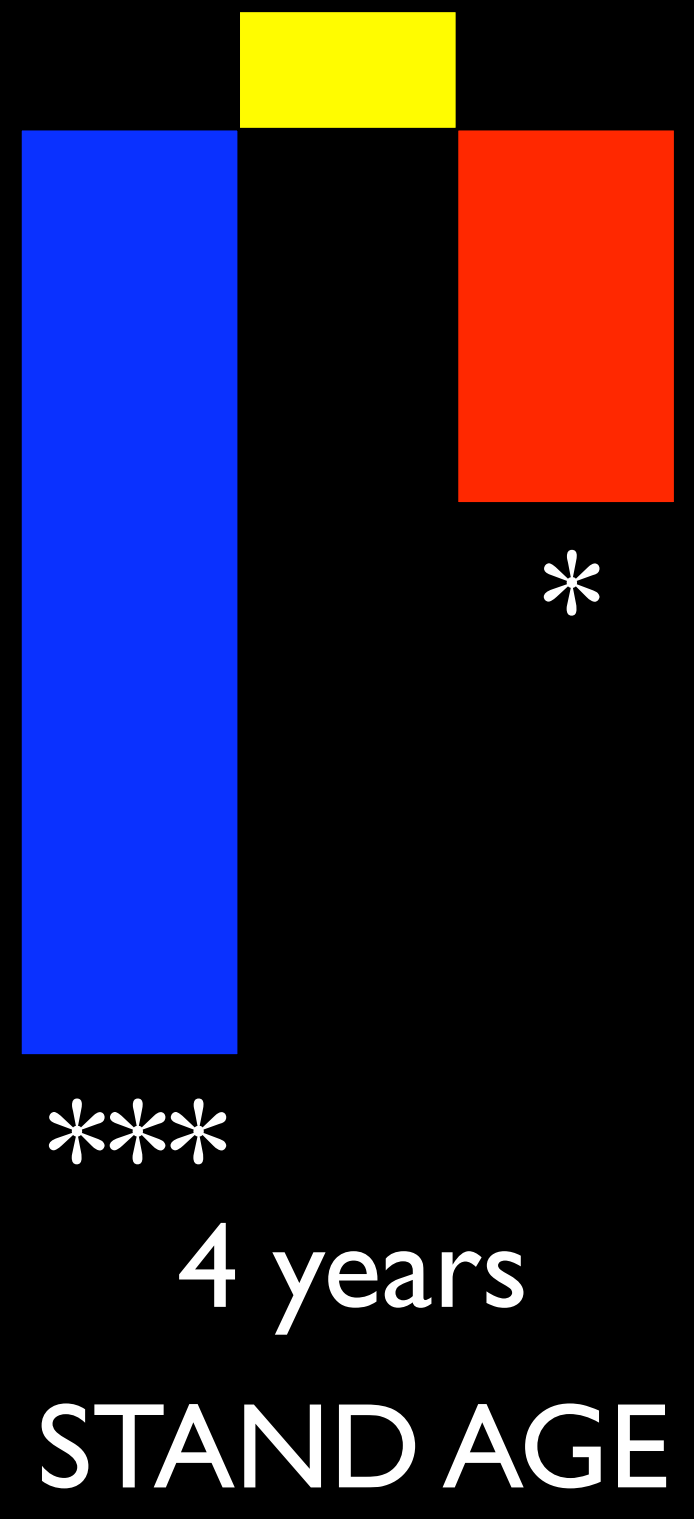

Month

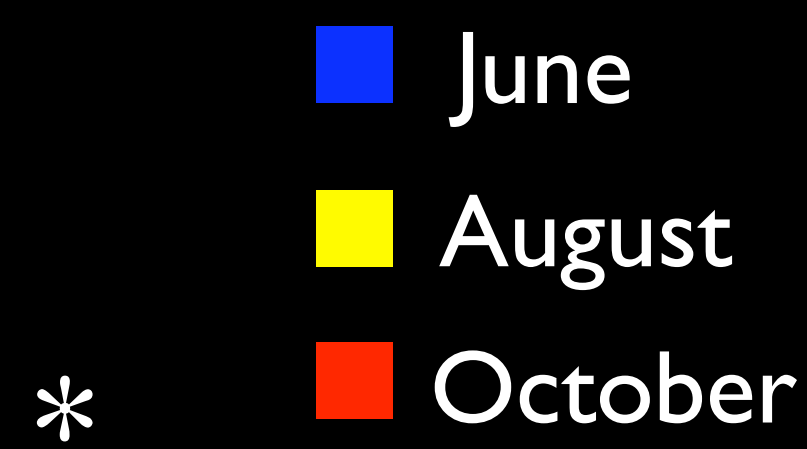




\section{Results}




\section{Results}

- Richness and diversity consistent from stand to stand and month to month 


\section{Results}

- Richness and diversity consistent from stand to stand and month to month

- Community composition varied by month and stand 


\section{Results}

- Richness and diversity consistent from stand to stand and month to month

- Community composition varied by month and stand

\section{PerMANOVA:}

month*stand $P<0.001 * * *$ 


\section{October}

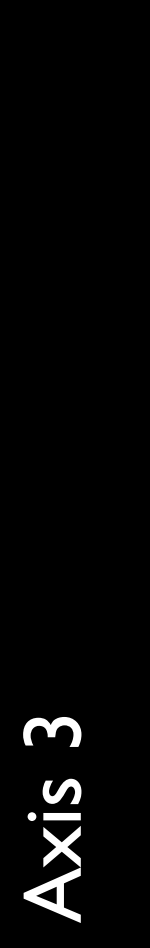

PerMANOVA:

month*stand $\mathrm{P}<0.001$ ****
Stand Age

$\checkmark$ I year

$\Delta 4$ years

06 years

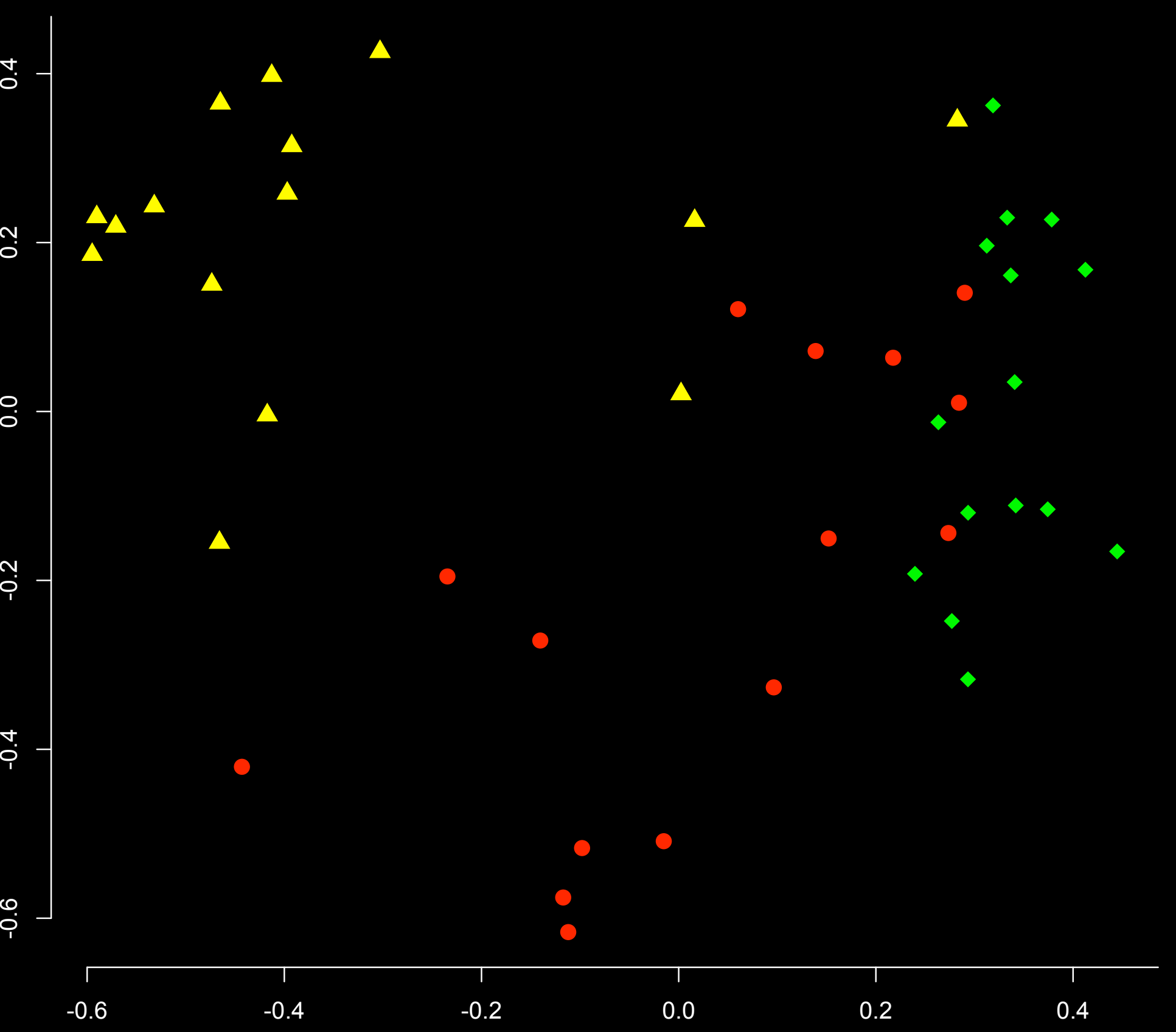

Axis I 


\section{October}

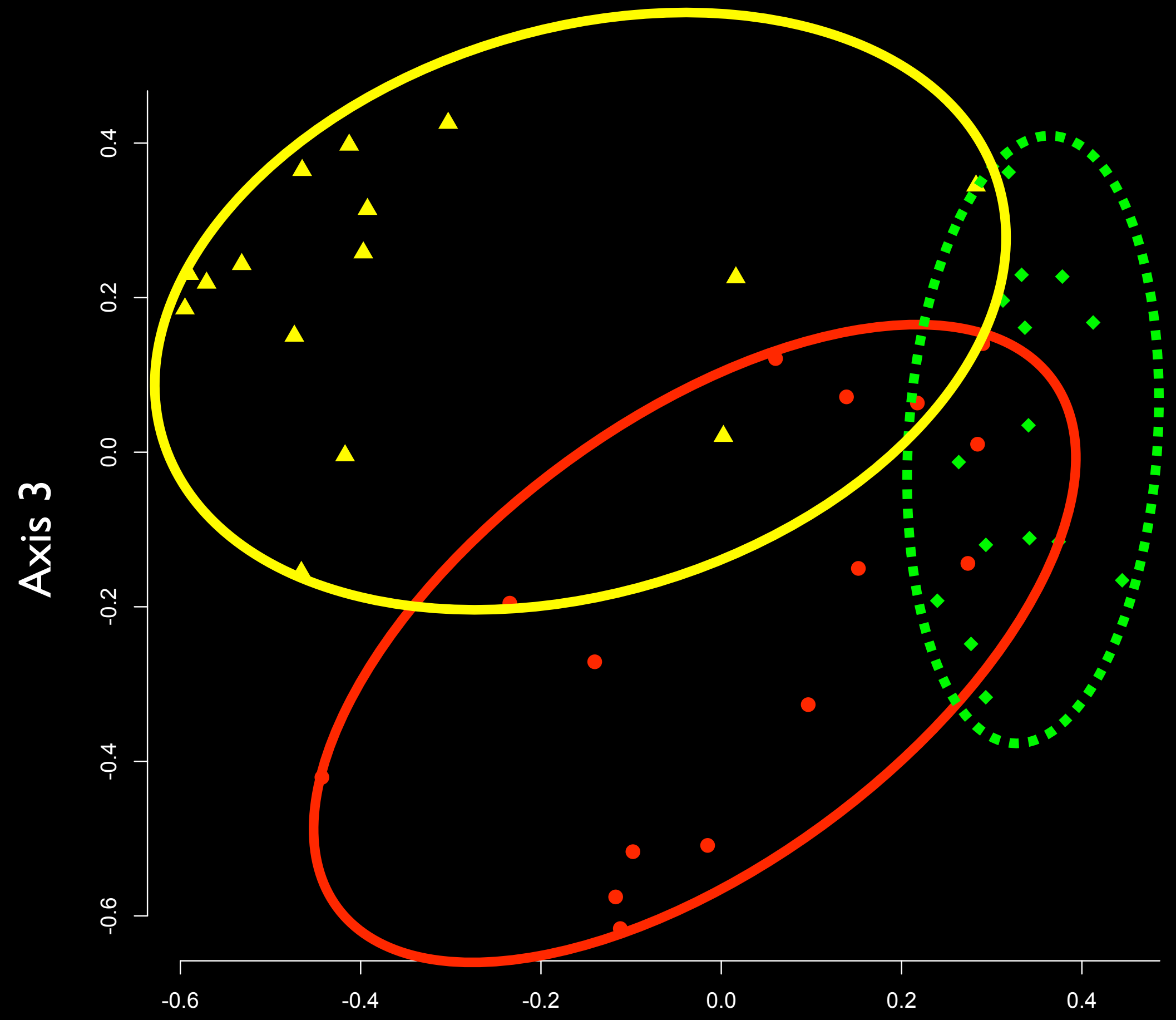

Axis I
Stand Age

$\checkmark$ I year

$\Delta 4$ years

06 years
PerMANOVA:

month*stand $\mathrm{P}<0.001$ **** 
Month

\author{
June
}

August

October

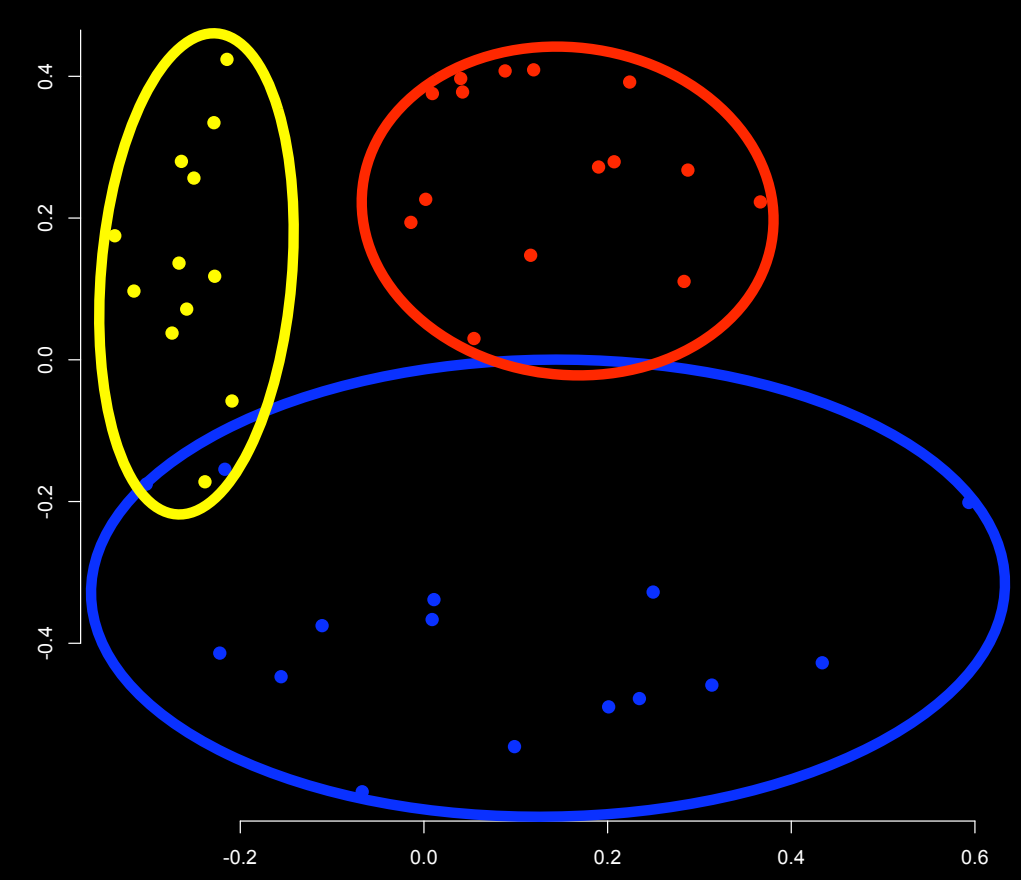

2005 (I year)

\author{
PerMANOVA:
}

month*stand $\mathrm{P}<0.001$ * *** 
Month

June

$\square$ August

$\square$ October

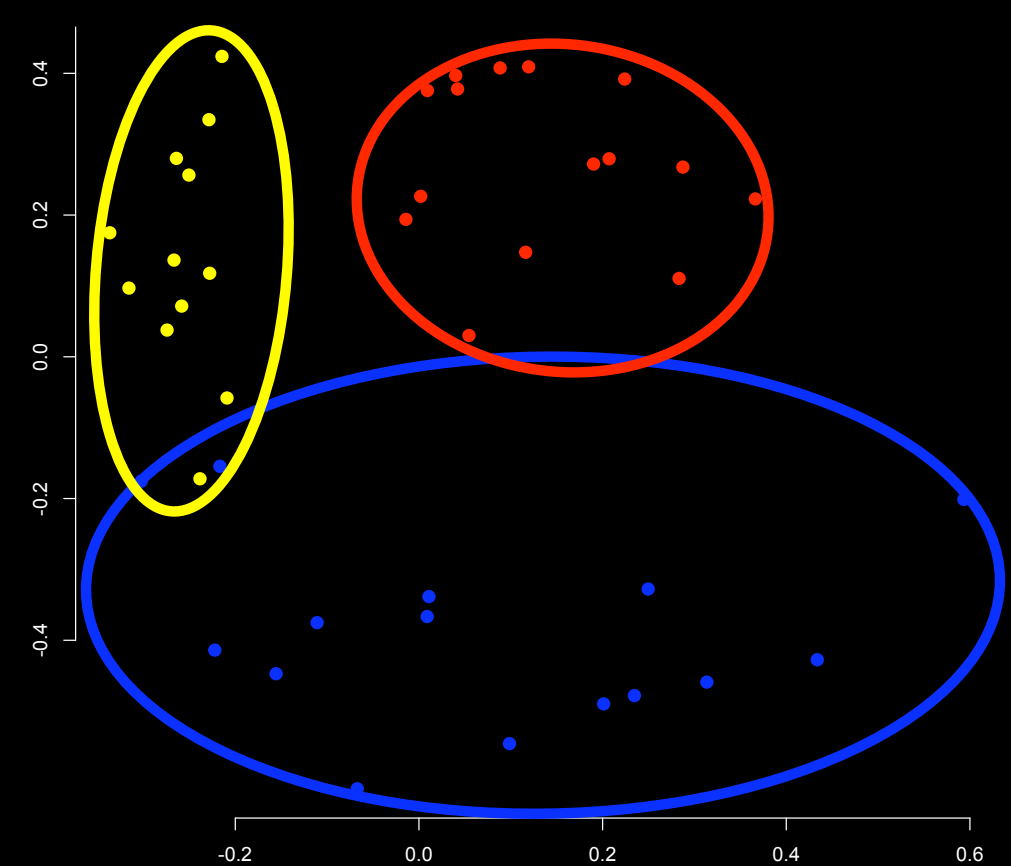

2005 (I year)

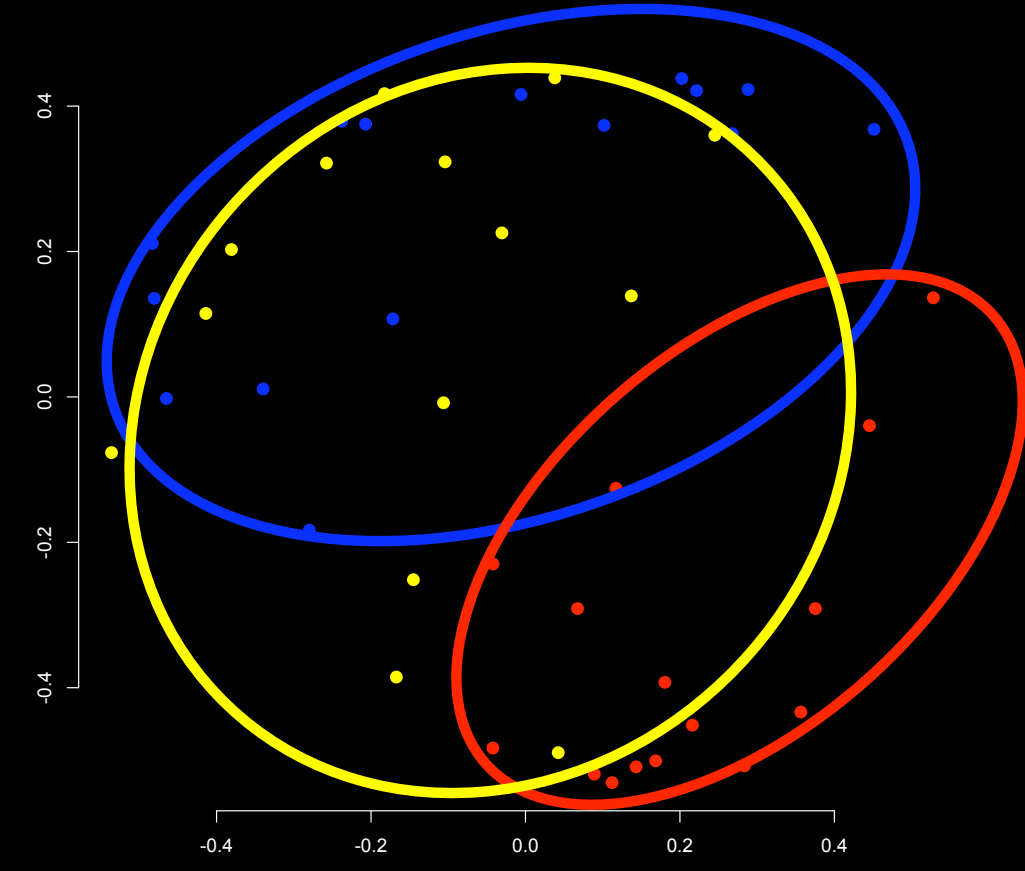

2002 (4 years)

\section{PerMANOVA:}

month*stand $\mathrm{P}<0.001 * * *$ 
Month

$\square$ June

$\square$ August

October

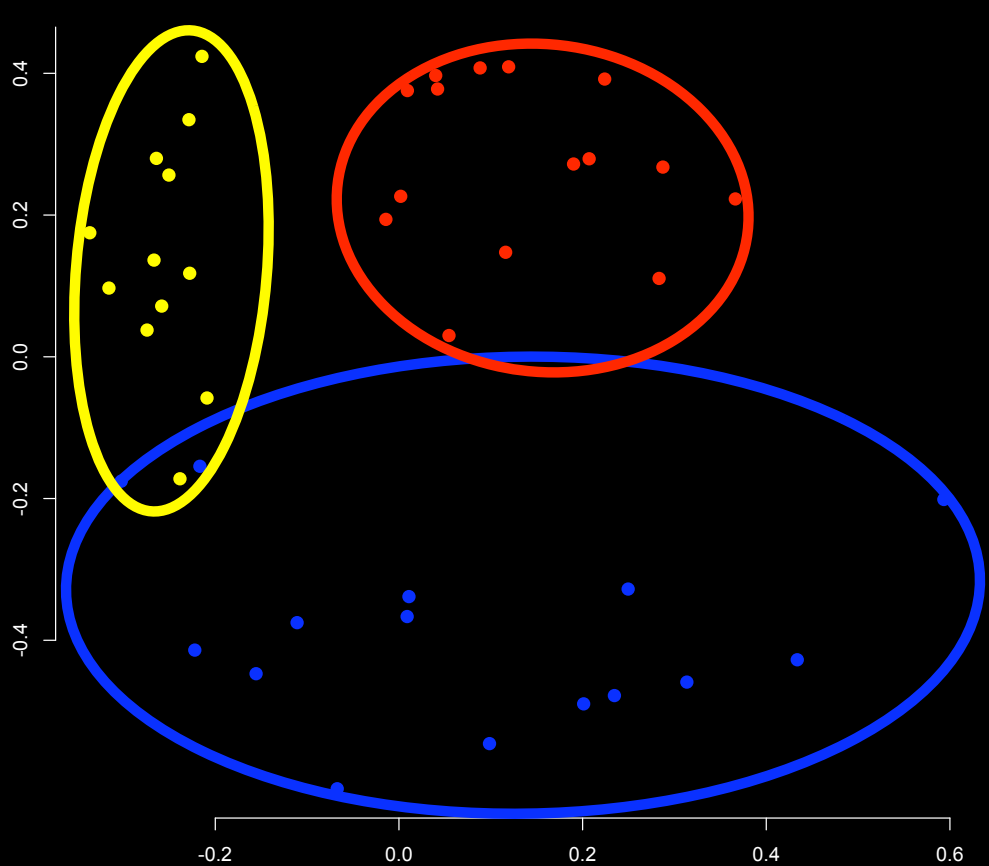

2005 (I year)

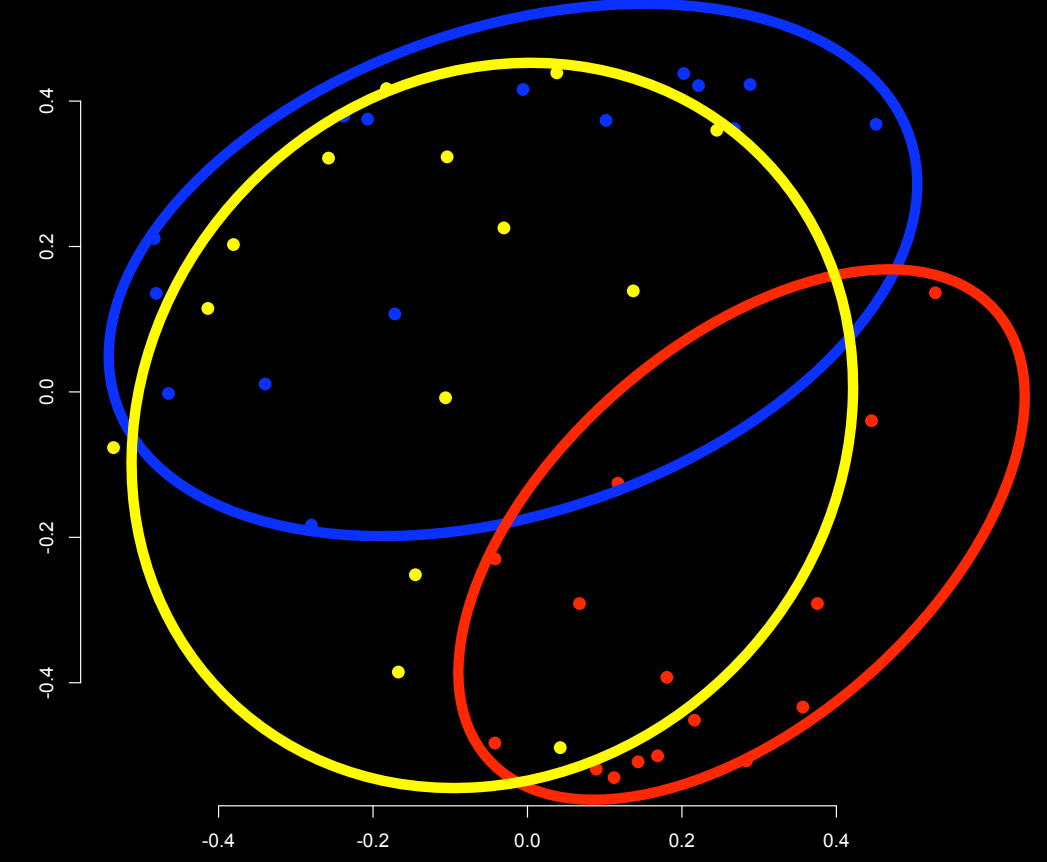

2002 (4 years)

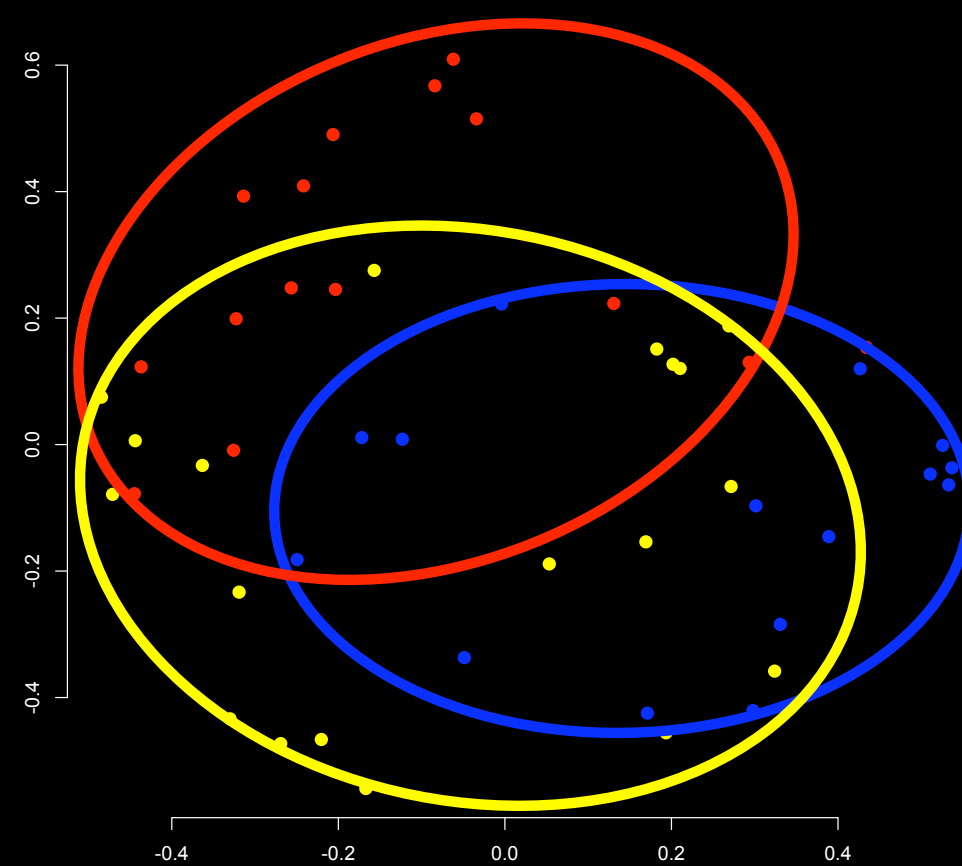

2000 (6 years)

PerMANOVA:

month*stand $\mathrm{P}<0.001 * * * *$ 
Month

$\square$ June

$\square$ August

October

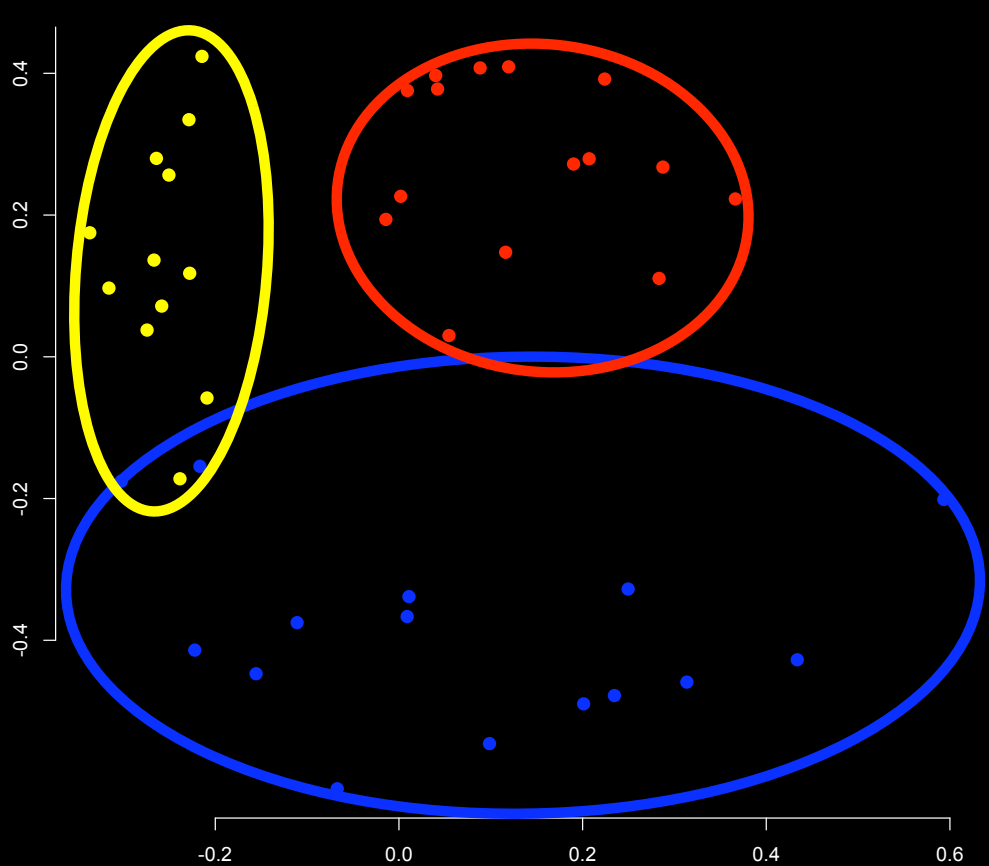

2005 (I year)

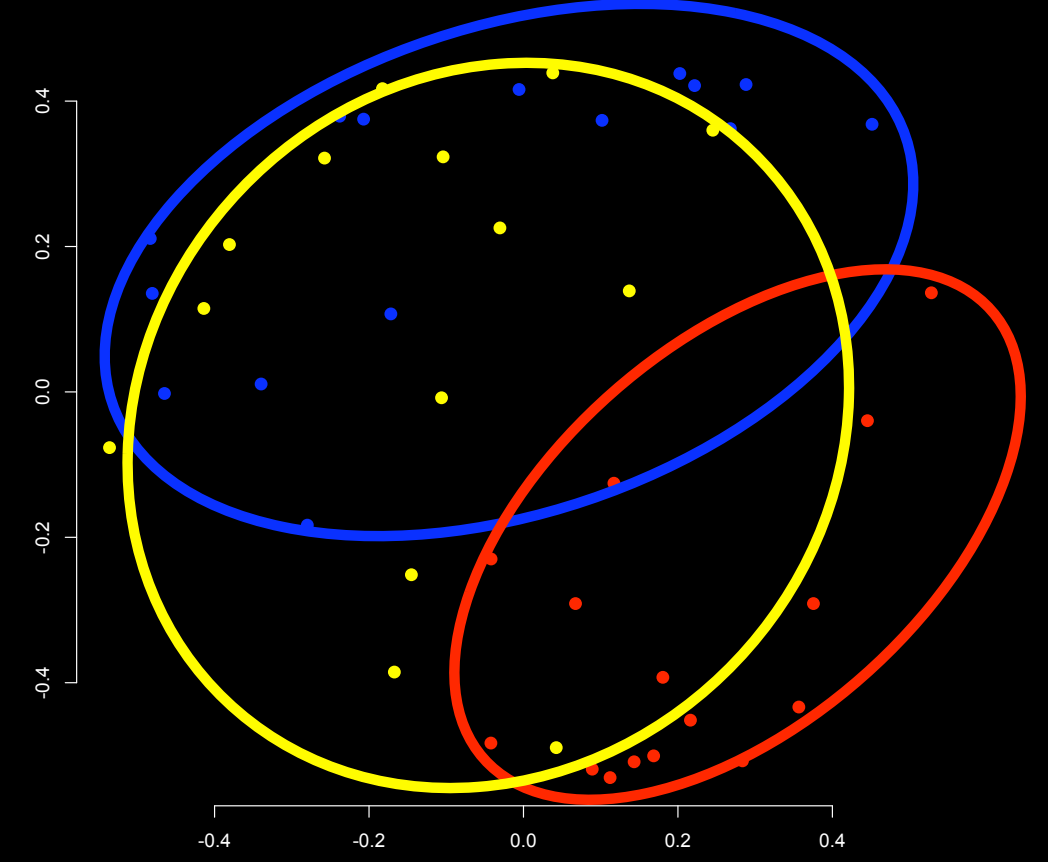

2002 (4 years)

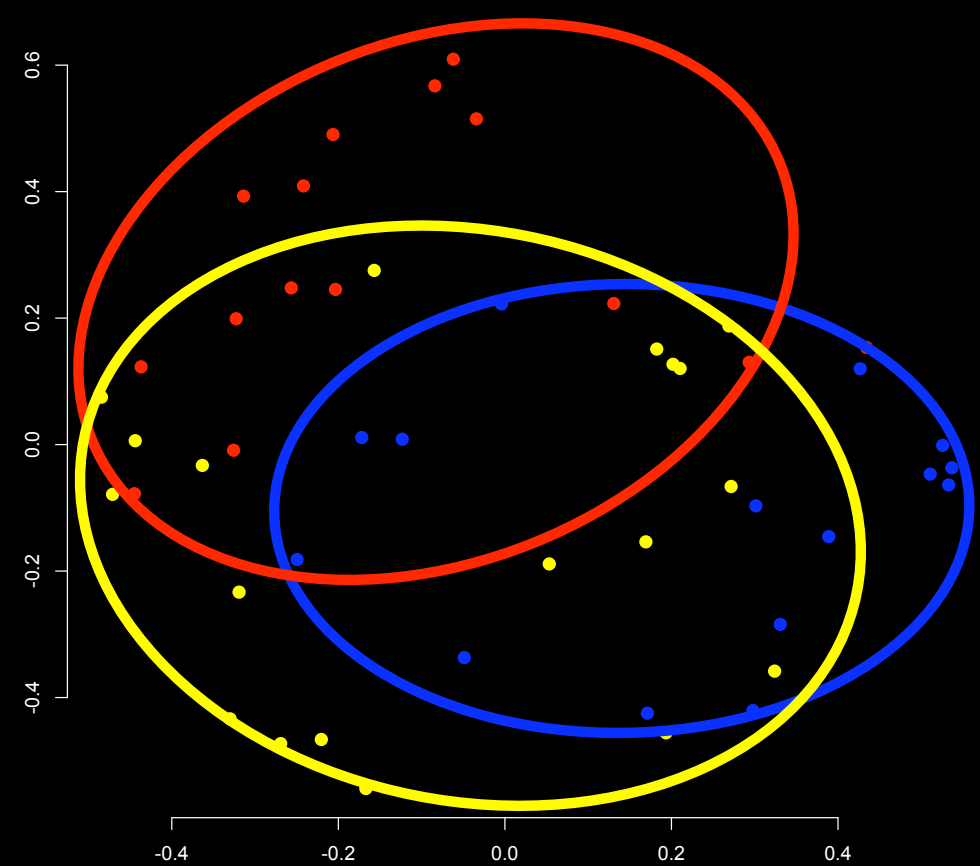

2000 (6 years)

Stand Age

PerMANOVA:

month*stand $\mathrm{P}<0.001 * * *$ 


\section{Conclusions}




\section{Conclusions}

- Local interactions can play a role in producing local community structure 


\section{Conclusions}

- Local interactions can play a role in producing local community structure

- Forces vary by month and by stand 


\section{Conclusions}

- Local interactions can play a role in producing local community structure

- Forces vary by month and by stand

- Could result from stand-environmental or compositional differences 


\section{Take Home}




\section{Take Home}

- Understanding local processes is important 


\section{Take Home}

- Understanding local processes is important

- Regional process are also important 


\section{Take Home}

- Understanding local processes is important

- Regional process are also important

- Focus on the interplay of ecological and evolutionary process across spatial and temporal scales 


\section{Take Home}
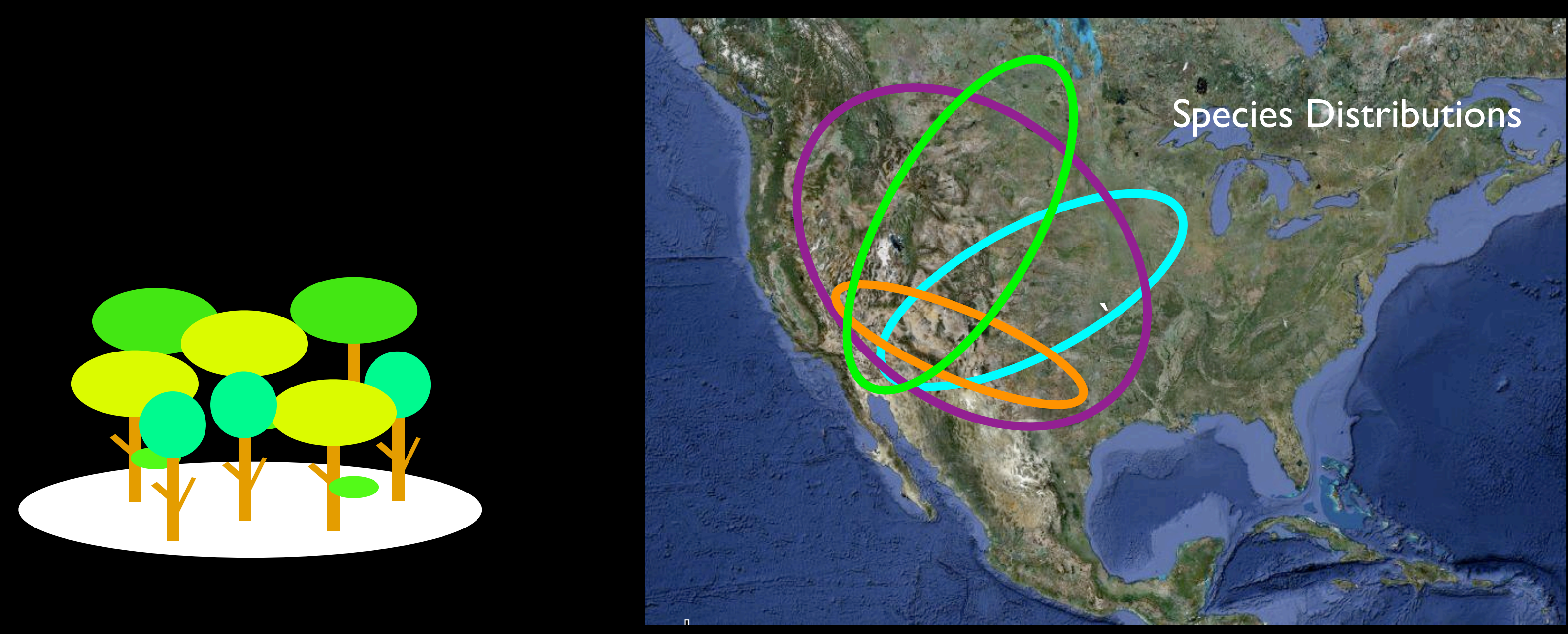

\section{Local Scale}

\section{Larger Scales}




\section{Take Home}

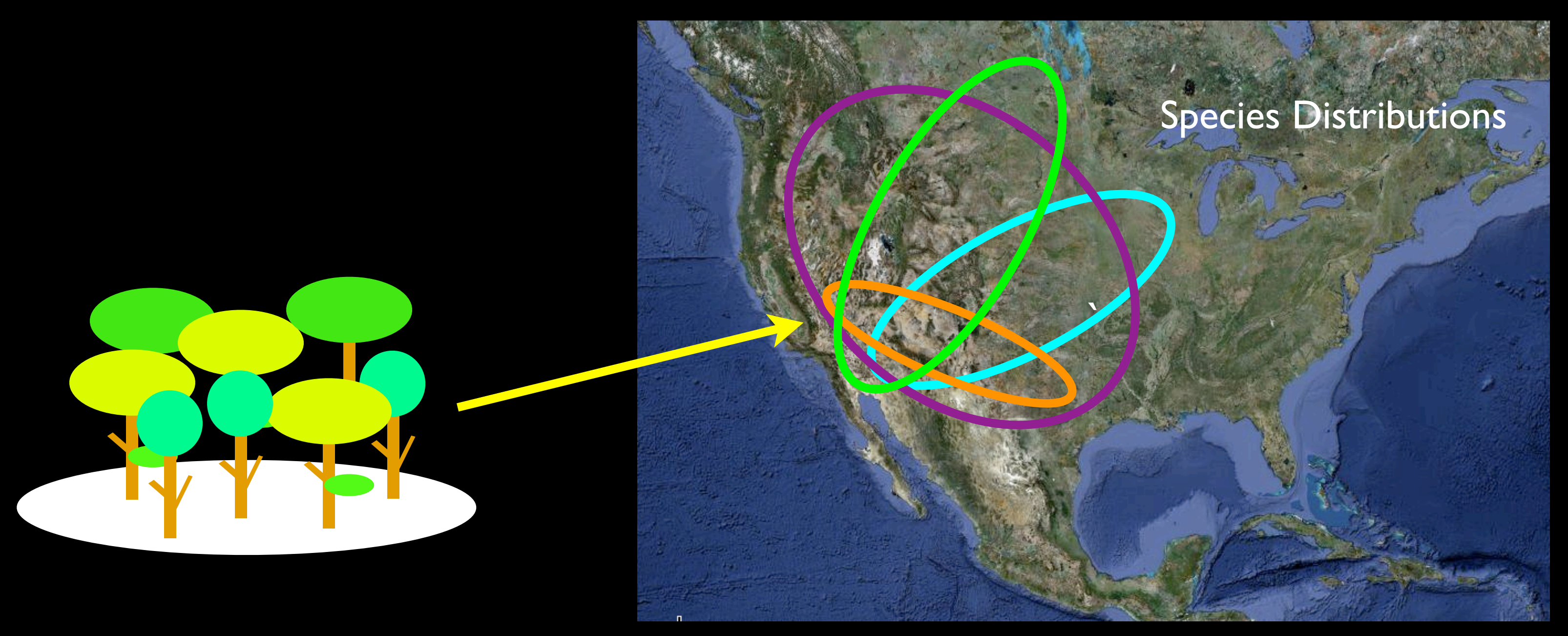

Local Scale

Larger Scales 


\section{Take Home}

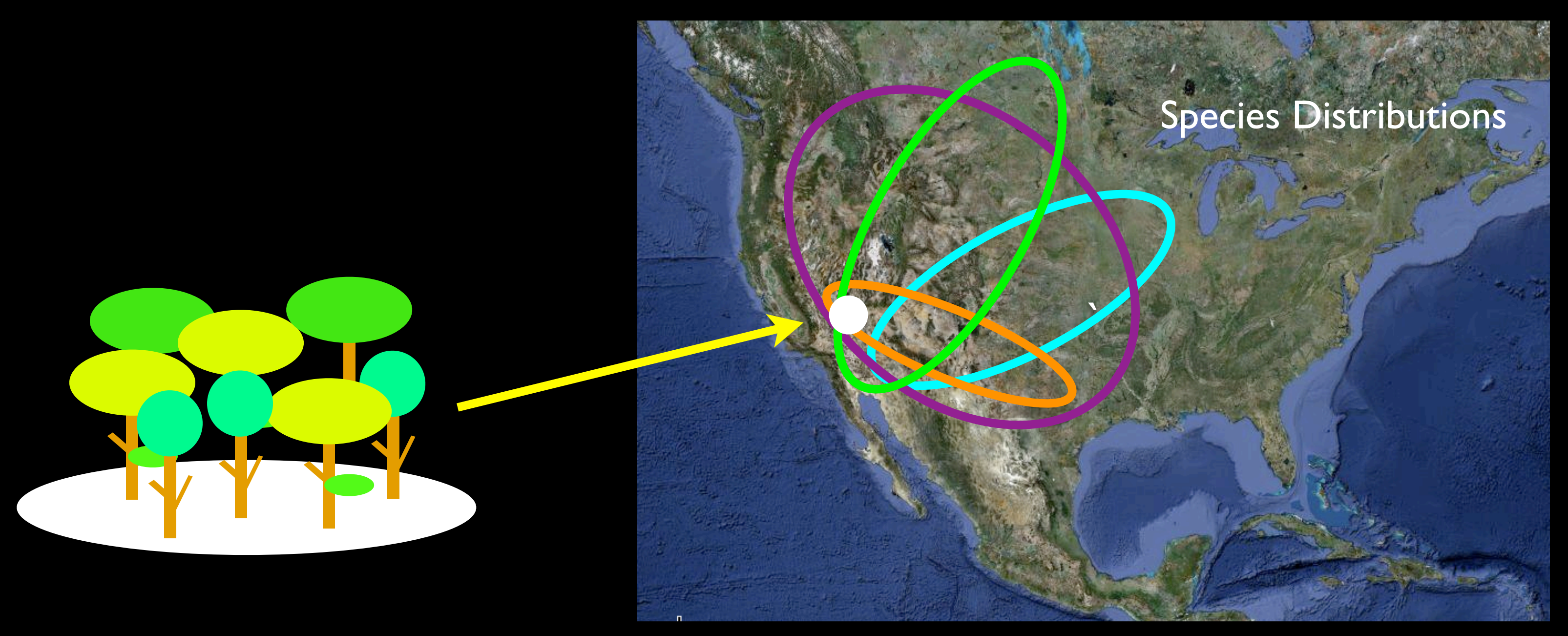

Local Scale

Larger Scales 


\section{Take Home}

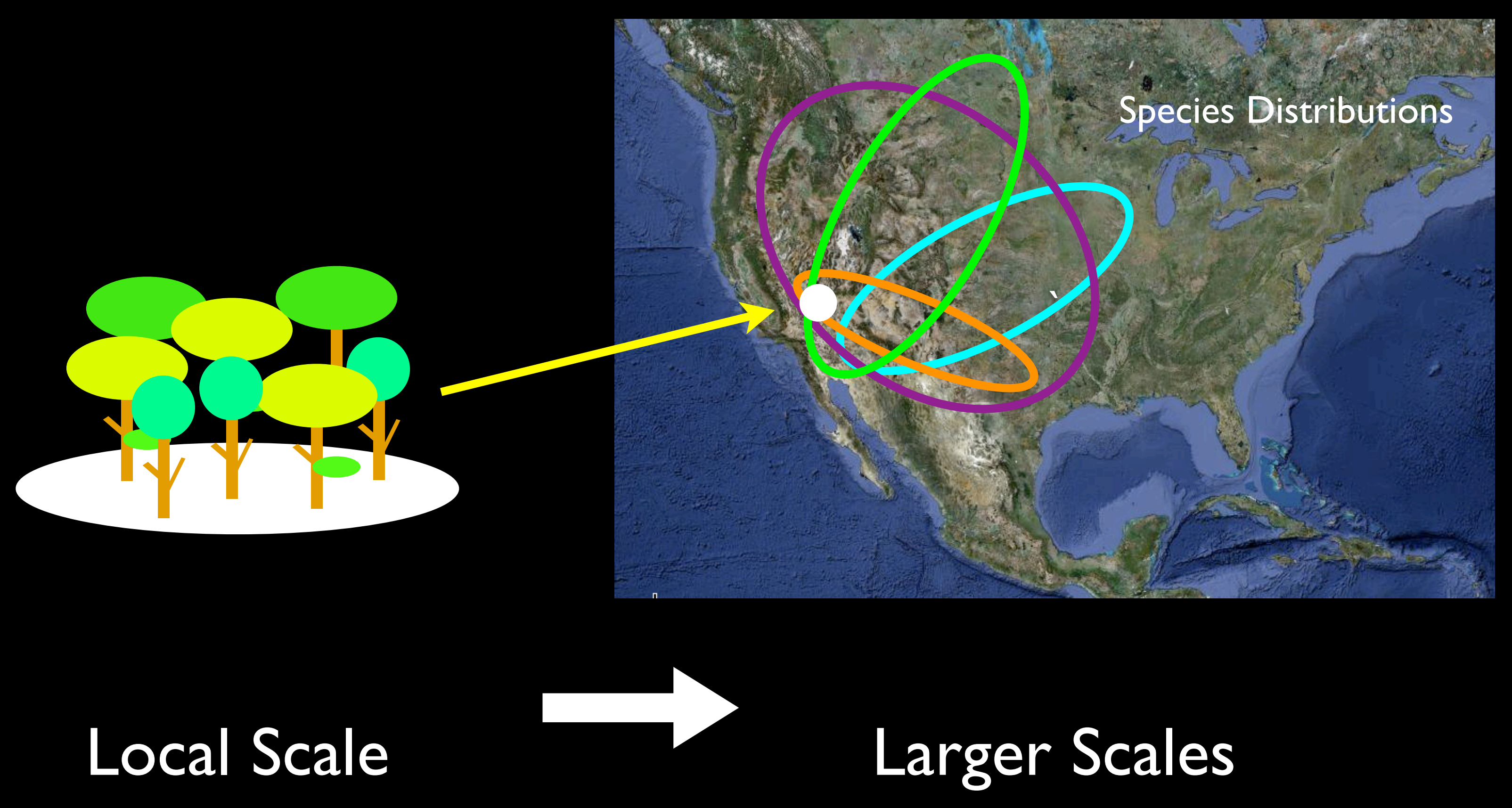




\section{Take Home}

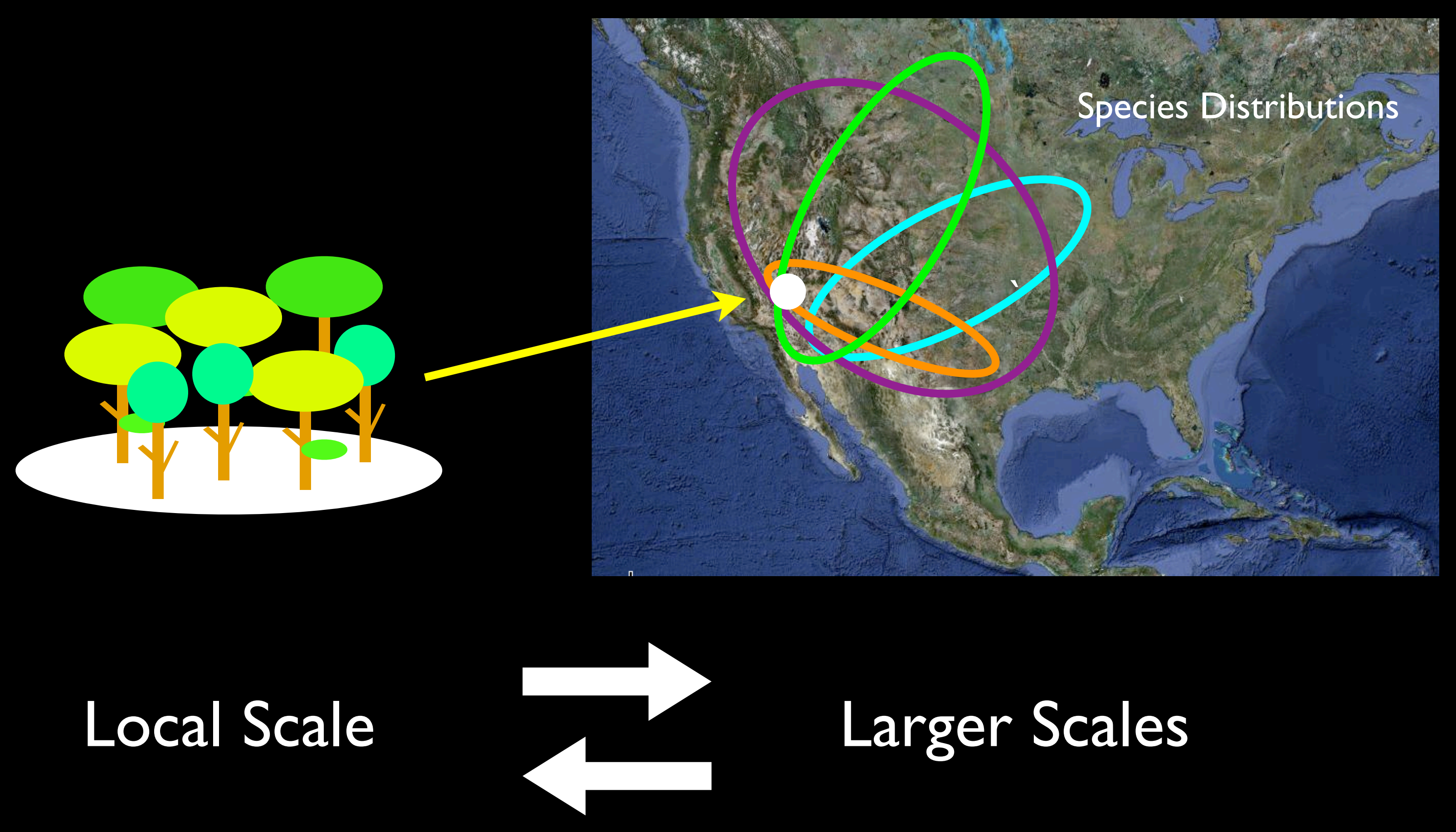




\section{Take Home}

"Pattern is neither a property of the system alone nor of the observer, but an interaction between them." Simon Levin 1989 


\section{Acknowledgements}

Todd Wojtowicz*

Jamie Lamit

Financial Support

NAU IGERT Program

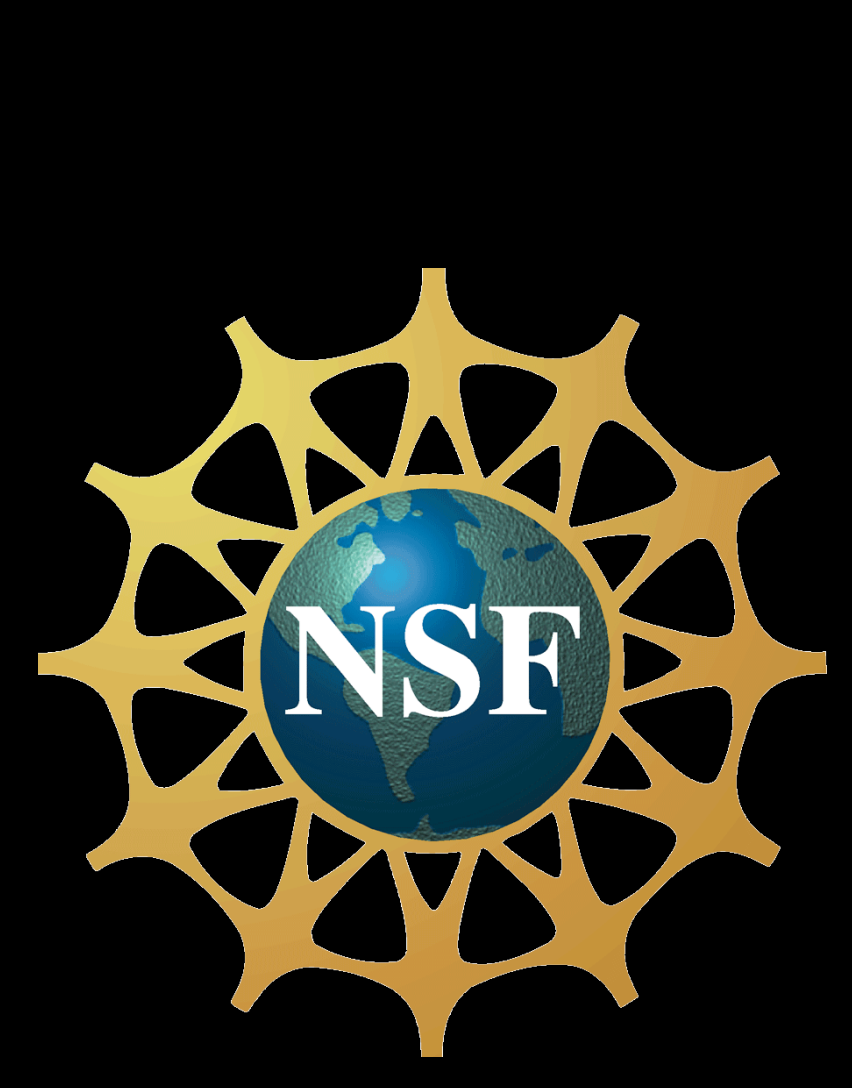

\section{Analytical Software}

\section{EcoSim (Aquired Intelligence and Kesey-Bear, Inc.)}

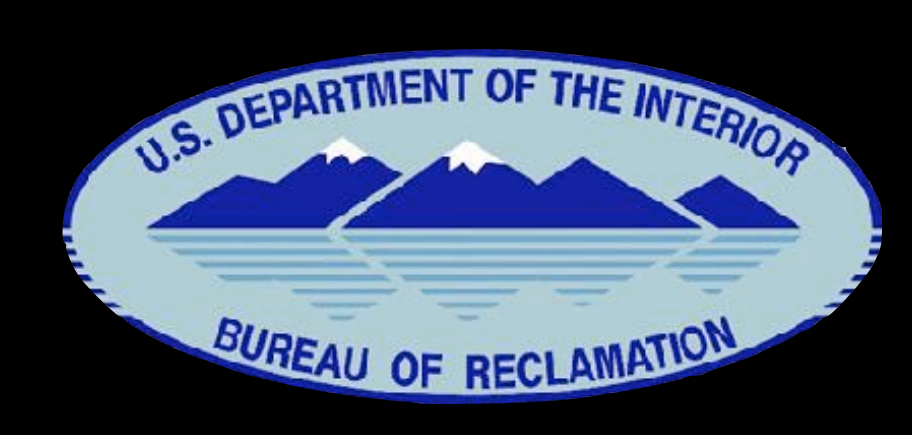

statistical computing language 


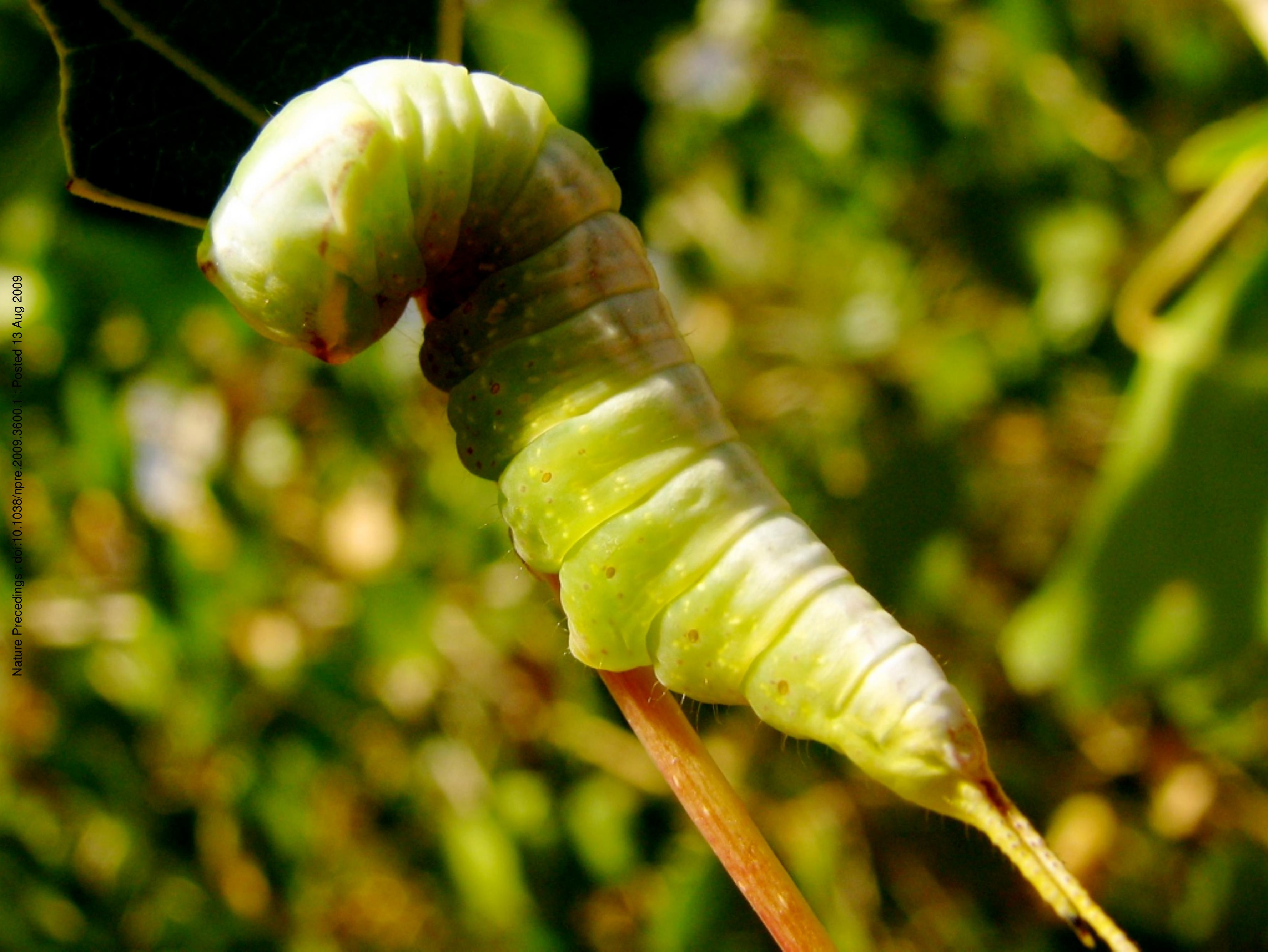

